\title{
Present status of inclusive rare $B$ decays
}

\author{
Tobias Hurth* \\ CERN, Theory Division, CH-1211 Geneva 23, Switzerland, \\ and SLAC, Stanford University, Stanford, California 94309, USA
}

(Published 7 October 2003)

\begin{abstract}
Most inclusive rare $B$ decays are important modes of flavor physics due to the small hadronic uncertainties. In this article, the author gives a status report on such decays, highlighting recent developments and open problems. The focus is on the decay modes $B \rightarrow X_{s, d} \gamma, B \rightarrow X_{s} \ell^{+} \ell^{-}$, and $B \rightarrow X_{s} \nu \bar{\nu}$ and on their role as laboratories in the search for new physics. The experimental data already available from CLEO and the $B$ factories BABAR and BELLE are collected and discussed. The article then reviews the next-to-leading-log (NLL) and next-to-next-to-leading log (NNLL) QCD calculations of the inclusive decay rates that were recently completed and discusses future prospects, especially the issue of the charm-mass-scheme ambiguity. The phenomenological impact of these decay modes, in particular, on the CKM phenomenology and on the indirect search for supersymmetry, is analyzed. Direct $C P$ violation in inclusive rare $B$ decays is briefly treated, as are the rare kaon decays $K^{+} \rightarrow \pi^{+} \nu \bar{\nu}$ and $K_{L} \rightarrow \pi^{0} \nu \bar{\nu}$, which offer complementary, theoretically clean information.
\end{abstract}

\section{CONTENTS}

I. Introduction

II. Strong Interaction in $B$ Decays

III. Experimental Status

A. Experimental data on $B \rightarrow X_{s} \gamma$

B. Photon spectrum of $B \rightarrow X_{s} \gamma$

C. Experimental status of $B \rightarrow X_{s} \ell^{+} \ell^{-}$and $B \rightarrow X_{d} \gamma$

IV. Perturbative Calculations in $B \rightarrow X_{s, d} \gamma$

V. Perturbative Calculations in $B \rightarrow X_{s} \ell^{+} \ell^{-}$and $B \rightarrow X_{s} \bar{\nu} \nu$

VI. Nonperturbative Contributions

A. Inclusive decay rates of $B$ mesons

B. Nonperturbative corrections to $B \rightarrow X_{s, d} \gamma$ and $B \rightarrow X_{s} \ell^{+} \ell^{-}$

VII. Phenomenology

A. Standard-model prediction of $B \rightarrow X_{s} \gamma$

B. CKM phenomenology with $B \rightarrow X_{s, d} \gamma$

C. Role of $b \rightarrow$ s gluon for $B \rightarrow X_{\text {no charm }}$

D. Phenomenology of $B \rightarrow X_{s} \ell^{+} \ell^{-}$

E. Golden mode $B \rightarrow X_{s} \bar{\nu} \nu$

VIII. Indirect Search for Supersymmetry
A. Generalities
B. Constraints from $B \rightarrow X_{s} \gamma$
C. Constraints from $B \rightarrow X_{s} \ell^{+} \ell^{-}$

IX. Direct $C P$ Violation in $b \rightarrow s$ Transitions

X. Further Opportunities

XI. Summary

Acknowledgments

References

\section{INTRODUCTION}

The precise test of the flavor structure and the mechanism of $C P$ violation of the standard model is at the center of today's research in high-energy physics. By

*Electronic address: tobias.hurth@cern.ch and hurth@slac.stanford.edu definition, flavor physics deals with that part of the standard model that distinguishes between the three generations of fundamental fermions. It is still a mystery why there are exactly three generations. The origin of the fermion masses and their mixing is also unknown; in particular, the standard model does not explain the hierarchical pattern of these parameters. Flavor physics can be regarded as the least tested part of the standard model. This is reflected in the rather large error bars of several flavor parameters such as the mixing parameters at the $20 \%$ level (Hagiwara et al., 2002).

However, the experimental situation concerning flavor physics is drastically changing. Several $B$-physics experiments are successfully running at the moment, and, in the coming years, new facilities will start to explore $B$ physics with increasing sensitivity and within various experimental settings. Apart from the CLEO experiment at the Cornell Electron-Positron Storage Ring (Ithaca, NY; CLEO Collaboration, 2003), two $B$ factories, operating at the $Y(4 S)$ resonance in an asymmetric mode, are successfully obtaining data: the BABAR experiment at SLAC (Stanford, CA; BABAR Colloboration, 2003) and the BELLE experiment at KEK (Tsukuba, Japan; BELLE Collaboration, 2003). Besides the hadronic $B$-physics program at Fermilab (Batavia, Illinois; Anikeev et al., 2002), there are $B$-physics experiments planned at other hadronic colliders. All three experiments within the LHC project at CERN in Geneva have strong $B$-physics programs (Ball et al., 2000). An independent $B$-physics experiment, $B \mathrm{TeV}$, is also planned (Wang, 2002) at Fermilab. The main motivation for a $B$-physics program at hadron colliders is the huge $b$-quark production cross section with respect to the one at $e^{+} e^{-}$machines.

While the era of electroweak precision physics focusing on the gauge sector of the standard model draws to a close with the completion of the LEP experiments at CERN and the SLC experiment at Stanford, the era of 

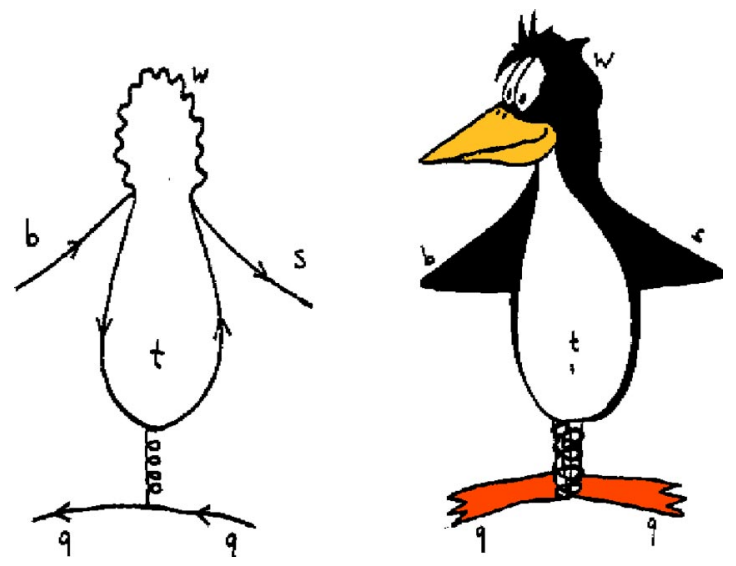

FIG. 1. (Color in online edition) Penguin decays of $B$ mesons (courtesy A. Lenz).

precision flavor physics, focusing on the scalar sector of the standard model, has just begun with the start of the $B$ factories.

The $B$ system represents an ideal framework for the study of flavor physics. Since the $b$-quark mass is much larger than the typical scale of the strong interaction $\Lambda_{Q C D}$, long-distance strong interactions are generally less important and are under better control than in kaon physics, thanks to the expansion in that heavy mass. Moreover, Glashow-Iliopoulos-Maiani suppression is not active in loop diagrams involving the top quarks, which leads to experimentally accessible rare decays and to large $C P$-violating effects within $B$ physics. Thus the $C P$ violation in the $B$ system represents an important independent test of the standard-model description of CP violation (see Hurth et al., 2001; Abe et al., 2002b; Aubert et al., 2002b). B-meson decays also allow for a rich Cabibbo-Kobayashi-Maskawa (CKM) phenomenology as well as stringent tests of the unitarity of the CKM matrix.

The so-called rare decays are of particular interest. These processes represent flavor-changing neutral currents (FCNC's) and occur in the standard model only at the loop level. They also are referred to as "penguin decays" (see Fig. 1), a name first introduced by Ellis et al. (1977) as the result of a bet.

In contrast to the exclusive rare $B$ decay modes, the inclusive ones are theoretically clean observables, because no specific model is needed to describe the hadronic final states. For instance, the decay width $\Gamma(B$ $\left.\rightarrow X_{s} \gamma\right)$ is well approximated by the partonic decay rate $\Gamma\left(b \rightarrow X_{s}^{\text {parton }} \gamma\right)$, which can be analyzed within the framework of renormalization-group-improved perturbation theory. Nonperturbative contributions play only a subordinate role and can be calculated in a modelindependent way by using the heavy-quark expansion.

The role of inclusive rare $B$ decays is twofold: on the one hand, they are relevant to the determination of CKM matrix elements. On the other hand, they are particularly sensitive to new physics beyond the standard model, since additional contributions to the decay rate, in which standard-model particles are replaced by new particles, such as the supersymmetric charginos or gluinos, are not suppressed by additional factors $\alpha /(4 \pi)$ relative to the standard-model contribution. This makes it possible to observe new physics indirectly-a strategy complementary to the direct production of new (supersymmetric) particles. The latter production is reserved for the planned hadronic machines such as the LHC at CERN, while the indirect search of the $B$ factories already implies significant restrictions for the parameter space of supersymmetric models and, thus, leads to important clues for the direct search of supersymmetric particles.

It is even possible that these rare processes will lead to the first evidence of new physics outside the neutrino sector by a significant deviation from the standardmodel prediction, for example, in the observables concerning direct $C P$ violation within the $\Delta F=1$ sector. Such a measurement would definitely not be in conflict with the recent measurements of $C P$ violation in the $B_{d}$ system, which confirms the standard-model predictions at the 10\% level (Abe et al., 2002b; Aubert et al., 2002b). But in the long run, after new physics has already been discovered, inclusive rare $B$ decays will also play an important role in analyzing in greater detail the underlying new dynamics.

The expression inclusive rare $B$ decay is loosely defined and calls for a precise definition. Within the present paper it is understood as a FCNC process $B$ $\rightarrow X Y$, where $B$ denotes a $B^{ \pm}, B_{d}$, or $B_{s}$ meson. $X$ is an inclusive hadronic state containing no charmed particles, and $Y$ is a state built out of leptons, neutrinos, and photons. The possibilities for $Y$ are, for example, $\gamma$ (one particle), $\ell^{+} \ell^{-}, \gamma \gamma$, or $\nu \bar{\nu}$ (two particles), etc. The most interesting ones are $B \rightarrow X_{s, d} \gamma, B \rightarrow X_{s} \ell^{+} \ell^{-}$, and $B$ $\rightarrow X_{s} \nu \bar{\nu}$, on which we shall focus in this paper. Clearly, the cases with $X=\varnothing$ are regarded as exclusive decay modes. Nevertheless, for example, the rare decay $B_{s, d}$ $\rightarrow \ell^{+} \ell^{-}$is also theoretically rather clean, in contrast to other exclusive $B$ rare modes.

In 1993, the first evidence for a rare $B$-meson decay was found by the CLEO Collaboration. At CESR, the exclusive electromagnetic penguin process $B \rightarrow K^{*} \gamma$ was measured (Ammar et al., 1993). Among inclusive rare $B$ decays, the $B \rightarrow X_{s} \gamma$ mode is the most prominent, because it has already been measured by several independent experiments (Alam et al., 1995; Barate et al., 1998; Abe et al., 2001a; Chen et al., 2001; Aubert et al., 2002d, 2002e, 2002f). The stringent bounds obtained from that mode on various nonstandard scenarios (see Borzumati et al., 2000; Degrassi et al., 2000; Besmer et al., 2001; Carena et al., 2001) are also a clear example of the importance of theoretically clean FCNC observables in discriminating among new-physics models. In addition, the inclusive $B \rightarrow X_{s} \ell^{+} \ell^{-}$transition is already accessible at $B$ factories (Kaneko et al., 2003). It represents a new source of theoretically clean observables, complementary to the $B \rightarrow X_{s} \gamma$ rate. In particular, kinematic observables such as the invariant dilepton mass spectrum and the forward-backward asymmetry in $B \rightarrow X_{s} \ell^{+} \ell^{-}$pro- 
vide clean information on short-distance couplings not accessible in $B \rightarrow X_{s} \gamma$ (Ali et al., 1995; Hewett, 1996; Kruger and Sehgal, 1996).

Although the general focus at present within flavor physics is on $B$ systems, kaon physics offers interesting complementary opportunities in the search for new physics, among them the exclusive rare decays $K^{+}$ $\rightarrow \pi^{+} \nu \bar{\nu}$ and $K_{L} \rightarrow \pi^{0} \nu \bar{\nu}$. They are especially interesting in view of the current experiments at Brookhaven National Laboratory (USA) and suggested experiments at Fermilab (USA) and at KEK (Japan). They are also theoretically clean observables.

This review is meant as a status report to highlight recent developments and open problems; for technical tools the reader is often guided to excellent reviews that already exist in the literature. The paper is organized as follows: Section II briefly discusses the role of the strong interaction within flavor physics. In Sec. III the experimental status of rare $B$ decays is summarized. Sections IV and $\mathrm{V}$ focus on the perturbative calculations; the nonperturbative corrections are treated in Sec. VI. Phenomenological implications are discussed in Sec. VII. Section VIII explores the implications of these decays for our search for physics beyond the standard model. In Sec. IX direct $C P$ violation is considered, and in Sec. $\mathrm{X}$ the complementary role of rare kaon decays in precision flavor physics. A summary is presented in Sec. XI.

\section{STRONG INTERACTION IN B DECAYS}

Quark flavor physics is governed by the interplay of strong and weak interactions. One of the main difficulties in examining the observables in flavor physics is the influence of the strong interaction. As is well known, for matrix elements dominated by long-distance strong interactions, there is no adequate quantitative solution available in quantum field theory. The resulting hadronic uncertainties restrict the opportunities in flavor physics significantly, in particular within the indirect search for new physics.

The current discussion of the new $g-2$ muon data (Bennett et al., 2002) also reflects this issue (for a recent review, see de Rafael, 2002): although the hadronic selfenergy contribution to the $g-2$ observable can be determined by experimental data, the results found from $e^{+} e^{-}$-based data and from the $\tau$-based data differ from each other. Furthermore, the well-known light-by-light scattering contribution can only be modeled at present. It is obvious that these hadronic uncertainties make it difficult to deduce strict constraints on a new-physics scenario from this measurement.

There are several fundamental tools available, which are directly based on QCD. High hopes for precise QCD predictions are placed on lattice gauge theoretical calculations. While there are competitive predictions from lattice gauge theory for form factors of semileptonic $B$ decays, pure hadronic decays are less accessible to these methods (Bernard, 2001; Lellouch, 2001, 2002; Lellouch and Luscher, 2001; Sachrajda, 2001).
Another approach is the method of factorization (Bjorken, 1989; Dugan and Grinstein, 1991; Politzer and Wise, 1991). This method has recently been systemized for nonleptonic decays in the heavy-quark limit (Beneke et al., 2000; Bauer et al., 2001; Keum et al., 2001; Keum and Sanda, 2003). However, within this approach, a quantitative method for estimating the $1 / m_{b}$ corrections to this limit is missing (Beneke, Buchalla, et al., 2001; Ciuchini et al., 2001). A promising step in this direction was recently presented by Bauer et al. (2001) and Beneke et al. (2002).

Further well-known fundamental methods whose applications and precision are also somewhat restricted are chiral perturbation theory (Gasser, 2000; Colangelo and Isidori, 2001), heavy-quark effective theory (Isgur and Wise, 1989, 1990; Neubert, 1994b), QCD sum rules (Braun, 1998; Khodjamirian and Ruckl, 1998; Shifman, 1998; Colangelo and Khodjamirian, 2000), and the $1 / N$ expansion (Manohar, 1998; de Rafael, 2001).

In view of this, the goal must be to minimize theoretical uncertainties with the help of an optimized combination of different fundamental methods solely based on QCD. This can only be done for a selected number of observables in flavor physics. It is also clear that an active cooperation between theory and experiment is necessary in order to make progress on this issue.

There are a few golden channels in which the hadronic physics can be disentangled and clean tests of the standard model are possible. Moreover, there are also observables, dominated by perturbative contributions, which make precision flavor physics possible in the near future. Among them, inclusive rare $B$ decays play the most important role. Inclusive decay modes are theoretically clean and represent a theoretical laboratory of perturbative QCD. In particular, the decay width $\Gamma(B$ $\left.\rightarrow X_{s} \gamma\right)$ is well approximated by the partonic decay rate $\Gamma\left(b \rightarrow X_{s}^{\text {parton }} \gamma\right)$, which can be analyzed in renormalization-group-improved perturbation theory as

$$
\Gamma\left(B \rightarrow X_{s} \gamma\right)=\Gamma\left(b \rightarrow X_{s}^{\text {parton }} \gamma\right)+\Delta^{\text {nonpert }} .
$$

Nonperturbative effects, $\Delta^{\text {nonpert }}$, play a subordinate role and are under control, thanks to the heavy-mass expansion (Chay et al., 1990; Bigi et al., 1992, 1997; Manohar and Wise, 2000) and the assumption of quarkhadron duality (Shifman, 2000; Bigi and Uraltsev, 2001).

Thus, in general, inclusive decay modes should be preferred to exclusive ones from the theoretical point of view. The inclusive modes $B \rightarrow X_{s(d)} \gamma$ and $B$ $\rightarrow X_{s(d)} \ell^{+} \ell^{-}$can be measured by the electron-positron experiments ( $B$ factories, CLEO) with their kinematic constraints and their controlled backgrounds, while they are more difficult to measure at hadronic machines. Exclusive decay modes are more accessible to experiments, in particular, at hadronic machines, but, in contrast to the inclusive modes, they have in general large nonperturbative QCD contributions, which make it difficult to deduce valuable information on new physics. However, as mentioned in the Introduction, the exclusive decays $B_{d, s} \rightarrow \mu^{+} \mu^{-}$are distinguished observables at hadronic colliders. 


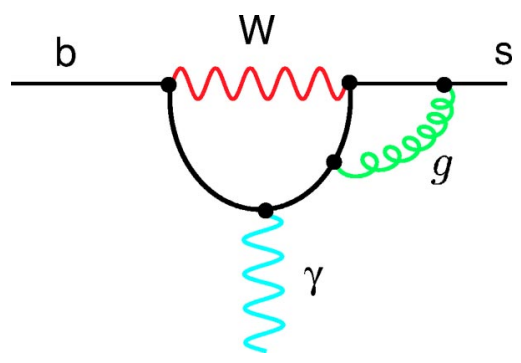

FIG. 2. (Color in online edition) QCD corrections to the decay $B \rightarrow X_{s} \gamma$.

Within inclusive $B$ decay modes, short-distance QCD effects turn out to be very important. For example, in the decay $B \rightarrow X_{s} \gamma$ they lead to a tremendous rate enhancement. These effects are induced by hard-gluon exchange between the quark lines of the one-loop electroweak diagrams (Fig. 2).

The QCD radiative corrections bring in large logarithms of the form $\alpha_{s}^{n}\left(m_{b}\right) \ln ^{m}\left(m_{b} / M\right)$, where $M=m_{t}$ or $M=m_{W}$ and $m \leqslant n$ (with $n=0,1,2, \ldots$ ). This is a natural feature of any process in which two different mass scales are present. In order to get a reasonable result at all, one has to resum at least the leading-log (LL) series

$$
\alpha_{s}^{n}\left(m_{b}\right) \ln ^{n}\left(m_{b} / M\right) \quad(\mathrm{LL})
$$

with the help of renormalization-group techniques. Working to next-to-leading-log (NLL) precision means that one is also resumming all the terms of the form

$$
\alpha_{s}\left(m_{b}\right) \alpha_{s}^{n}\left(m_{b}\right) \ln ^{n}\left(m_{b} / M\right) \text { (NLL). }
$$

A suitable framework in which to achieve the necessary resummations of the large logs is an effective lowenergy theory with five quarks, obtained by integrating out the heavy particles, which, in the standard model, are the electroweak bosons and the top quark. The standard method of the operator-product expansion allows for a separation of the meson decay amplitude into two distinct parts, the long-distance contributions contained in the operator matrix elements and the short-distance physics described by the so-called Wilson coefficients (see Fig. 3). In the case of $B$ decays, the $W$ boson and the top quark with mass larger than the factorization scale are integrated out, that is, removed from the theory as dynamical fields. The effective interaction Hamiltonian can be written

$$
H_{\text {eff }}=-\frac{4 G_{F}}{\sqrt{2}} \sum C_{i}\left(\mu, M_{\text {heavy }}\right) F_{i}^{C K M} \mathcal{O}_{i}(\mu),
$$

where $\mathcal{O}_{i}(\mu)$ are the relevant operators, $C_{i}\left(\mu, M_{\text {heavy }}\right)$ are the corresponding Wilson coefficients, and $F_{i}^{C K M}$ the

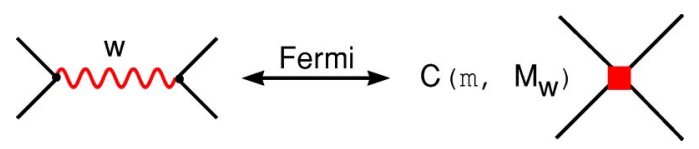

FIG. 3. (Color in online edition) Operator-product expansion: full vs effective theory. relevant CKM factors. As the heavy fields are integrated out, the complete top and $W$ mass dependence are contained in the Wilson coefficients. Working out a convenient set of quantities, both in the effective (low-energy) theory and in the full (standard-model) theory, and requiring equality (matching) up to terms suppressed by higher powers of $m_{W}$ or $m_{t}$, these coefficients can be determined. At the high scale $\mu_{W} \approx m_{W}, m_{t}$, the matrix elements of the operators in the effective theory lead to the same logarithms as the full theory calculation. Consequently, the Wilson coefficients $C_{i}\left(\mu_{W}\right)$ only pick up small QCD corrections, which can be calculated in fixedorder perturbation theory.

Within this framework QCD corrections for the decay rates are twofold: the ingredients are the order- $\alpha_{s}$ corrections to the matrix elements of the various operators and the order- $\alpha_{s}$ corrections to the Wilson coefficients, of course both at the low-energy scale $\mu_{b} \approx m_{b}$. Only the sum of the two contributions is independent of renormalization scheme and scale; in fact, from the $\mu$ independence of the effective Hamiltonian, one can derive a renormalization-group equation for the Wilson coefficients $C_{i}(\mu)$ :

$$
\mu \frac{d}{d \mu} C_{i}(\mu)=\gamma_{j i} C_{j}(\mu)
$$

where the matrix $\gamma$ is the anomalous-dimension matrix of the operators $\mathcal{O}_{i}$, which describes the anomalous scaling of the operators with respect to the one at the classical level. At leading order, the solution is given by

$$
\begin{aligned}
\widetilde{C}_{i}(\mu) & =\left[\frac{\alpha_{s}\left(\mu_{W}\right)}{\alpha_{s}(\mu)}\right]^{\tilde{\gamma}_{i i}^{(0)} / 2 \beta_{0}} \widetilde{C}_{i}\left(\mu_{W}\right) \\
& =\left[\frac{1}{1+\beta_{0} \frac{\alpha_{s}(\mu)}{4 \pi} \ln \frac{\mu_{W}^{2}}{\mu^{2}}}\right]^{\tilde{\gamma}_{i i}^{(0)} / 2 \beta_{0}} \widetilde{C}_{i}\left(\mu_{W}\right)
\end{aligned}
$$

with $\mu d / d \mu \alpha_{s}=-2 \beta_{0} \alpha_{s}^{2} /(4 \pi) ; \beta_{0}$ and $\widetilde{\gamma}_{i i}^{0}$ correspond to the leading anomalous dimension of the coupling constant and the operators, respectively. The tilde indicates that the diagonalized anomalous-dimension matrix is used. Equation (2.6) to LL precision makes the renormalization-group improvement transparent. It represents a summation of the form of Eq. (2.2).

There are three principal calculational steps leading to the leading-log (next-to-leading-log) result within the effective-field theory approach, for a pedagogical review see Buras (1998):

- Step 1: The full standard-model theory has to be matched with the effective-field theory at the scale $\mu$ $=\mu_{W}$, where $\mu_{W}$ denotes a scale of order $m_{W}$ or $m_{t}$. As mentioned above, the Wilson coefficients $C_{i}\left(\mu_{W}\right)$ only pick up small QCD corrections, which can be calculated in fixed-order perturbation theory. In the LL (NLL) program, the matching has to be worked out at the $O\left(\alpha_{s}^{0}\right)\left[O\left(\alpha_{s}^{1}\right)\right]$ level.

- Step 2: The evolution of these Wilson coefficients from $\mu=\mu_{W}$ down to $\mu=\mu_{b}$ has to be performed with 


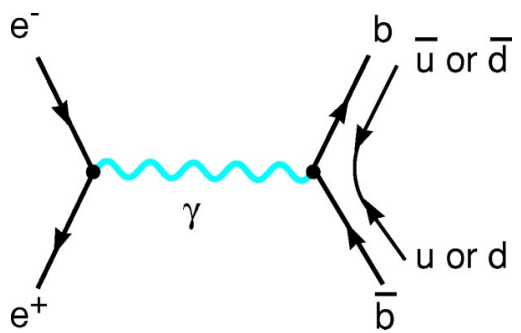

FIG. 4. (Color in online edition) $e^{+} e^{-} \rightarrow \mathrm{Y}(4 S)$ $\rightarrow B^{+} B^{-}, B^{0} \bar{B}^{0}$.

the help of the renormalization group, where $\mu_{b}$ is of the order of $m_{b}$. Since the matrix elements of the operators evaluated at the low scale $\mu_{b}$ are free of large logarithms, the latter are contained in resummed form in the Wilson coefficients. For a LL (NLL) calculation, this renormalization-group step has to be done using the anomalous-dimension matrix up to or$\operatorname{der} \alpha_{s}^{1}\left(\alpha_{s}^{2}\right)$.

- Step 3: For LL (NLL) precision, the corrections to the matrix elements of the operators $\langle s \gamma|\mathcal{O} i(\mu)| b\rangle$ at the scale $\mu=\mu_{b}$ have to be calculated to order- $\alpha_{s}^{0}\left(-\alpha_{s}^{1}\right)$ precision. This also includes bremsstrahlung corrections.

Finally, we stress that the step from the leading (LL) to the next-to-leading (NLL) order within the framework of the renormalization-group-improved perturbation theory is not only a quantitative one, increasing the precision of the theoretical prediction, but also a qualitative one, which tests the validity of the perturbative approach in the given problem.

\section{EXPERIMENTAL STATUS}

\section{A. Experimental data on $B \rightarrow X_{s} \gamma$}

Among inclusive rare $B$ decays, the $B \rightarrow X_{s} \gamma$ mode is the most prominent because it has already been measured by several independent $e^{+} e^{-}$experiments (see Fig. 4), mostly at the $Y(4 S)$ resonance (Alam et al., 1995; Barate et al., 1998; Abe et al., 2001a; Chen et al., 2001; Aubert et al., 2002d, 2002f; see also Lingel et al., 1998; Stone, 2001; Thorndike, 2002). In 1993, the first evidence for a penguin-induced $B$-meson decay was found by the CLEO Collaboration. At CESR, they measured the exclusive electromagnetic penguin process $B$ $\rightarrow K^{*} \gamma$ (Ammar et al., 1993). The inclusive analog $B$ $\rightarrow X_{s} \gamma$, which is the quantity of theoretical interest, was also found by the CLEO Collaboration through the measurement of its characteristic photon energy spectrum in 1994. Since this process is dominated by the twobody decay $b \rightarrow s \gamma$, its photon energy spectrum is expected to be a smeared delta function centered at $E_{\gamma}$ $\approx m_{b} / 2$, where the smearing is due to perturbative gluon bremsstrahlung and to the nonperturbative motion of the $b$ quark within the $B$ meson.
Only the high part of the $B \rightarrow X_{s} \gamma$ photon spectrum is observed. Some lower cutoff in the photon energy was imposed in order to suppress the background from other $B$-decay processes. The $B \bar{B}$ background mainly arises from the processes $B \rightarrow \pi^{0} X$ and $\pi^{0} \rightarrow \gamma_{1} \gamma_{2}$ or $B \rightarrow \eta X$ and $\eta \rightarrow \gamma_{1} \gamma_{2}$, where $\gamma_{1}$ has high energy and $\gamma_{2}$ either has energy too low to be observed or is not in the geometric acceptance of the detector. Moreover, there is a small component $(\sim 5 \%)$ from the process $B \rightarrow \bar{n} X$ or $B \rightarrow K_{L} X$, in which the antineutron or the neutral kaon interacts hadronically with the electromagnetic calorimeter, faking a photon.

Therefore only the "kinematic" branching ratio for $B \rightarrow X_{s} \gamma$ in the range between $E_{\gamma}=2.2 \mathrm{GeV}$ and the kinematic end point at $E_{\gamma}=2.7 \mathrm{GeV}$ could be measured directly within this first CLEO measurement. To obtain from this measurement the "total" branching ratio, one has to know the fraction $R$ of the $B \rightarrow X_{s} \gamma$ events with $E_{\gamma} \geqslant 2.2 \mathrm{GeV}$. This was first found by Ali and Greub (1991, 1995), who took the motion of the $b$ quark in the $B$ meson into account by using a phenomenological model (Ali and Pietarinen, 1979; Altarelli et al., 1982) and by incorporating a large systematic error for this model dependence. Using this theoretical input regarding the photon energy spectrum, the CLEO Collaboration used the value $R=0.87 \pm 0.06$, leading to the CLEO branching ratio (Alam et al., 1995)

$$
\mathcal{B}\left(B \rightarrow X_{s} \gamma\right)=\left(2.32 \pm 0.57_{\text {stat }} \pm 0.35_{\text {syst }}\right) \times 10^{-4} \text {. }
$$

The first error is statistical and the second is systematic (including model dependence). This measurement was based on a sample of $2.2 \times 10^{6} B \bar{B}$ events.

Besides the high-energy cutoff to suppress the background from other $B$ decays, two different techniques were used to suppress the continuum background in this first CLEO measurement. In the first (semi-inclusive) technique, all products were reconstructed as in the exclusive measurement. The background in the measurement of exclusive modes is naturally low because of kinematical constraints and the beam energy constraint. In order to reduce the combinatoric background, only $K(n \pi) \gamma$, with $n \leqslant 4$ and at most one $\pi^{0}$, were chosen as final states in this analysis, which accounts for $\sim 50 \%$ of the inclusive rate. In the second (fully inclusive) technique, only the photon was explicitly reconstructed. As shown in Fig. 5, there are very large backgrounds, both from the initial-state radiation (ISR) process $e^{+} e^{-}$ $\rightarrow q \bar{q} \gamma$, where one of the beam electrons radiates a hard photon before annihilation, and from inclusive $\pi^{0} / \eta$ production in which one of the photons from the decay is not detected. Background suppression was therefore more difficult with this technique. For this purpose, topological differences between the spherical $B \bar{B}$ events and the two jets $e^{+} e^{-} \rightarrow q \bar{q}$ as shown in Fig. 6 were used. While the signal events are spherical because the $B$ mesons are almost at rest at the $Y(4 S)$ resonance, the continuum events have a jetlike structure. With the help of a neural network, several event-shape variables were combined into a single one, which tends towards +1 for $b$ 


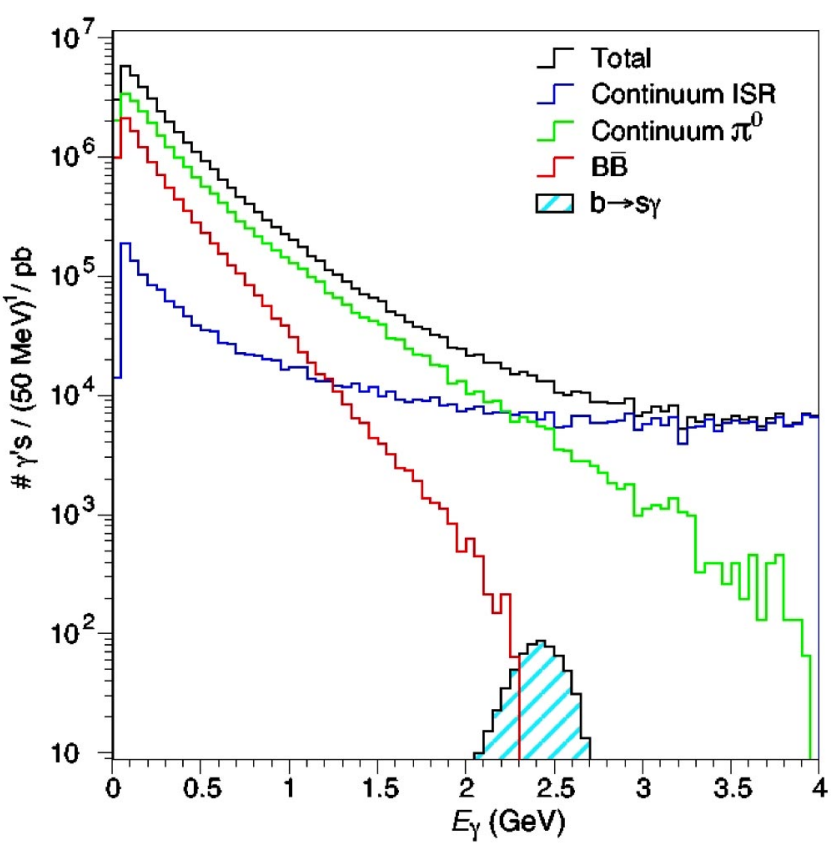

FIG. 5. (Color in online edition) Levels of inclusive photons from various background processes at $\Upsilon(4 S)$ and the expected signal from $b \rightarrow s \gamma$ : ISR, $B \bar{B}$, and $\pi^{0}$ backgrounds (from the bottom to the top at $E_{\gamma}=0.5$ ). From Thorndike, 2002.

$\rightarrow s \gamma$ and towards -1 for the ISR and $q \bar{q}$ processes; the signal was extracted from a one-parameter fit to that variable.

The signal efficiency $(32 \%)$ was very high with respect to the first technique (9\%). However, the first technique has a better signal-to-noise ratio, so that the two methods had nearly equal sensitivity. In the first CLEO measurement in 1994, they found $\mathcal{B}\left(B \rightarrow X_{s} \gamma\right)=(2.75$ $\left.\pm 0.67_{\text {stat }}\right) \times 10^{-4}$ using the first technique and $\mathcal{B}(B$ $\left.\rightarrow X_{s} \gamma\right)=\left(1.88 \pm 0.74_{\text {stat }}\right) \times 10^{-4}$ using the second technique. The branching ratio stated above in Eq. (3.1) represents the average of the two measurements, taking into account the correlation between the two techniques.

In 1999, CLEO presented a preliminary improved measurement (Ahmed et al., 1999), which was based on $53 \%$ more data $\left(3.3 \times 10^{6}\right.$ events). They also used the slightly wider $E_{\gamma}$ window starting at $2.1 \mathrm{GeV}$. The relative error dropped by a factor of almost $\sqrt{3}$. In 2002, CLEO published a new measurement (Chen et al., 2001) based on three times more data $\left(10 \times 10^{6}\right.$ events $)$. The spectrum down to $2.0 \mathrm{GeV}$ was used, which included almost $90 \%$ of the $B \rightarrow X_{s} \gamma$ yield. This also led to a sig-
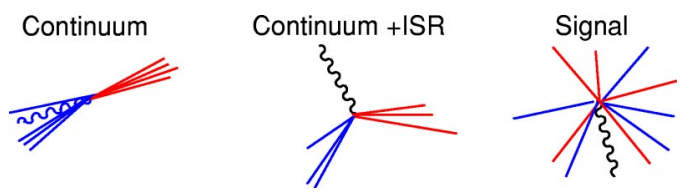

FIG. 6. (Color in online edition) Examples of idealized event shapes. The straight lines indicate hadrons and the wavy lines photons. From Stone, 2001. nificant background from $B$ decay processes other than $B \rightarrow X_{s} \gamma$, located within $2.0-2.2 \mathrm{GeV}$. This $B \bar{B}$ background arose from two components. The first was inclusive $\pi^{0} / \eta$ decays, which accounted for $\sim 90 \%$ of the background. This was estimated by Monte Carlo in which the inclusive $\pi^{0} / \eta$ spectra were tuned with independent processes to replicate the data. Second, hadronic interactions of antineutrons and neutral kaons in the electromagnetic calorimeter may have faked a photon candidate. However, their lateral profile was different from that of real photons, which allowed a background subtraction. The continuum background was suppressed with the same two approaches as in the first measurement, but within a fully integrated analysis. What remained of the continuum background was subtracted using off-resonance data.

In order to obtain the corrected branching ratio of $B$ $\rightarrow X_{s} \gamma$, two extrapolations were necessary. What was directly measured was the branching fraction for $B$ $\rightarrow X_{s} \gamma$ plus $B \rightarrow X_{d} \gamma$. The $B \rightarrow X_{d} \gamma$ part was subtracted by using the theory input that, according to the standard-model expectation, the $B \rightarrow X_{d} \gamma$ and the $B$ $\rightarrow X_{s} \gamma$ branching fractions are in the ratio $\left|V_{t d} / V_{t s}\right|^{2}$. Therefore the branching ratio was corrected down by $(4.0 \pm 1.6) \%$ of itself-assuming the validity of the standard-model suppression factor $\left|V_{t d} / V_{t s}\right|^{2}$. Moreover, one had to know again the fraction $R$ of the $B$ $\rightarrow X_{s} \gamma$ events with $E_{\gamma} \geqslant 2.0 \mathrm{GeV}$. In this measurement, the corresponding fraction was estimated to be $R$ $=0.915_{-0.055}^{+0.027}$ using the model of Kagan and Neubert (1999) (see also Sec. III.B), which allowed for the extrapolation of the measured branching ratio to the "total" $B \rightarrow X_{s} \gamma$ branching ratio $\left(E_{\gamma}>0.25 \mathrm{GeV}\right)$. With these two theoretical corrections, the present CLEO measurement for the $B \rightarrow X_{s} \gamma$ branching ratio is

$\mathcal{B}\left(B \rightarrow X_{s} \gamma\right)=\left(3.21 \pm 0.43_{\text {stat }} \pm 0.27_{\text {syst }-0.10_{\text {mod }}}^{+0.18}\right) \times 10^{-4}$.

The errors represent statistics, systematics, and model dependence (due to the extrapolation below $E_{\gamma}$ $=2.0 \mathrm{GeV}$ ), respectively.

There are also data at the $Z^{0}$ peak from the LEP experiments. The ALEPH Collaboration (Barate et al., 1998) has measured the inclusive branching ratio based on $0.8 \times 10^{6} b \bar{b}$ pairs. Their result reads

$\mathcal{B}\left(H_{b} \rightarrow X_{s} \gamma\right)=\left(3.11 \pm 0.80_{\text {stat }} \pm 0.72_{\text {syst }}\right) \times 10^{-4}$.

The signal was isolated in lifetime-tagged $b \bar{b}$ events by the presence of a hard photon associated with a system of high-momentum and high-rapidity hadrons. It should be noted that the branching ratio in Eq. (3.3) involves a weighted average of the $B$ mesons and $\Lambda_{b}$ baryons produced in $Z^{0}$ decays (hence the symbol $H_{b}$ ) different from the corresponding one given by CLEO, which was measured at the $\Upsilon(4 S)$ resonance. High luminosity is more difficult to obtain at higher $e^{+} e^{-}$collision energies. Thus $B \bar{B}$ samples obtained by the LEP experiments are rather small. The rate measured by ALEPH is 
consistent with the CLEO measurement, with an error twice as large as the present CLEO measurement.

BELLE has also presented a measurement (Abe et al., 2001a) based on $6.07 \times 10^{-6} B \bar{B}$ events at the $\Upsilon(4 S)$ resonance. A semi-inclusive analysis was used to reconstruct the $B \rightarrow X_{s} \gamma$ decay from a primary photon, a kaon, and multiple pions $[K(n \pi) \gamma$, with $n \leqslant 4$ and no more than one $\pi^{0}$ ].

The background reduction includes an effective $E_{\gamma}$ $>2.24 \mathrm{GeV}$ photon energy cutoff, which corresponds to a cut in the hadronic mass spectrum of $M_{X_{s}}$ $=2.05 \mathrm{GeV}$ as quoted by Abe et al. (2001a); $E_{\gamma}=\left(M_{B}^{2}\right.$ $\left.-M_{X_{s}}^{2}\right) /\left(2 M_{B}\right)$ :

$\mathcal{B}\left(B \rightarrow X_{s} \gamma\right)=\left(3.37 \pm 0.53_{\text {stat }} \pm 0.42_{\text {syst }} \pm 0.54_{\text {mod }}\right) \times 10^{-4}$,

which is consistent with previous measurements.

BABAR presented two preliminary analyses on the $B \rightarrow X_{s} \gamma$ branching ratio, a fully inclusive and a semiinclusive one (Aubert et al., 2002d, 2002f). The fully inclusive BABAR measurement has used the largest number of $B$ mesons so far. It is based on almost $60 \times 10^{6} B \bar{B}$ events at the $Y(4 S)$ resonance. The method of extracting the signal from the data is similar to what was done for previous measurements: the continuum background was subtracted with the help of off-resonance data. The $B \bar{B}$ contribution was deduced from Monte Carlo predictions.

The high statistics available in this BABAR measurement allowed for additional techniques: a lepton tag on a high-momentum electron or muon was also required to suppress continuum backgrounds. For the $B \rightarrow X_{s} \gamma$ signal events, the lepton arises from the semileptonic decay of the other $B$ meson. Leptons also occur in the continuum background, most notably from the semileptonic decays of charm hadrons, but their production is significantly less frequent and their momentum lower than those from a $B$ decay. Because a lepton tag is imposed on the other $B$ meson, not on the signal $B$, one can reject the continuum background without introducing any model dependence because one does not impose any requirements on the signal decay. A $\times 1200$ reduction of the background was achieved by $5 \%$ efficiency of the lepton tag. This effective method for suppressing the continuum background was possible because of the high statistics of the new BABAR measurement.

The systematic precision was limited by the size of the $B \bar{B}$ background control samples scaling in proportion to the signal sample. It limited the lower bound to $E_{\gamma}$ $>2.1 \mathrm{GeV}$ (measured in the $e^{+} e^{-}$center-of-mass system). The preliminary BABAR measurement is

$\mathcal{B}\left(B \rightarrow X_{s} \gamma\right)=\left(3.88 \pm 0.36_{\text {stat }} \pm 0.37_{\text {syst }}+0.23_{\text {mod }}+0.43^{-4}\right.$.

Besides this fully inclusive analysis, BABAR also presented a preliminary semi-inclusive analysis (Aubert et al., 2002e). Twelve exclusive $b \rightarrow s \gamma$ decays were fully reconstructed, which led to the following measurement of the inclusive branching ratio:

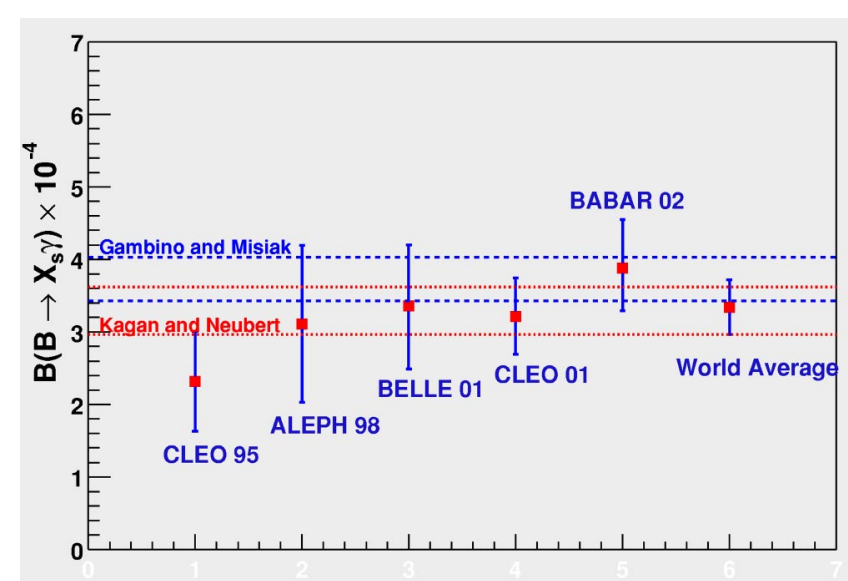

FIG. 7. (Color in online edition) $B \rightarrow X_{s} \gamma$ measurements vs theoretical predictions (see Sec. VII.A). From Aubert et al., 2002d.

$$
\mathcal{B}\left(B \rightarrow X_{s} \gamma\right)=\left(4.4 \pm 0.5_{\text {stat }} \pm 0.8_{\text {syst }} \pm 1.3_{\text {mod }}\right) \times 10^{-4}
$$

The error is much larger than that of the previous semi-inclusive measurements. It includes fewer final states; only states including 1-3 pions rather than 1-4 pions were reconstructed. There is also a large systematic error due to the Monte Carlo modeling of the $X_{s}$ fragmentation into $K n \pi\left(\pi^{0}\right)$, used to calculate the efficiency. The semi-inclusive analysis is based on almost $23 \times 10^{6} B \bar{B}$ events at the $Y(4 S)$ resonance.

When more statistics become available, the fully inclusive strategy using the lepton tag will get priority in the future measurements of the $B \rightarrow X_{s} \gamma$ branching ratio because model dependence and systematic errors can be reduced significantly compared to the semi-inclusive method.

As Fig. 7 shows, all the measurements of the "total" $B \rightarrow X_{s} \gamma$ branching ratio available so far are consistent with each other and with the standard-model predictions (see Sec. VII.A). A weighted average of the available experimental measurements is problematic, because the model-dependence errors (and also the systematic errors) are correlated and differ within the various measurements. A recent analysis taking into account the correlations leads to a world average (Jessop, 2002)

$$
\mathcal{B}\left(B \rightarrow X_{s} \gamma\right)=(3.34 \pm 0.38) \times 10^{-4} \text {. }
$$

With the expected high luminosity of the $B$ factories, the systematic uncertainty in the $B \bar{B}$ background will be reduced along with statistical uncertainties. This reduction in the systematic uncertainty will also allow for a lower photon energy cutoff, which will further reduce the model dependence from the theory-based interpolation to the whole energy spectrum (Aubert et al., 2002d). Thus in the future the lower-energy cutoff in a fully inclusive analysis has to balance the systematic error due to the $B \bar{B}$ background and the model dependence due to the extrapolation. An experimental accuracy below $10 \%$ in the inclusive $B \rightarrow X_{s} \gamma$ mode is possible in the near future. 


\section{B. Photon spectrum of $B \rightarrow X_{s} \gamma$}

The uncertainty regarding the fraction $R$ of the $B$ $\rightarrow X_{s} \gamma$ events above the chosen lower photon energy cutoff $E_{\gamma} \mathrm{GeV}$ quoted in the experimental measurement, also cited as model dependence, should be regarded as a purely theoretical uncertainty: in contrast to the "total" branching ratio of $B \rightarrow X_{s} \gamma$, the photon energy spectrum cannot be calculated directly using the heavy-mass expansion. This is because the operatorproduct expansion breaks down in the high-energy part of the spectrum, where $E_{\gamma} \approx m_{b} / 2$. Therefore the fraction $R$ was calculated by Ali and Greub (1991, 1995) using a phenomenological model (Ali and Pietarinen, 1979; Altarelli et al., 1982), in which the motion of the $b$ quark in the $B$ meson is characterized by two parameters, the average momentum $p_{F}$ of the $b$ quark and the average mass $m_{q}$ of the spectator quark.

The error in the fraction $R$ is essentially obtained by varying the model parameters $p_{F}$ and $m_{q}$ in the range for which the model correctly describes the energy spectrum of the charged lepton in the semileptonic decays $B \rightarrow X_{c} \ell \nu$ and $B \rightarrow X_{u} \ell \nu$, measured by CLEO and ARGUS. Ali and Greub $(1991,1995)$ presented a first comparison between the calculated photon energy spectrum and the one measured by the CLEO Collaboration. The (normalized) measured photon energy spectrum and the theoretical one were in agreement for those values of $p_{F}$ and $m_{q}$ that correctly describe the inclusive semileptonic CLEO data on $B \rightarrow X_{c} \ell \nu$ and $B$ $\rightarrow X_{u} \ell \nu$.

Besides this phenomenological model, more fundamental theoretical methods are available today to implement the bound-state effects, namely, by making use of operator-product expansion techniques in the framework of the heavy-quark effective theory. An analysis along these lines was presented in Kagan and Neubert (1999). As mentioned above, the operator-product expansion breaks down near the end point of the photon energy spectrum; therefore an infinite number of leading-twist corrections have to be resummed into a nonperturbative universal shape function, which determines the light-cone momentum distribution of the $b$ quark in the $B$ meson (Bigi et al., 1993, 1994; Mannel and Neubert, 1994; Manohar and Wise, 1994; Neubert, 1994a; Aglietti and Ricciardi, 2000; Aglietti et al., 2002). The physical decay distributions are then obtained from a convolution of parton model spectra with this shape function. At present this function cannot be calculated, but there is at least some information on the moments of the shape function, which are related to the forward matrix elements of local operators. Ansätze, constrained by the latter information, are used for the shape function. In contrast to the older analysis based on the phenomenological model proposed by Ali and Pietarinen (1979) and Altarelli et al. (1982), the analysis of Kagan and Neubert (1999) includes the full NLL information.

In the latest CLEO measurement (Chen et al., 2001), the phenomenological spectator model (Ali and Pietarinen, 1979; Altarelli et al., 1982; Ali and Greub, 1991,

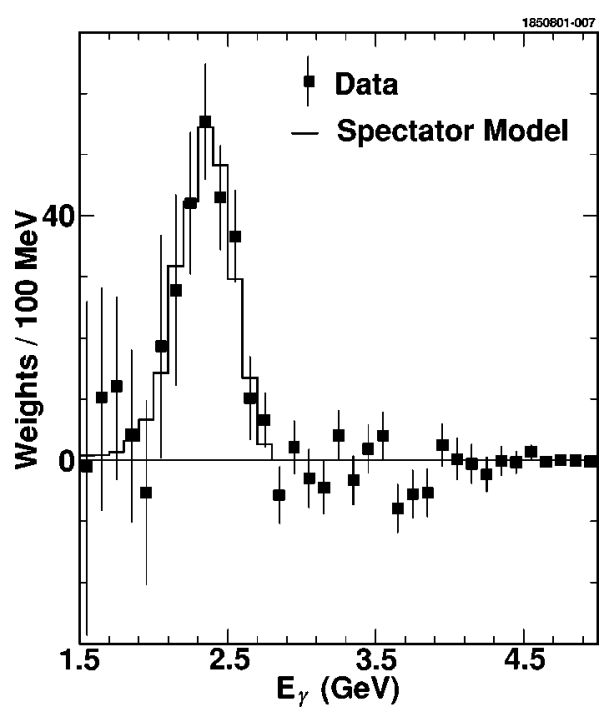

FIG. 8. Photon energy spectrum measured by CLEO and spectrum from Monte Carlo simulation of the spectator model with parameters $\hat{m}_{b}=4.690 \mathrm{GeV}, p_{F}=410 \mathrm{MeV} / c$, a good fit to the data. From Chen et al., 2001.

1995) was used first. The momentum parameter $p_{F}$ and the $b$-quark average mass $\hat{m}_{b}$ were treated as free parameters, which allowed the mean and the width of the photon energy spectrum to be varied (see Fig. 8). The Kagan-Neubert approach was also used by CLEO: the simple two-parameter shape function was fitted to the measured photon spectrum and results very similar to those obtained using the spectator model were seen.

An important observation is that the shape of the photon spectrum is practically insensitive to physics beyond the standard model. As can be seen in Fig. 9, all different contributions to the spectrum (corresponding to the interference terms of the various operators involved; see Sec. IV) have a very similar shape besides the small 8-8 contribution. This implies that we do not have to assume the correctness of the standard model in the experimental analysis.

A precise measurement of the photon spectrum allows us to determine the parameters of the shape function. The latter information is an important input for the determination of the CKM matrix element $V_{u b}$. One takes advantage of the universality of the shape function to lowest order in $\Lambda_{Q C D} / m_{b}$. The same shape function occurs in the description of nonperturbative effects in the end-point region of the $B \rightarrow X_{s} \gamma$ photon spectrum as well as of the $B \rightarrow X_{u} \ell \nu$ charged-lepton spectrum up to higher 1/m$m_{b}$ corrections (Bigi et al., 1993, 1994; Mannel and Neubert, 1994; Manohar and Wise, 1994; Neubert, 1994a; Aglietti and Ricciardi, 2000; Aglietti et al., 2002). Thus from the photon spectrum one can determine the shape function; with the help of the latter and the measurement of the charged-lepton spectrum of $B \rightarrow X_{u} \ell \nu$, one can extract a value for $V_{u b}$. This method represents one of the best ways to measure the CKM matrix element $V_{u b}$. Following this strategy, CLEO has presented the following measurement (Bornheim et al., 2002):

$$
V_{u b}=\left(4.08 \pm 0.56_{\exp } \pm 0.29_{t h}\right) .
$$



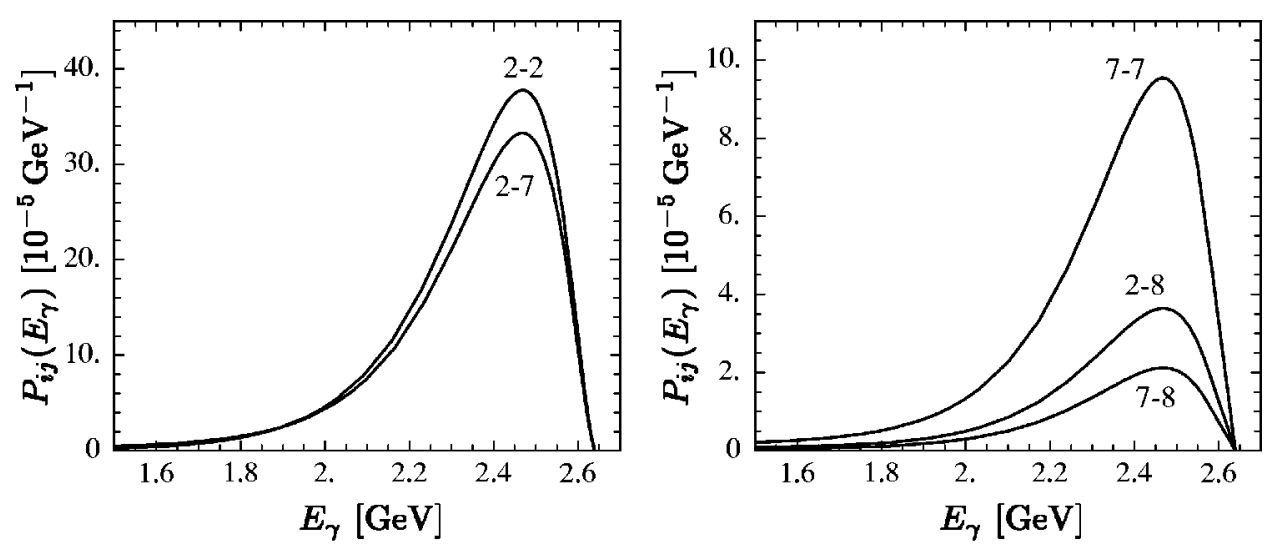

FIG. 9. Different components of the photon spectrum in the
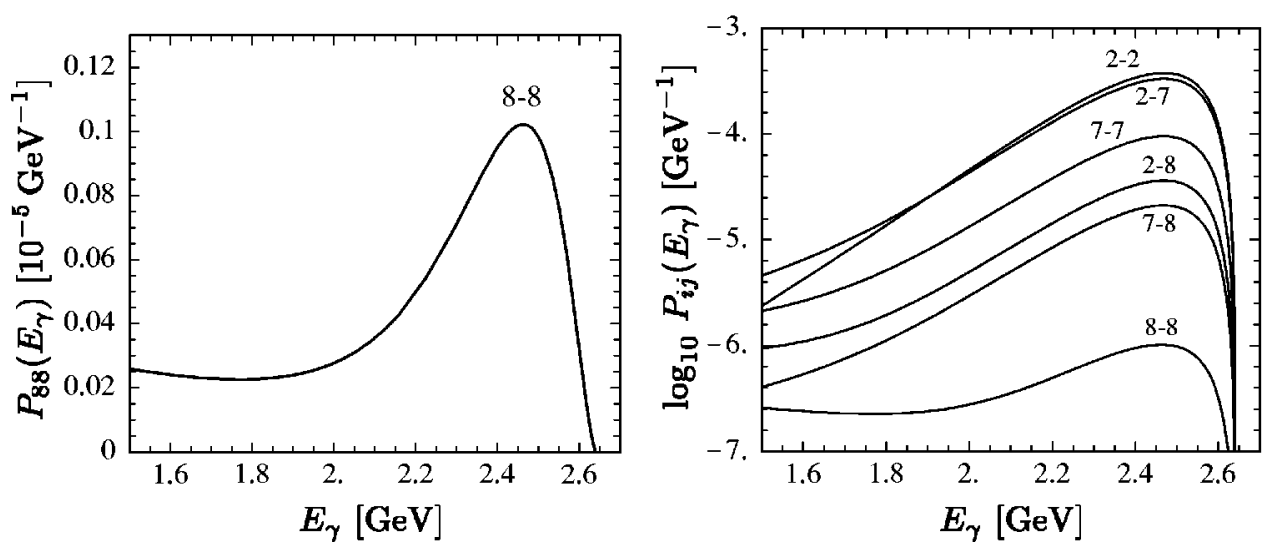

$B \rightarrow X_{s} \gamma$ decay. From Kagan and Neubert, 1999.
The impact of the higher-order corrections in $1 / m_{b}$ was quite recently investigated (Bauer et al., 2002; Leibovich et al., 2002).

The future aim should be to determine the shape function by using the high-precision measurements of the photon energy spectrum more precisely.

Moreover, the first and the second moments of the photon spectrum can be determined within the measurement of $B \rightarrow X_{s} \gamma$. These results can be used to extract values for the heavy-quark effective-theory parameters $\bar{\Lambda}$ and $\lambda_{1}$ (see Ligeti et al., 1999). CLEO has measured these moments and extracted, for example, $\bar{\Lambda}=0.35$ $\pm 0.08 \pm 0.10 \mathrm{GeV}$ from the first moment, where the first error is from experimental error and the second from the theoretical expression, in particular, from neglected higher-order terms (Chen et al., 2001).

A lower experimental cut in the photon energy spectrum within the measurement of $B \rightarrow X_{s} \gamma$ decreases the sensitivity to the parameters of the shape function and therefore the model dependence. With respect to this, the ideal energy cut would be $1.6 \mathrm{GeV}$ (see Fig. 10). But in this case a better understanding of the $\psi$ background would be mandatory. The intermediate $\psi$ background, namely, $B \rightarrow \psi X_{s}$ followed by $\psi \rightarrow X^{\prime} \gamma$, is more than 4 $\times 10^{-4}$ in the "total" branching ratio. With the present energy cut of $2.0 \mathrm{GeV}$, this contribution is suppressed and estimated to be less than $1.5 \%$; for $2.1 \mathrm{GeV}$ it is 0.6\% (Misiak, 2000b, 2000c).

\section{Experimental status of $B \rightarrow X_{s} \ell^{+} \ell^{-}$and $B \rightarrow X_{d} \gamma$}

The inclusive $B \rightarrow X_{s} \ell^{+} \ell^{-}$transition is also beginning to be accessible at the $B$ factories. BELLE and BABAR have already established measurements of the exclusive mode $B \rightarrow K \ell^{+} \ell^{-}$(Abe et al., 2002a, 2002e; Aubert et al., 2002e). The measurements are compatible with each other.

Quite recently, BELLE announced the first measurement of the inclusive $B \rightarrow X_{s} \ell^{+} \ell^{-}$mode based on a semi-inclusive analysis (Abe et al., 2001b; Kaneko et al., 2003). The hadronic system $X_{s}$ is reconstructed from a kaon with 0 to 4 pions (at most one $\pi^{0}$ ). The data sample used contains $65.4 \times 10^{6} B \bar{B}$ pairs.

The signal characteristics within this semi-inclusive analysis are determined by modeling the invariant-mass $M_{X_{s}}$ spectrum using the phenomenological model first

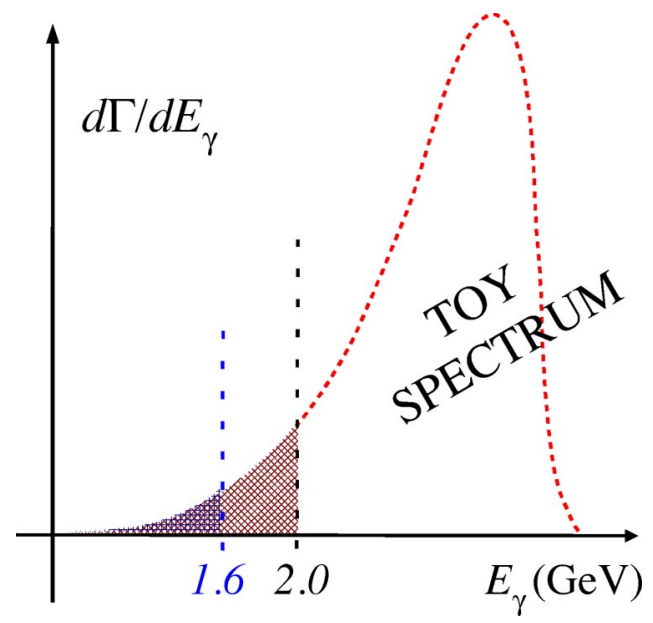

FIG. 10. (Color in online edition) Schematic photon spectrum of $B \rightarrow X_{s} \gamma$. 


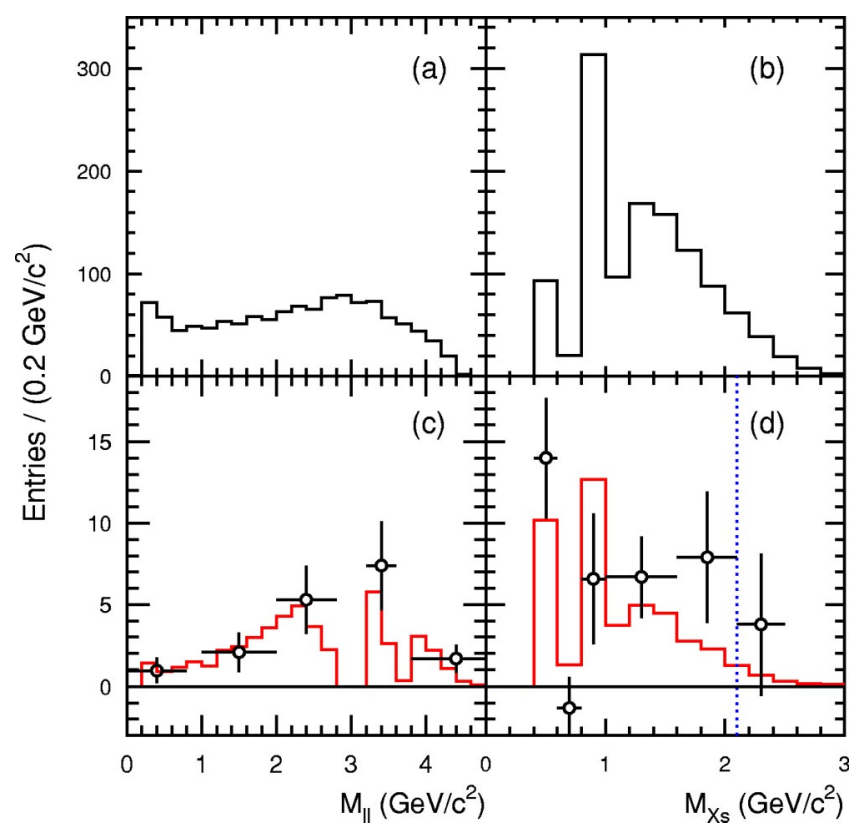

FIG. 11. (Color in online edition) Standard-model (SM) expectations and observed mass spectra: (a) expected dilepton spectrum; (b) expected recoil mass spectrum; (c) observed dilepton spectrum; (d) observed recoil mass spectrum (circles). The histograms in (c) and (d) show the standard-model expectations after all the selections are applied; histograms are normalized to the expected branching fractions. The dotted line in (d) indicates the $M_{X s}<2.1 \mathrm{GeV}$ requirement. From Kaneko et al., 2003.

proposed by Ali and Pietarinen (1979) and Altarelli et al. (1982). The reconstruction efficiencies of the signal are determined by the Monte Carlo samples based on this model, leading to a large part of the systematic uncertainty.

Most of the backgrounds do not produce the $B$ mass peak and can be estimated by a fit. But there are two peaking backgrounds. The first one is the process $B$ $\rightarrow X_{s} \pi^{+} \pi^{-}$, in which the two pions are misidentified as leptons. This background is estimated explicitly by reconstructing the $B \rightarrow X_{s} \pi^{+} \pi^{-}$and multiplying the yield by $\left(f_{\pi}^{\ell}\right)^{2}$, where $f_{\pi}^{\ell}$ is the probability of a pion faking a lepton measured in an independent data set. The charmonium decays $B \rightarrow \psi\left(\rightarrow \ell^{+} \ell^{-}\right) X_{s} \quad$ and $B$ $\rightarrow \psi^{\prime}\left(\rightarrow \ell^{+} \ell^{-}\right) X_{s}$ constitute the second source. They are vetoed by excluding lepton combinations whose invariant mass falls within a window around the nominal $\psi$ and $\psi^{\prime}$ mass.

The continuum background and the $B \bar{B}$ background, however, can be suppressed by kinematical constraints. Further suppression is achieved with methods similar to those used in analysis of $B \rightarrow X_{s} \gamma$.

Moreover, there is a cut used in the $X_{s}$ invariant-mass spectrum at $2.1 \mathrm{GeV}$. This removes a large part of the combinatorial background, while a model calculation determines that $(93 \pm 5) \%$ of the signal is within this experimental window (leading to an additional model dependence). Events with a dilepton mass $M_{\ell^{+} \ell^{-}}$less than $0.2 \mathrm{GeV}$ are also rejected in order to suppress elec-

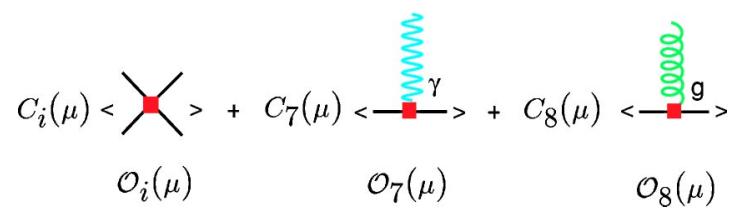

FIG. 12. (Color in online edition) Effective Hamiltonian in the case of $B \rightarrow X_{s, d} \gamma$.

tron pairs from $\pi^{0} \rightarrow e^{+} e^{-} \gamma$ and $\gamma \rightarrow e^{+} e^{-}$conversion.

A comparison of the histograms in Figs. 11(a) and 11(c) indicates that the efficiency within this measurement is much higher in the high-dilepton-mass region.

The uncertainty of this first measurement of the inclusive decay is still at the $30 \%$ level and in agreement with the standard-model expectations. One can expect much higher accuracy from the $B$ factories in the near future.

The inclusive decay $B \rightarrow X_{d} \gamma$ is also within reach of the high-luminosity $B$ factories. Such a measurement will rely on high statistics and on powerful methods for kaon-pion discrimination. At present only upper bounds on corresponding exclusive modes are available from CLEO (Coan et al., 2000), BELLE (Ushiroda et al., 2001; Abe et al., 2002d), and BABAR (Aubert et al., 2002c).

\section{PERTURBATIVE CALCULATIONS IN $B \rightarrow X_{s, d} \gamma$}

The inclusive decay $B \rightarrow X_{s} \gamma$ is a laboratory for perturbative QCD. Nonperturbative effects (see Sec. VI) play a subordinate role and are well under control thanks to the heavy-quark expansion. The dominant short-distance QCD corrections enhance the partonic decay rate $\Gamma\left(b \rightarrow X_{s}^{\text {parton }} \gamma\right)$ by a factor of more than 2 . The corresponding large logarithms of the form $\alpha_{s}^{n}\left(m_{b}\right) \ln ^{m}\left(m_{b} / M\right)$, where $M=m_{t}$ or $M=m_{W}$ and $m$ $\leqslant n$ (with $n=0,1,2, \ldots$ ), have to be summed with the help of the renormalization-group-improved perturbation theory, as presented in Sec. II.

The effective Hamiltonian relevant to $B \rightarrow X_{s} \gamma$ in the standard model reads

$$
H_{\mathrm{eff}}\left(B \rightarrow X_{s} \gamma\right)=-\frac{4 G_{F}}{\sqrt{2}} \lambda_{t} \sum_{i=1}^{8} C_{i}(\mu) \mathcal{O}_{i}(\mu),
$$

where $\mathcal{O}_{i}(\mu)$ are the relevant operators, $C_{i}(\mu)$ are the corresponding Wilson coefficients, which contain the complete top dependence and $W$-mass dependence (see Fig. 12), and $\lambda_{q}=V_{q b} V_{q s}^{*}$ with $V_{i j}$, the CKM matrix elements. The CKM dependence globally factorizes, ${ }^{1}$ if one works in the approximation $\lambda_{u}=0$. One neglects the operators with dimensions $>6$, which are suppressed by higher powers of $1 / m_{W}$.

Using the equations of motion for the operators, one arrives at the following basis of dimension- 6 operators

\footnotetext{
${ }^{1}$ This approximation is not used within the recent theoretical predictions.
} 
(Shifman et al., 1978; Grinstein et al., 1988, 1989; Chetyrkin et al., 1997):

$$
\begin{aligned}
& \mathcal{O}_{1}=\left(\bar{s} \gamma_{\mu} T^{a} P_{L} c\right)\left(\bar{c} \gamma^{\mu} T_{a} P_{L} b\right), \\
& \mathcal{O}_{2}=\left(\bar{s} \gamma_{\mu} P_{L} c\right)\left(\bar{c} \gamma^{\mu} P_{L} b\right), \\
& \mathcal{O}_{3}=\left(\bar{s} \gamma_{\mu} P_{L} b\right) \sum_{q}\left(\bar{q} \gamma^{\mu} q\right), \\
& \mathcal{O}_{4}=\left(\bar{s} \gamma_{\mu} T^{a} P_{L} b\right) \sum_{q}\left(\bar{q} \gamma^{\mu} T_{a} q\right), \\
& \mathcal{O}_{5}=\left(\bar{s} \gamma_{\mu} \gamma_{\nu} \gamma_{\rho} P_{L} b\right) \sum_{q}\left(\bar{q} \gamma^{\mu} \gamma^{\nu} \gamma^{\rho} q\right), \\
& \mathcal{O}_{6}=\left(\bar{s} \gamma_{\mu} \gamma_{\nu} \gamma_{\rho} T^{a} P_{L} b\right) \sum_{q}\left(\bar{q} \gamma^{\mu} \gamma^{\nu} \gamma^{\rho} T_{a} q\right), \\
& \mathcal{O}_{7}=\frac{e}{16 \pi^{2}} \bar{m}_{b}(\mu)\left(\bar{s} \sigma^{\mu \nu} P_{R} b\right) F_{\mu \nu}, \\
& \mathcal{O}_{8}=\frac{g_{s}}{16 \pi^{2}} \bar{m}_{b}(\mu)\left(\bar{s} \sigma^{\mu \nu} T^{a} P_{R} b\right) G_{\mu \nu}^{a} .
\end{aligned}
$$

In the dipole-type operators $\mathcal{O}_{7}$ and $\mathcal{O}_{8}, e$ and $F_{\mu \nu}\left(g_{s}\right.$ and $G_{\mu \nu}^{A}$ ) denote the electromagnetic (strong) coupling constant and field strength tensor, respectively. $T^{a}(a$ $=1,8)$ denote $\mathrm{SU}(3)$ color generators, and $P_{R, L}=(1$ $\left.\pm \gamma_{5}\right) / 2$.

The error of the leading-logarithmic (LL) result (Ciuchini et al., 1993, 1994; Misiak, 1993; Cella et al., 1994) was dominated by a large renormalization scale dependence at the $\pm 25 \%$ level, which already indicated the importance of the NLL series. By convention, the dependence on the renormalization scale $\mu_{b}$ is obtained by its variation between $m_{b} / 2$ and $2 m_{b}$. The former measurement of the CLEO Collaboration [see Eq. (3.1)] overlaps with the estimates based on LL calculations, and the experimental and theoretical errors are comparable. In view of the expected increase in the experimental precision, it became clear that a systematic inclusion of the NLL corrections was becoming necessary. Moreover, such a NLL program was also important in order to ensure the validity of renormalization-groupimproved perturbation theory in this specific phenomenological application.

This ambitious NLL enterprise was completed some years ago and was a joint effort of many different groups (Ali and Greub, 1991, 1995; Adel and Yao, 1994; Greub et al., 1996a; Chetyrkin et al., 1997). The theoretical error of the previous LL result was substantially reduced, to $\pm 10 \%$, and the central value of the partonic decay rate increased by about $20 \%$. All three steps to NLL precision listed below [Eq. (2.5)] involve rather difficult calculations (see Fig. 13).

- The most difficult part in Step 1 is the two-loop (or order- $\alpha_{s}$ ) matching of the dipole operators $\mathcal{O}_{7}$ and $\mathcal{O}_{8}$. It involves two-loop diagrams both in the full and in the effective-field theory. It was first worked out by Adel and Yao (1994). Since this is a crucial step in the

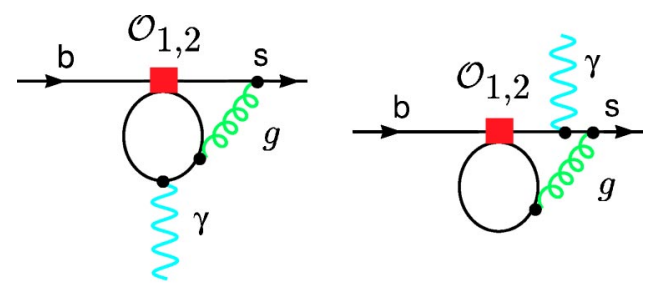

(a)

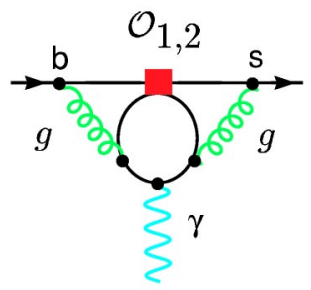

(b)

FIG. 13. (Color in online edition) Next-to-leading-logarithm contributions for Steps 2 and 3: (a) typical diagrams (finite parts) contributing to the matrix element of the operator $\mathcal{O}_{2}$ at the NLL level, Step 3; (b) typical diagram (infinite part) contributing to the NLL anomalous-dimension matrix, Step 2. See Fig. 2 for a typical diagram (finite part) contributing to the NLL matching calculation, Step 1.

NLL program, their findings were confirmed in a detailed recalculation using a different method (Greub and Hurth, 1997). Three further recalculations of this result, two complete (Ciuchini et al., 1998a; Bobeth et al., 2000a) and one partial (Buras et al., 1998a), were presented in the meanwhile, confirming the original results of Adel and Yao (1994). In order to match the dimension-6 operators $\mathcal{O}_{7}$ and $\mathcal{O}_{8}$, it is sufficient to extract the terms of order $m_{b}^{2} / M^{2}$ (M $\left.=m_{W}, m_{t}\right)$ from the standard-model matrix elements for $b \rightarrow s \gamma$ and $b \rightarrow s g$. Terms suppressed by additional powers of $m_{b} / M$ correspond to higher-dimensional operators in the effective theory. Greub and Hurth (1997) calculated the finite parts of the two-loop diagrams in the standard model by means of the wellknown method of asymptotic mass expansions, which naturally lead to a systematic expansion of Feynman diagrams in inverse powers of $M$.

- The order- $\alpha_{s}^{2}$ anomalous-dimension matrix (Step 2) has been worked out by Chetyrkin et al. (1997). In particular, the calculation of the elements $\gamma_{i 7}$ and $\gamma_{i 8}$ $(i=1, \ldots, 6)$ in the $O\left(\alpha_{s}^{2}\right)$ anomalous-dimension matrix involves a huge number of three-loop diagrams from which the pole parts (in the $d$-4 expansion) have to be extracted. This extraction was simplified by a clever decomposition of the scalar propagator (Chetyrkin et al., 1998a). Moreover, the number of necessary evanescent operators was reduced by a new choice of a basis of dimension-6 operators (Chetyrkin et al., 1998b). Using the matching result (Step 1), these authors obtained the NLL correction to the Wilson coefficient $C_{7}\left(\mu_{b}\right)$. Numerically, the LL and NLL 
values for $C_{7}\left(\mu_{b}\right)$ turn out to be rather similar; the NLL corrections to the Wilson coefficient $C_{7}\left(\mu_{b}\right)$ lead to a change of the $B \rightarrow X_{s} \gamma$ decay rate that does not exceed $6 \%$ in the $\overline{M S}$ scheme (Chetyrkin et al., 1997).

It should be stressed that the result of Step 2, in particular, the entries $\gamma_{i 7}$ and $\gamma_{i 8}(i=1, \ldots, 6)$ of the anomalous-dimension matrix to NLL precision, is the only part of the complete NLL enterprise that has not been confirmed by an independent group. An independent check of this important part of the NLL program is already on the way (Gambino et al., 2003).

- Step 3 basically consists of bremsstrahlung corrections and virtual corrections. While the bremsstrahlung corrections were worked out some time ago by Ali and Greub (1991, 1995) and were confirmed and extended by Pott (1996), a complete analysis of the virtual twoloop corrections (up to the contributions of the fourquark operators with very small coefficients) was presented by Greub et al. (1996b). This calculation involves two-loop diagrams, in which the full charmmass dependence has to be taken into account. By using Mellin-Barnes techniques in the Feynman parameter integrals, Greub, Hurth, and Wyler, (1996a, 1996b) obtained the result of these two-loop diagrams in the form

$c_{0}+\sum_{n=0,1,2, \ldots ; m=0,1,2,3} c_{n m}\left(\frac{m_{c}^{2}}{m_{b}^{2}}\right)^{n} \ln ^{m} \frac{m_{c}^{2}}{m_{b}^{2}}$,

where the quantities $c_{0}$ and $c_{n m}$ are independent of $m_{c}$. The convergence of the Mellin-Barnes series was proved; the practical convergence of the series [Eq. (4.3)] was also checked explicitly. Moreover, a finite result was obtained in the limit $m_{c} \rightarrow 0$, since there is no naked logarithm of $m_{c}^{2} / m_{b}^{2}$. This observation is of some importance in the $b \rightarrow d \gamma$ process, where the $u$-quark propagation in the loop is not CKM suppressed (see below). The main result of Step 3 consisted of a drastic reduction of the renormalization scale uncertainty in the decay rate from about $\pm 25 \%$ to about $\pm 6 \%$. The central value was shifted by about $20 \%$.

Greub et al. (1996a, 1996b) present these results also in the 't Hooft-Veltman scheme, which may be regarded as a first step towards a cross check of the complete NLL calculation prediction in a different renormalization scheme. Recently, the results of the $\mathcal{O}_{1,2}$ matrix elements in the $\overline{M S}$ scheme calculated by Greub, Hurth, and Wyler (1996a, 1996b) were confirmed by an independent group (Buras et al., 2001) with the help of the method of asymptotic expansions. Also, two further calculations confirmed these results with the help of a direct analytical method (Buras, Czarnecki, et al., 2002) and a numerical method (Ghinculov et al., 2003b). The direct analytical method also allowed control over the matrix elements of the penguin operators $\mathcal{O}_{3-6}$. As expected from the smallness of the corresponding Wilson coefficients, their effect on the branching ratio does not exceed $1 \%$.

Combining the NLL calculations of the three steps, the first practically complete theoretical prediction to NLL precision for the branching ratio of $B \rightarrow X_{s} \gamma$ was presented by Chetyrkin et al. (1997; see also Greub and Hurth, 1996):

$$
\mathcal{B}\left(B \rightarrow X_{s} \gamma\right)=(3.28 \pm 0.33) \times 10^{-4} .
$$

The theoretical error had two dominant sources, $\mu$ dependence, which was reduced to about $6 \%$, and $m_{c} / m_{b}$ dependence. This first theoretical NLL prediction already included the nonperturbative correction scaling with $1 / m_{b}^{2}$, which is rather small (at the $1 \%$ level; see Sec. VI). Surprisingly, the NLL predictions of Chetyrkin et al. (1997) and Greub and Hurth (1996) are almost identical to the present predictions [Eq. (4.3)] using the charm-pole mass, in spite of so many important additional refinements such as the electroweak two-loop corrections and the nonperturbative corrections, both of which will be discussed below.

Detailed studies of the two-loop electroweak corrections in the decay $B \rightarrow X_{s} \gamma$ were performed. Czarnecki and Marciano (1998) calculated some of the electroweak two-loop contributions, namely, contributions from fermion loops in gauge boson propagators $(\gamma$ and $W)$ and from short-distance photonic loop corrections. Moreover, it was observed that the on-shell value of the finestructure constant $1 / \alpha_{e m}=137$ is more appropriate for real photon emission than the value $1 / \alpha_{\text {em }}=(130.3$ \pm 2.3 ) used in previous analyses. The QED loop calculations of Czarnecki and Marciano (1998) confirmed this expectation. This change in $\alpha_{e m}$ leads to a reduction of $5 \%$ in the perturbative contribution. Strumia (1998) presented a calculation of the heavy top and the heavy Higgs corrections in the gaugeless limit $m_{W} \rightarrow 0$. Kagan and Neubert (1999) improved the QED analysis made by Czarnecki and Marciano (1998) by resumming the contributions of order $\alpha_{e m} \ln \left(\mu_{b} / M\right)\left[\alpha_{s} \ln \left(\mu_{b} / M\right)\right]^{n}$ to all orders, whereas Czarnecki and Marciano (1998) had included only the $n=0$ contribution. This resummation decreased the QED corrections. The same calculation was performed by Baranowski and Misiak (2000), taking into account the complete relevant set of operators. It was explicitly shown that the truncation of the operator basis in Kagan and Neubert (1999) turns out to be a correct approximation and that these corrections lead to only a $0.8 \%$ correction.

The most advanced analysis of the electroweak contributions to order $\alpha_{e m}\left[\alpha_{s} \ln (\mu / M)\right]^{n}$ was performed by Gambino and Haisch (2001). This included a two-loop matching to order $\alpha_{e m}$, the QED-QCD running of the Wilson coefficients down to the $b$ quark, and one- and two-loop QED matrix elements. While Gambino and Haisch (2000) had considered only the so-called purely electroweak contributions [terms proportional to $\alpha_{e m} / \sin ^{2}(\theta)$ or enhanced by powers of $\left.m_{t}\right]$, the same authors in 2001 presented the complete two-loop match- 

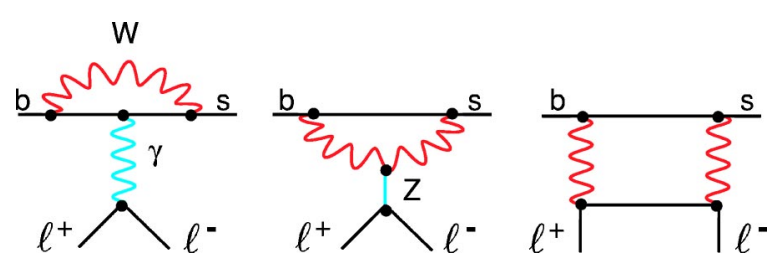

FIG. 14. (Color in online edition) One-loop contributions to the decay $B \rightarrow X_{s} \ell^{+} \ell^{-}$.

ing conditions to order $\alpha_{e m}$. It was shown that the electroweak two-loop corrections of order $\alpha_{e m}\left[\alpha_{s} \ln (\mu / M)\right]^{n}$ led, because of accidental cancellations, to a $1.6 \%$ reduction of the branching ratio of $B \rightarrow X_{s} \gamma$ only. Thus the electroweak corrections are well under control and can be seen to play a subordinate role.

It is clear that many parts of the perturbative calculations at the partonic level in the case of $B \rightarrow X_{s} \gamma$ can be applied to the cases $B \rightarrow X_{d} \gamma$ and $B \rightarrow X_{s} \ell^{+} \ell^{-}$. The latter case, however, needs some modifications-in particular, the operator basis gets enlarged, as will be discussed in the next subsection.

The perturbative QCD corrections in the decay $b$ $\rightarrow d \gamma$ can be treated in complete analogy to those in the decay $b \rightarrow s \gamma$ (Ali et al., 1998): the effective Hamiltonian is the same in the processes $b \rightarrow s \gamma$ and $b \rightarrow d \gamma$ up to the obvious replacement of the $s$-quark field by the $d$-quark field. But since $\xi_{u}=V_{u b} V_{u d}^{*}$ for $b \rightarrow d \gamma$ is not small with respect to $\xi_{t}=V_{t b} V_{t d}^{*}$ and $\xi_{c}=V_{c b} V_{c d}^{*}$, one also has to take into account the operators proportional to $\xi_{u}$. The matching conditions $C_{i}\left(m_{W}\right)$ and the solutions of the renormalization-group equations, yielding $C_{i}\left(\mu_{b}\right)$, coincide with those needed for the process $B \rightarrow X_{s} \gamma$.

The perturbative calculations at the partonic level of $B \rightarrow X_{s} \gamma$ can also be used for the partonic process $c$ $\rightarrow u \gamma$. As a FCNC process, it does not occur at the tree level in the standard model either. Moreover, GlashowIliopoulous-Maiani suppression is strong at one loop. The leading QCD logarithms are known to enhance the one-loop amplitude by more than one order of magnitude. It was shown by Greub, Hurth, Misiak, and Wyler (1996) that the amplitude increases by two further orders of magnitude after including the formally NLL QCD effects. So the $c \rightarrow u \gamma$ process is completely dominated by a two-loop term. However, this is only of theoretical interest, because the $\Delta S=0$ radiative decays of charmed hadrons remain dominated by the $c \rightarrow d \bar{d} u \gamma$ and $c \rightarrow s \bar{s} u \gamma$ subprocesses.

\section{PERTURBATIVE CALCULATIONS IN $B \rightarrow X_{s} \ell^{+} \ell^{-}$AND $B \rightarrow X_{s} \bar{\nu} \nu$}

In comparison with the $B \rightarrow X_{s} \gamma$ decay, the inclusive $B \rightarrow X_{s} \ell^{+} \ell^{-}$decay presents a complementary and also more complex test of the standard model, since different contributions add to the decay rate (Fig. 14).

It is particularly attractive because of kinematic observables such as the invariant dilepton-mass spectrum and forward-backward asymmetry. It is also dominated

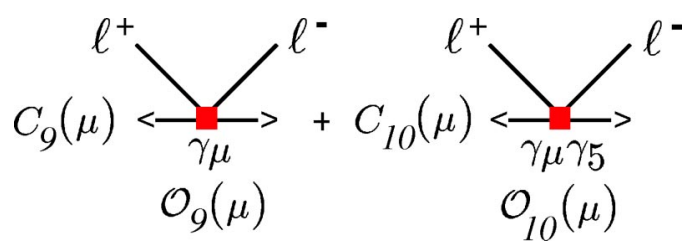

FIG. 15. (Color in online edition) Additional operators in the effective Hamiltonian in the case of $B \rightarrow X_{s, d} \ell^{+} \ell^{-}$.

by perturbative contributions, if one eliminates $c \bar{c}$ resonances with the help of kinematic cuts (see Sec. VII.D).

The effective Hamiltonian relevant to $B \rightarrow X_{s} \ell^{+} \ell^{-}$in the standard model reads

$$
H_{\mathrm{eff}}\left(B \rightarrow X_{s} \ell^{+} \ell^{-}\right)=-\frac{4 G_{F}}{\sqrt{2}} \lambda_{t} \sum_{i=1}^{10} C_{i}(\mu) \mathcal{O}_{i}(\mu) .
$$

Compared with the decay $B \rightarrow X_{s} \gamma$ [see Eq. (4.1)], the effective Hamiltonian (5.1) contains two additional operators $O\left(\alpha_{e m}\right)$ (see Fig. 15):

$$
\begin{aligned}
& \mathcal{O}_{9}=\frac{e^{2}}{16 \pi^{2}}\left(\bar{s} \gamma_{\mu} P_{L} b\right)\left(\bar{l} \gamma^{\mu} l\right), \\
& \mathcal{O}_{10}=\frac{e^{2}}{16 \pi^{2}}\left(\bar{s} \gamma_{\mu} P_{L} b\right)\left(\bar{l} \gamma^{\mu} \gamma_{5} l\right) .
\end{aligned}
$$

It turns out that the first large logarithm of the form $\ln \left(m_{b} / M\right)\left(M=m_{W}\right)$ already arises without gluons, because the operator $\mathcal{O}_{2}$ mixes into $\mathcal{O}_{9}$ at one loop via the diagram given in Fig. 16.

This possibility, which has no equivalent in the $b$ $\rightarrow s \gamma$ case, leads to the following ordering of contributions to the decay amplitude [which should be compared with Eqs. (2.2) and (2.3)]:

$$
\begin{aligned}
& {\left[\alpha_{e m} \ln \left(m_{b} / M\right)\right] \alpha_{s}^{n}\left(m_{b}\right) \ln ^{n}\left(m_{b} / M\right) \quad[\mathrm{LL}],} \\
& {\left[\alpha_{e m} \ln \left(m_{b} / M\right)\right] \alpha_{s}^{n+1}\left(m_{b}\right) \ln ^{n}\left(m_{b} / M\right) \quad[\mathrm{NLL}] .}
\end{aligned}
$$

Technically, to perform the resummation, it is convenient to transform these series into the standard form of Eq. (2.2). This can be achieved by redefining magnetic, chromomagnetic, and lepton-pair operators as follows (Misiak, 1993; Buras and Munz, 1995):

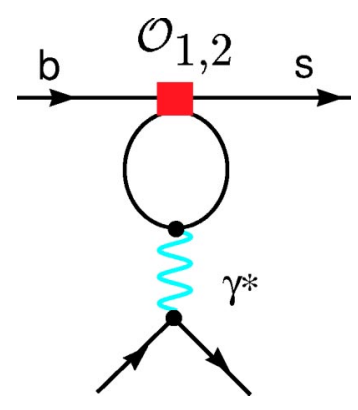

FIG. 16. (Color in online edition) Mixing of the operator $\mathcal{O}_{2}$ into $\mathcal{O}_{9}$ at one loop. 


$$
\mathcal{O}_{i}^{\text {new }}=\frac{16 \pi^{2}}{g_{s}^{2}} \mathcal{O}_{i}^{\text {old }}, \quad C_{i}^{\text {new }}=\frac{g_{s}^{2}}{(4 \pi)^{2}} C_{i}^{\text {old }} \quad(i=7, \ldots, 10)
$$

This redefinition enables one to proceed in the standard way, or as in $b \rightarrow s \gamma$, in the three calculational steps discussed at the end of Sec. II. At the high scale, the new Wilson coefficients can be computed at a given order in perturbation theory and expanded in powers of $\alpha_{s}$ :

$$
C_{i}^{\text {new }}=C_{i}^{(0)}+\frac{\alpha_{s}}{(4 \pi)} C_{i}^{(1)}+\frac{\alpha_{s}^{2}}{(4 \pi)^{2}} C_{i}^{(2)}+\cdots .
$$

Obviously, the Wilson coefficients of the new operators $\mathcal{O}_{7-10}$ at the high scale start only at order $\alpha_{s}$. Then the anomalous-dimension matrix has the canonical expansion in $\alpha_{s}$ and starts with a term proportional to $\alpha_{s}$ :

$$
\gamma^{n e w}=\frac{\alpha_{s}}{4 \pi} \gamma^{(0)}+\frac{\alpha_{s}^{2}}{(4 \pi)^{2}} \gamma^{(1)}+\frac{\alpha_{s}^{3}}{(4 \pi)^{3}} \gamma^{(2)}+\cdots .
$$

In particular, after the reshufflings in Eq. (5.4), the oneloop mixing of the operator $\mathcal{O}_{2}$ with $\mathcal{O}_{9}$ formally appears at order $\alpha_{s}$.

The last of the three steps, however, requires some care: among the new operators with a nonvanishing treelevel matrix element, only $\mathcal{O}_{9}$ has a nonvanishing coefficient at the LL level. Therefore, at this level, only the tree-level matrix element of this operator $\left(\left\langle\mathcal{O}_{9}\right\rangle\right)$ has to be included. At NLL accuracy, the QCD one-loop contributions to $\left\langle\mathcal{O}_{9}\right\rangle$, the tree-level contributions to $\left\langle\mathcal{O}_{7}\right\rangle$ and $\left\langle\mathcal{O}_{10}\right\rangle$, and the electroweak one-loop matrix elements of the four-quark operators all have to be calculated. Finally, at next-to-next-to-leading-log (NNLL) precision, one should, in principle, take into account the QCD two-loop corrections to $\left\langle\mathcal{O}_{9}\right\rangle$, the QCD one-loop corrections to $\left\langle\mathcal{O}_{7}\right\rangle$ and $\left\langle\mathcal{O}_{10}\right\rangle$, and the QCD corrections to the electroweak one-loop matrix elements of the fourquark operators.

The present status of these perturbative contributions to decay rate and forward-backward asymmetry of $B$ $\rightarrow X_{s} \ell^{+} \ell^{-}$is that the complete NLL contributions to the decay amplitude have been found by Misiak (1993) and Buras and Munz (1995). Since the LL contribution to the rate turns out to be numerically rather small, NLL terms represent an $O(1)$ correction to this observable. On the other hand, since a nonvanishing forwardbackward asymmetry is generated by the interference of vector $\left(\sim \mathcal{O}_{7,9}\right)$ and axial-vector $\left(\sim \mathcal{O}_{10}\right)$ leptonic currents, the LL amplitude leads to a vanishing result and NLL terms represent the lowest nontrivial contribution to this observable.

In view of the forthcoming precise measurements at the $B$ factories, a computation of NNLL terms in $B$ $\rightarrow X_{s} \ell^{+} \ell^{-}$is needed if one aims at the same numerical accuracy as achieved by the NLL analysis of $B \rightarrow X_{s} \gamma$. Large parts of the latter can be applied in the NNLL calculation of $B \rightarrow X_{s} \ell^{+} \ell^{-}$. But this is not the full story.

- (Step 1) The full computation of initial conditions to NNLL precision is presented by Bobeth et al. (2000b). The authors performed the two-loop matching for all
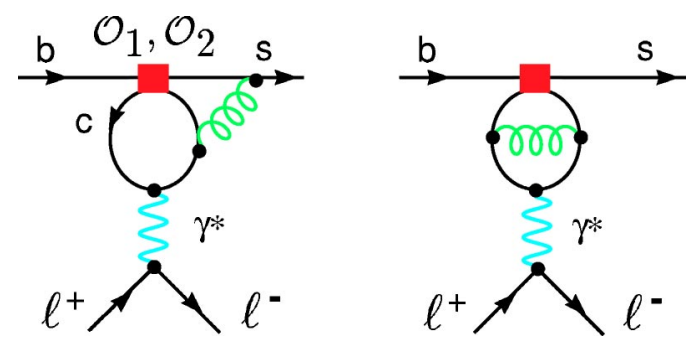

FIG. 17. (Color in online edition) Typical diagrams (finite parts) contributing to the matrix element of the operator $\mathrm{O}_{2}$ at the NNLL level (Step 3).

the operators relevant to $b \rightarrow s \ell^{+} \ell^{-}$[including a confirmation of the $b \rightarrow s \gamma$ NLL matching results of Adel and Yao (1994) and Greub and Hurth (1997)]. The inclusion of this NNLL contribution removes the large matching scale uncertainty (around 16\%) of the NLL calculation of the $b \rightarrow s \ell^{+} \ell^{-}$decay rate.

- (Step 2) Thanks to reshuffling of the LL series, most of the NNLL contributions to the anomalousdimension matrix can be derived from the NLL analysis of $b \rightarrow s \gamma$. In particular, the three-loop mixing of the four-quark operators $\mathcal{O}_{1-6}$ into $\mathcal{O}_{7}$ and $\mathcal{O}_{8}$ can be taken from Chetyrkin et al. (1997), which allows an evaluation of the matrix element $U_{72}^{(2)}$ [using the usual convention $\left.C_{i}\left(\mu_{b}\right)=U_{i j} C_{j}\left(\mu_{W}\right)\right]$. The only missing piece for a full NNLL analysis of the $b \rightarrow s \ell^{+} \ell^{-}$decay rate is the matrix element $U_{92}^{(2)}$ (see Fig. 18 below).

Bobeth et al. (2000b) estimated that the numerical influence of $U_{92}^{(2)}$ on the branching ratio of $b \rightarrow s \ell^{+} \ell^{-}$is small, which was confirmed by an explicit calculation in the meanwhile by Gambino et al. (2003). Interestingly, since the forward-backward asymmetry has no contributions proportional to $\left|\left\langle\mathcal{O}_{9}\right\rangle\right|^{2}$, this missing term is not needed for a NNLL analysis of this observable.

- (Step 3) Within the $B \rightarrow X_{s} \gamma$ calculation at NLL, the two-loop matrix elements of the four-quark operator $\mathcal{O}_{2}$ for an on-shell photon were calculated by Greub, Hurth, and Wyler (1996a, 1996b) using Mellin-Barnes techniques. This calculation was extended by Asatryan et al. (2001) to the case of an off-shell photon (see Fig. 17) with the help of a double Mellin-Barnes representation, which corresponds to a NNLL contribution relevant to the decay $B \rightarrow X_{s} \ell^{+} \ell^{-}$. The validity of these analytical results given by Asatryan et al. (2001) is restricted to small dilepton masses $q_{\ell^{+} \ell^{-}}^{2} / m_{b}^{2}<0.25$. An independent numerical check of these results has been performed by Ghinculov et al. (2003b). Moreover, the NNLL calculation of Ghinculov et al. (2003b) is also valid for high-dilepton masses, for which the experimental methods have an efficiency much higher than that at low-dilepton masses. Step 3 also includes the bremsstrahlung contributions which were calculated for the dilepton-mass spectrum (symmetric part) and for forward-backward asymmetry by Asatryan et al. (2002) and Ghinculov et al. 
(a)

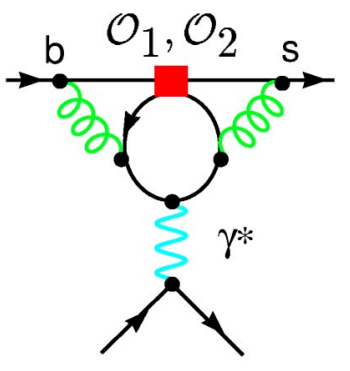

(b)

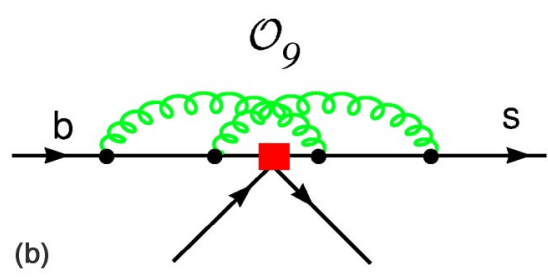

FIG. 18. (Color in online edition) Parts still missing in a complete canonical NNLL analysis of the dilepton mass spectrum. (a) Typical diagram (infinite part) contributing to the NNLL mixing $U_{92}^{(2)}$, Step 2. (b) Typical diagram (finite piece) contributing to the NNLL matrix element of the operator $\mathcal{O}_{9}$, Step 3.

(2003a). In the low-dilepton spectrum, these matrixelement calculations reduce the error corresponding to the uncertainty of the low-scale dependence from $\pm 13 \%$ down to $\pm 6.5 \%$.

In principle, a complete NNLL calculation of the $B$ $\rightarrow X_{s} \ell^{+} \ell^{-}$rate would also require the calculation of the renormalization-group-invariant two-loop matrix element of the operator $\mathcal{O}_{9}$ (see Fig. 18). But its impact on the dilepton-mass spectrum is expected to be small. Similar to the missing piece of the anomalousdimension matrix, this (scale-independent) contribution does not enter the forward-backward asymmetry at NNLL accuracy.

As anticipated, the canonical LL expansion is numerically not well justified, since the formally leading $O\left(1 / \alpha_{s}\right)$ term in $C_{9}$ is numerically close to the $O(1)$ term. For this reason, it has been proposed (Asatryan et al., 2001) that we use a different counting rule, in which the $O\left(1 / \alpha_{s}\right)$ term of $C_{9}$ is treated as $O(1)$. This approach, although it cannot be consistently extended at higher orders, seems to be well justified at the present status of the calculation. Within this approach, the two missing ingredients for a NNLL calculation of the dilepton-mass spectrum (see Fig. 18) would be of higher order.

The decay $B \rightarrow X_{s, d} \nu \bar{\nu}$ is induced by $Z^{0}$ penguin and box diagrams (see Fig. 19). The main difference to the semileptonic decay $B \rightarrow X_{s, d} \ell^{+} \bar{\ell}^{-}$is the absence of a photon penguin contribution. The latter implies only a logarithmic Glashow-Iliopoulous-Maiani (GIM) suppression, while the former contributions have a quadratic GIM suppression. As a consequence, the depen-

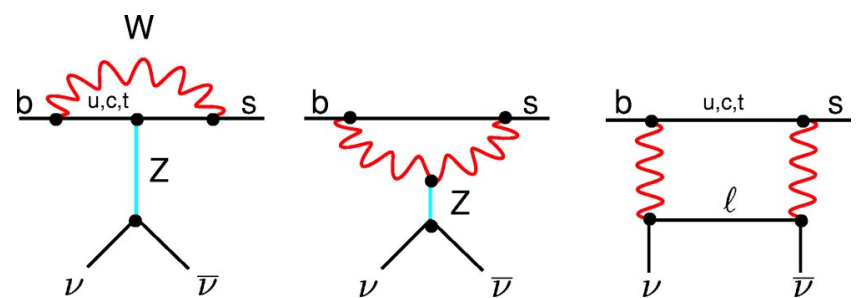

FIG. 19. (Color in online edition) One-loop contributions to the decay $B \rightarrow X_{s} \nu \bar{\nu}$.

dence of the amplitude of the decay $B \rightarrow X_{s, d} \nu \bar{\nu}$ on the up- and charm-quark masses is negligible. ${ }^{2}$

The effective Hamiltonian reads

$$
\begin{aligned}
H_{\text {eff }}(B & \left.\rightarrow X_{s} \nu \bar{\nu}\right) \\
= & -\frac{4 G_{F}}{\sqrt{2}} \frac{\alpha}{2 \pi \sin ^{2} \Theta_{W}} V_{t b} V_{t s}^{*} C\left(m_{t}^{2} / m_{W}^{2}\right) \\
& \times\left(\bar{s} \gamma_{\mu} P_{L} b\right)\left(\bar{\nu} \gamma^{\mu} P_{L} \nu\right)+\text { H.c. }
\end{aligned}
$$

For the decay $B \rightarrow X_{d} \nu \bar{\nu}$ the obvious changes have to be made.

The hard (quadratic) GIM mechanism leads to $C\left(m_{c}^{2} / m_{W}^{2}\right) / C\left(m_{t}^{2} / m_{W}^{2}\right) \approx O\left(10^{-3}\right)$. Moreover, the corresponding CKM factors in the top and the charm contributions are both of order $\lambda^{2}$. Therefore the charm contribution (and also the up-quark contribution) can safely be neglected.

The NLL QCD contributions to the partonic decay rate are presented by Buchalla and Buras (1993) and Misiak and Urban (1999). The perturbative error, that is the one due to the renormalization scale, was reduced from $O(10 \%)$ at the LL level to $O(1 \%)$ at the NLL level.

\section{NONPERTURBATIVE CONTRIBUTIONS}

\section{A. Inclusive decay rates of $B$ mesons}

In contrast to the exclusive rare $B$ decays, the inclusive ones are theoretically clean observables and dominated by the partonic contributions. Bound-state effects of the final states are eliminated by averaging over a specific sum of hadronic states. Moreover, long-distance effects of the inital state are accounted for through the heavy-mass expansion, in which the inclusive decay rate of a heavy $B$ meson is calculated using an expansion in inverse powers of the $b$-quark mass (Chay et al., 1990; Bigi et al., 1992, 1997; Manohar and Wise, 2000).

This heavy-mass expansion is now a well-known method for calculating the inclusive decay rates of a hadron containing a heavy quark, especially a $b$ quark.

\footnotetext{
${ }^{2}$ The notion that "the amplitude is dominated by the topquark contribution" is slightly imprecise because of the presence of GIM-canceling mass-independent contributions.
} 
The optical theorem relates the inclusive decay rate of a hadron $H_{b}$ to the imaginary part of certain forwardscattering amplitudes,

$$
\Gamma\left(H_{b} \rightarrow X\right)=\frac{1}{2 m_{H_{b}}} \operatorname{Im}\left\langle H_{b}|\mathbf{T}| H_{b}\right\rangle,
$$

where the transition operator $\mathbf{T}$ is given by $\mathbf{T}$ $=i \int d^{4} x T\left[H_{\mathrm{eff}}(x) H_{\mathrm{eff}}(0)\right]$. The insertion of a complete set of states, $X\rangle\langle X$, leads to the standard formula for the decay rate:

$$
\begin{aligned}
\Gamma\left(H_{b} \rightarrow X\right)= & \frac{1}{2 m_{H_{b}}} \sum_{X}(2 \pi)^{4} \delta^{4}\left(p_{i}-p_{f}\right) \\
& \times\left|\left\langle X\left|H_{\mathrm{eff}}\right| H_{b}\right\rangle\right|^{2} .
\end{aligned}
$$

It is then possible to construct an operator-product expansion of the operator $\mathbf{T}$, which gets expressed as a series of local operators-suppressed by powers of the $b$-quark mass and written in terms of the $b$-quark field:

$$
\stackrel{O P E}{\mathbf{T}}=\frac{1}{m_{b}}\left(\mathcal{O}_{0}+\frac{1}{m_{b}} \mathcal{O}_{1}+\frac{1}{m_{b}^{2}} \mathcal{O}_{2}+\cdots\right) .
$$

This construction is based on the parton-hadron duality (Shifman, 2000; Bigi and Uraltsev, 2001), using the facts that the sum is done over all exclusive final states and that the energy release in the decay is large with respect to the QCD scale, $\Lambda_{Q C D} \ll m_{b}$.

With the help of the heavy-quark effective theory, that is, the new heavy-quark spin-flavor symmetries arising in the heavy-quark limit $m_{b} \rightarrow \infty$ (Isgur and Wise, 1989, 1990; Neubert, 1994b), the hadronic matrix elements within the operator-product expansion, $\left\langle H_{b}\left|\mathcal{O}_{i}\right| H_{b}\right\rangle$, can be further simplified.

Two crucial observations within this well-defined procedure are important for the application to the inclusive rare $B$ decays. First, the free-quark model turns out to be the first term in the constructed expansion in powers of $1 / m_{b}$ and therefore the dominant contribution. This contribution can be calculated in perturbative QCD. Second, in the applications to inclusive rare $B$ decays one finds no correction of order $1 / m_{b}$ to the free-quark model approximation, and the corrections to the partonic decay rate start with $1 / m_{b}^{2}$ only. The latter fact implies a rather small numerical impact of the nonperturbative corrections on the decay rate of inclusive modes.

\section{B. Nonperturbative corrections to $B \rightarrow X_{s, d} \gamma$ and $B \rightarrow X_{s} \ell^{+} \ell^{-}$}

These techniques can be used directly in the decay $B \rightarrow X_{s} \gamma$, in order to single out nonperturbative corrections to the branching ratio. They are also applicable to the case of $B \rightarrow X_{d} \gamma$ and, with some modifications, to the case of $B \rightarrow X_{s} \ell^{+} \ell^{-}$.

If one neglects perturbative QCD corrections and assumes that the decay $B \rightarrow X_{s} \gamma$ is due solely to the operator $\mathcal{O}_{7}$, then one has to consider the time-ordered product $\mathrm{TO}_{7}^{+}(x) \mathcal{O}_{7}(0)$ (see Fig. 20). Using the operatorproduct expansion for $T \mathcal{O}_{7}^{+}(x) \mathcal{O}_{7}(0)$ and heavy-quark

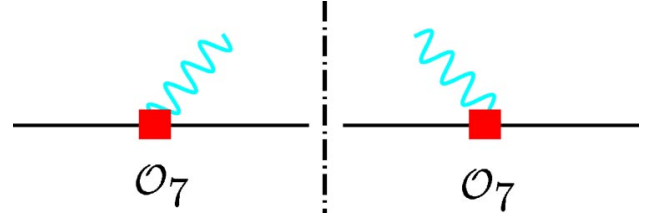

FIG. 20. (Color in online edition) Relevant cut diagram for the $\left(\mathcal{O}_{7}, \mathcal{O}_{7}\right)$ contribution.

effective theory methods as discussed above, the decay width $\Gamma\left(B \rightarrow X_{s} \gamma\right)$ reads (Falk et al., 1994; Ali et al., 1997) (modulo higher terms in the $1 / m_{b}$ expansion)

$$
\begin{aligned}
& \Gamma_{B \rightarrow X_{s} \gamma}^{\left(\mathcal{O}_{7}, \mathcal{O}_{7}\right)}=\frac{\alpha G_{F}^{2} m_{b}^{5}}{32 \pi^{4}}\left|V_{t b} V_{t s}\right|^{2} C_{7}^{2}\left(m_{b}\right)\left(1+\frac{\delta_{r a d}^{N P}}{m_{b}^{2}}\right), \\
& \delta_{r a d}^{N P}=\frac{1}{2} \lambda_{1}-\frac{9}{2} \lambda_{2},
\end{aligned}
$$

where $\lambda_{1}$ and $\lambda_{2}$ are the heavy-quark effective theory parameters for the kinetic energy and the chromomagnetic moment operators. Using $\lambda_{1}=-0.5 \mathrm{GeV}^{2}$ and $\lambda_{2}$ $=0.12 \mathrm{GeV}^{2}$, one gets $\delta_{\text {rad }}^{N P} \simeq-3 \%$.

The $B \rightarrow X_{s} \gamma$ decay width is usually normalized by the semileptonic one. The semileptonic decay width gets $1 / m_{b}^{2}$ corrections, which are also negative:

$$
\delta_{\text {semilept }}^{N P}=\frac{1}{2} \lambda_{1}-\left[\frac{3}{2}-\frac{6(1-z)^{4}}{g(z)}\right] \lambda_{2},
$$

where $g(z)$ is a phase-space factor and $z$ the ratio $m_{c, p o l e}^{2} / m_{b, p o l e}^{2}$.

The nonperturbative corrections scaling with $1 / m_{b}^{2}$ tend to cancel in the branching ratio $\mathcal{B}\left(B \rightarrow X_{s} \gamma\right) / \mathcal{B}(B$ $\left.\rightarrow X_{c} \ell \nu\right)$, and only about $1 \%$ remain: the heavy-quark effective theory parameter $\lambda_{1}$ cancels out in the ratio and the heavy-quark effective theory parameter $\lambda_{2}$ is known from $B^{*}-B$ mass splitting,

$$
\lambda_{2}=\frac{1}{4}\left(m_{B^{*}}^{2}-m_{B}^{2}\right) .
$$

Voloshin (1997) considers the nonperturbative effects that arise when including the operator $\mathcal{O}_{2}$. This effect is generated by the diagram in Fig. 21(a) (and by one, not shown, in which the gluon and the photon are interchanged); $g$ is a soft gluon interacting with the charm quarks in the loop. Up to a characteristic Lorentz structure, this loop is given by the integral

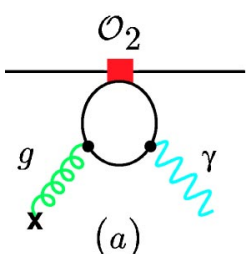

(a)

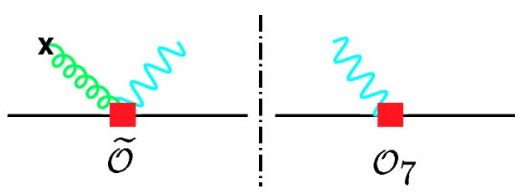

(b)
FIG. 21. (Color in online edition) The operator $\widetilde{\mathcal{O}}$. (a) Feynman diagram from which the operator $\widetilde{\mathcal{O}}$ arises. (b) Relevant cut diagram for the $\left(\widetilde{\mathcal{O}}, \mathcal{O}_{7}\right)$ interference. 


$$
\int_{0}^{1} d x \int_{0}^{1-x} d y \frac{x y}{m_{c}^{2}-k_{g}^{2} x(1-x)-2 x y k_{g} k_{\gamma}} .
$$

Since the gluon is soft, i.e., $k_{g}^{2}, k_{g} k_{\gamma} \approx \Lambda^{Q C D} m_{b} / 2 \ll m_{c}^{2}$, the integral can be expanded in $k_{g}$. The (formally) leading operator, denoted by $\widetilde{\mathcal{O}}$, is

$$
\begin{aligned}
\widetilde{\mathcal{O}}= & \frac{G_{F}}{\sqrt{2}} V_{c b} V_{c s}^{*} C_{2} \frac{e Q_{c}}{48 \pi^{2} m_{c}^{2}} \\
& \times \bar{s} \gamma_{\mu}\left(1-\gamma_{5}\right) g_{s} G_{\nu \lambda} b \epsilon^{\mu \nu \rho \sigma} \partial^{\lambda} F_{\rho \sigma} .
\end{aligned}
$$

Working out the cut diagram shown in Fig. 21(b), one then obtains the nonperturbative contribution $\Gamma_{B \rightarrow X_{s} \gamma}^{\left(\tilde{\mathcal{O}}, \mathcal{O}_{7}\right)}$ to the decay width, which is due to $\left(\mathcal{O}_{2}, \mathcal{O}_{7}\right)$ interference. Normalizing this contribution by the LL partonic width, one obtains (Buchalla et al., 1998)

$$
\frac{\Gamma_{B \rightarrow X_{s} \gamma}^{\left(\tilde{\mathcal{O}}, \mathcal{O}_{7}\right)}}{\Gamma_{b \rightarrow s \gamma}^{L L}}=-\frac{1}{9} \frac{C_{2}}{C_{7}} \frac{\lambda_{2}}{m_{c}^{2}} \simeq+0.03 .
$$

Since the expansion parameter is $m_{b} \Lambda_{Q C D} / m_{c}^{2} \approx 0.6$ (rather than $\Lambda_{Q C D}^{2} / m_{c}^{2}$ ), it is not a priori clear whether, formally, higher-order terms in the $1 / m_{c}$ expansion are numerically suppressed. More detailed investigations (Grant et al., 1997; Ligeti et al., 1997; Buchalla et al., 1998) have shown that higher-order terms are indeed suppressed because the corresponding expansion coefficients are small.

The analogous $1 / m_{c}^{2}$ effect has been found independently in the exclusive mode $B \rightarrow K^{*} \gamma$ by Khodjamirian et al. (1997). Numerically, the effect there is also at the few percent level.

As was emphasized by Misiak (2000a), an analogous systematic analysis of terms such as $\Gamma_{B \rightarrow X_{s} \gamma}^{\left(\mathrm{O}_{2}, \mathcal{O}_{2}\right)}$ at first order in $\alpha_{s}\left(m_{b}\right)$ is still missing. Rigorous techniques such as the operator-product expansion do not seem to be applicable in this case.

The analysis of the $1 / m_{b}^{2}$ and $1 / m_{c}^{2}$ effects has been extended to the decay $B \rightarrow X_{s} \ell^{+} \ell^{-}$by Falk $e$ al. (1994), Ali et al. (1997), Chen et al. (1997), Buchalla and Isidori (1998), Buchalla et al. (1998), and Bauer and Burrell (2000). These effects can be calculated quite analogously to those in the decay $B \rightarrow X_{s} \gamma$. As was first noticed by Ali et al. (1997), the relative $\Lambda_{Q C D}^{2} / m_{b}^{2}$ correction diverges in the high- $q_{\ell^{+} \ell^{-}}$end point, which indicates the breakdown of the heavy-mass expansion. Nevertheless, integrated quantities are still well defined.

There are also on-shell $c \bar{c}$ resonances, which have to be taken into account. For example, while in the decay $B \rightarrow X_{s} \gamma$ (on-shell photon) the intermediate $\psi$ background, namely, $B \rightarrow \psi X_{s}$ followed by $\psi \rightarrow X^{\prime} \gamma$, is suppressed for a high-energy cut $E_{\gamma}$ and can be subtracted from the $B \rightarrow X_{s} \gamma$ decay rate (see Sec. III.A), the $c \bar{c}$ resonances show up as large peaks in the dilepton invariant-mass spectrum in the decay $B \rightarrow X_{s} \ell^{+} \ell^{-}$(offshell photon; Deshpande et al., 1989; Lim et al., 1989). These resonances can be removed by appropriate kinematic cuts in the invariant-mass spectrum.
The nonperturbative contributions in the decay $B$ $\rightarrow X_{d} \gamma$ can also be treated analogously to the ones in the decay $B \rightarrow X_{s} \gamma$. The power corrections in $1 / m_{b}^{2}$ and $1 / m_{c}^{2}$ (besides the CKM factors) are the same for the two modes. But the long-distance contributions from the intermediate $u$ quark in the penguin loops are different. These are suppressed in the $B \rightarrow X_{s} \gamma$ mode by the unfavorable CKM matrix elements. In $B \rightarrow X_{d} \gamma$, there is no CKM suppression and one has to include the longdistance intermediate $u$-quark contributions.

Naively, one could expect that these contributions from up-quark loops scale with $1 / m_{u}^{2}$. However, following the approach of Buchalla et al. (1998), one can easily show that the general vertex function cannot in that case be expanded in the parameter $t=k \cdot q / m_{u}^{2}$ (where $k$ and $q$ are the gluon and photon momenta, respectively). But the expansion in inverse powers of $t$ is reasonable. The leading term in this expansion scales like $t^{-1} \sim m_{u}^{2} / k_{g} k_{\gamma}$ and therefore cancels the factor $1 / m_{u}^{2}$ in the prefactor [see the analogous $1 / m_{c}^{2}$ factor in Eq. (6.8)] and one can get a suppression factor $\left(\Lambda_{Q C D}^{2} / m_{u}^{2}\right) \cdot\left(m_{u}^{2} / k_{g} k_{\gamma}\right)$. Thus although the expansion in inverse powers in $t$ induces nonlocal operators, one explicitly finds that the leading term scales with $\Lambda_{Q C D} / m_{b}$. This indicates no large long-distance intermediate $u$-quark contributions.

Model calculations, based on vector-meson dominance, also suggest this conclusion (Ricciardi, 1995; Deshpande et al., 1996). Furthermore, estimates of the long-distance contributions in exclusive decays $B \rightarrow \rho \gamma$ and $B \rightarrow \omega \gamma$ in the light-cone sum-rule approach do not exceed 15\% (Ali and Braun, 1995; Khodjamirian et al., 1995).

Finally, it must be stressed that there is no spurious enhancement of the form $\left(m_{u} / \mu_{b}\right)$ in the perturbative contribution to the matrix elements of the four-quark operators, as shown by the explicit calculation of Greub, Hurth, and Wyler (1996a, 1996b; see also Abud et al., 1998). In other words, the limit $m_{u} \rightarrow 0$ can be taken.

All these observations exclude very large longdistance intermediate $u$-quark contributions in the decay $B \rightarrow X_{d} \gamma$.

\section{PHENOMENOLOGY}

\section{A. Standard-model prediction of $B \rightarrow X_{s} \gamma$}

The theoretical prediction for the partonic $b$ $\rightarrow X_{s}^{\text {parton }} \gamma$ decay rate is usually normalized by the semileptonic decay rate in order to get rid of uncertainties related to the CKM matrix elements and the fifth power of the $b$-quark mass. Moreover, an explicit lower cut on the photon energy in the bremsstrahlung correction has to be made:

$$
R_{\text {quark }}(\delta)=\frac{\Gamma[b \rightarrow s \gamma]+\Gamma[b \rightarrow s \gamma \text { gluon }]_{\delta}}{\Gamma\left[b \rightarrow X_{c} e \bar{\nu}_{e}\right]},
$$

where the subscript $\delta$ means that only photons with energy $E_{\gamma}>(1-\delta) E_{\gamma}^{\max }=(1-\delta) m_{b} / 2$ are counted. The ratio $R_{\text {quark }}$ is divergent in the limit $\delta \rightarrow 1$, owing to 
the soft-photon divergence in the subprocess $b$ $\rightarrow s \gamma$ gluon. In this limit only the sum of $\Gamma[b \rightarrow s \gamma]$, $\Gamma[b \rightarrow$ sluon $]$, and $\Gamma[b \rightarrow$ s $\gamma$ gluon $]$ is a reasonable physical quantity, in which all divergences cancel out. Kagan and Neubert (1999) showed that the theoretical result is rather sensitive to the unphysical soft-photon divergence; the choice $\delta=0.90$ was suggested as the definition of the "total" decay rate.

It is suggestive to give up the concept of a "total" decay rate of $b \rightarrow s \gamma$ and to compare theory and experiment using the same energy cut. Then again the theoretical uncertainty regarding the photon energy spectrum mentioned in Sec. III.B would occur naturally in the theoretical prediction.

Using the measured semileptonic branching ratio $\mathcal{B}_{\text {exp }}^{\text {sl }}$, one finds the branching ratio $\mathcal{B}\left(B \rightarrow X_{s} \gamma\right)$ given by

$$
\mathcal{B}\left(B \rightarrow X_{s} \gamma\right)=R_{\text {quark }} \times \mathcal{B}_{\text {exp }}^{\text {sl }}\left(1+\Delta_{\text {nonpert }}\right),
$$

where the nonperturbative corrections scaling with $1 / m_{b}^{2}$ and $1 / m_{c}^{2}$, summed in $\Delta_{\text {nonpert }}$ (see Sec. VI), have numerical effects of $+1 \%$ and $+3 \%$, respectively, on the branching ratio only. For a comparison with the ALEPH measurement [Eq. (3.3)], the measured semileptonic branching ratio $\mathcal{B}\left(H_{b} \rightarrow X_{c, u} \ell \nu\right)$ should be used consistently. This leads to a slightly larger theoretical prediction for the LEP experiments.

Including only the resummed QED corrections and the nonperturbative corrections discussed in Sec. VI, and using the on-shell value of $\alpha_{e m}$ and the charm-pole mass, one ends up with the following theoretical prediction for the $B \rightarrow X_{s} \gamma$ branching ratio (Greub and Hurth, 1999):

$$
\mathcal{B}\left(B \rightarrow X_{s} \gamma\right)=(3.32 \pm 0.30) \times 10^{-4},
$$

where the error has two sources, namely, the uncertainty regarding the scale dependencies and the uncertainty due to the input parameters. In the latter the uncertainty due to the parameter $m_{c} / m_{b}$ is dominant. This prediction almost coincides with the prediction of Kagan and Neubert (1999).

Gambino and Misiak (2001) made two important observations. First it was shown that the charm-loop contribution to the decay $B \rightarrow X_{s} \gamma$ is numerically dominant and very stable under logarithmic QCD corrections, and that the strong enhancement of the branching ratio by QCD logarithms is mainly due to the $b$-quark mass evolution in the top-quark sector. This leads to better control over the residual scale dependence at NLL.

Second, quark mass effects within the decay $B \rightarrow X_{s} \gamma$ were further analyzed, in particular, the definitions of the quark masses $m_{c}$ and $m_{b}$ in the two-loop matrix element of the four-quark operators $\mathcal{O}_{1,2}$ (see Fig. 22). Since the charm quarks in the matrix element $\left\langle\mathcal{O}_{1,2}\right\rangle$ are dominantly off shell, it can be argued that the running charm mass should be chosen instead of the pole mass. The latter choice was used in all previous analyses (Greub, Hurth, and Wyler, 1996a, 1996b; Chetyrkin et al., 1997; Ciuchini et al., 1998a; Greub and Hurth, 1999; Kagan and Neubert, 1999):

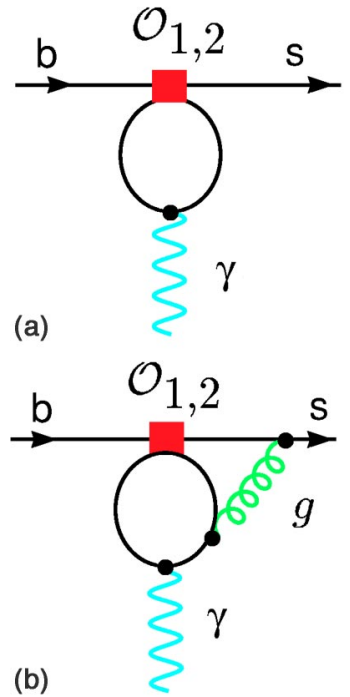

FIG. 22. (Color in online edition) The one-loop matrix element of the four-quark operators $\mathcal{O}_{1,2}$ vanishes (a), therefore the charm dependence starts with the two-loop (NLL) contribution (b).

$$
m_{c}^{\text {pole }} / m_{b}^{\text {pole }} \Rightarrow m_{c}^{\overline{M S}}(\mu) / m_{b}^{\text {pole }}, \quad \mu \in\left[m_{c}, m_{b}\right] .
$$

Numerically, the shift from $m_{c}^{\text {pole }} / m_{b}^{\text {pole }}=0.29 \pm 0.02$ to $m_{c}^{\overline{M S}}(\mu) / m_{b}^{\text {pole }}=0.22 \pm 0.04$ is rather important and leads to a $+11 \%$ shift of the central value of the $B$ $\rightarrow X_{s} \gamma$ branching ratio. The error in the charm mass within the $\overline{M S}$ scheme is due to the uncertainty resulting from the scale variation as well as to the uncertainty in $m_{c}^{\overline{M S}}\left(m_{c}^{\overline{M S}}\right)$. With their new choice of charm-mass renormalization scheme and with $\delta=0.9$, their theoretical prediction for the "total" branching ratio is ${ }^{3}$

$$
\mathcal{B}\left(B \rightarrow X_{s} \gamma\right)=(3.73 \pm 0.30) \times 10^{-4} \text {. }
$$

Since the matrix element starts at NLL order (see Fig. 22) and, thus, the renormalization scheme for $m_{c}$ is an NNLL issue, one should regard the choice of the $\overline{M S}$ scheme as an educated guess of the NNLL corrections. Nevertheless, the new choice is guided by the experience gained from many higher-order calculations in perturbation theory. Moreover, the $\overline{M S}$ mass of the charm quark is also a short-distance quantity which does not suffer from nonperturbative ambiguities, in contrast to its pole mass. Therefore the central value resulting within this scheme, Eq. (7.5), is definitely favored and should be regarded as the present theoretical prediction.

One should note that the scheme ambiguity regarding the $b$-quark mass is under control.

Because the choice of the renormalization scheme for $m_{c}$ is an NNLL effect, one has to emphasize that regarding the charm-mass scheme, the theoretical prediction (7.3) using the pole mass scheme is, in principle, as good

\footnotetext{
${ }^{3}$ Actually, the theoretical prediction of Gambino and Misiak (2001) is given for the energy cut $E \gamma=1.6 \mathrm{GeV}$ :

$$
\mathcal{B}\left(B \rightarrow X_{s} \gamma\right)_{E \gamma>1.6 \mathrm{GeV}}=(3.60 \pm 0.30) \times 10^{-4} \text {. }
$$
}

The theoretical error in Eq. (7.5) might be larger due to nonperturbative corrections (see Sec. III). 
as the new prediction (7.5) using the $\overline{M S}$ scheme; neither prediction takes into account the full impact of charm-mass-scheme ambiguity. Thus one has to argue for a larger theoretical uncertainty in $m_{c}^{\overline{M S}}(\mu) / m_{b}^{\text {pole }}$, which also includes the value of $m_{c}^{\text {pole }}$. This leads to a more appropriate error above $10 \%$ in Eq. (7.5).

One should emphasize that this present dominant uncertainty is due to a charm-mass-scheme ambiguity at the NLL level, i.e., to the question of whether the $\overline{M S}$ or the pole mass is more appropriate to estimate the unknown NNLL contributions. This uncertainty is of perturbative origin. The second uncertainty is due to the numerical value of $m_{c}$ within the specific choice of the charm-mass scheme and is a parametrical uncertainty. The Particle Data Review in its latest edition (Hagiwara et al., 2002) gives as the range for the latter uncertainty within the $\overline{M S}$ scheme $1.0 \mathrm{GeV} \leqslant m_{c}^{\overline{M S}}\left(m_{c}^{\overline{M S}}\right)$ $\leqslant 1.4 \mathrm{GeV}$ and has therefore doubled the uncertainty with respect to the one quoted previously (Groom et al., 2000). Nevertheless, there are determinations using the sum-rule technique (Eidemueller and Jamin, 2001; Kuhn and Steinhauser, 2001; Martin et al., 2001; Narison, 2001; Penarrocha and Schilcher, 2001) and also one that uses the lattice technique (in the quenched approximation; Rolf and Sint, 2002) which indicates much smaller uncertainties.

The only way to resolve the charm-mass-scheme ambiguity within the present theoretical NLL prediction and reduce the uncertainty below $10 \%$ would be an NNLL calculation, which is not beyond the power of present technical tools in perturbation theory. Such an ambitious program makes sense only if one is sure that the associated nonperturbative effects are under control. It was argued by Misiak (2002) that the numerical behavior of the branching ratio of $B \rightarrow X_{s} \gamma$ as a function of $m_{c}$ suggested that the dominant charm-mass dependence originates from distances much smaller than $\Lambda_{Q C D}$. In such a case, the associated nonperturbative effects would be under control and negligible.

\section{B. CKM phenomenology with $B \rightarrow X_{s, d} \gamma$}

Instead of making a theoretical prediction for the branching ratio $\mathcal{B}\left(B \rightarrow X_{s} \gamma\right)$, one can use the experimental data and theory in order to determine the combination $\left|V_{t b} V_{t s}^{*}\right| /\left|V_{c b}\right|$ of CKM matrix elements; in turn, one can determine $\left|V_{t s}\right|$ by making use of the relatively well-known CKM matrix elements $V_{c b}$ and $V_{t b}$. But when one has used the unitarity constraint in the theory prediction already, the $B \rightarrow X_{s} \gamma$ constraint on $V_{t s}$ does not add much to what is already known from the unitarity fit (Ali, 1997; Greub and Hurth, 1999). If one does not use the CKM unitarity, the sensitivity of $B$ $\rightarrow X_{s} \gamma$ to $V_{t s}$ becomes significantly reduced because the charm-quark contribution is twice as large as the topquark contribution.

A future measurement of the $B \rightarrow X_{d} \gamma$ decay rate will help to reduce the currently allowed region of the CKM Wolfenstein parameters $\rho$ and $\eta$. It is also of specific interest with respect to new physics, because its CKM suppression by the factor $\left|V_{t d}\right|^{2} /\left|V_{t s}\right|^{2}$ in the standard model may not be true in extended models.

Most of the theoretical improvements carried out in the context of the decay $B \rightarrow X_{s} \gamma$ can be straightforwardly adapted for the decay $B \rightarrow X_{d} \gamma$, and thus the NLL-improved and power-corrected decay rate for $B$ $\rightarrow X_{d} \gamma$ has much reduced theoretical uncertainty, which would allow an extraction of the CKM parameters from the measured branching ratio.

The predictions for the $B \rightarrow X_{d} \gamma$ decay given by Ali et al. (1998) show that, for the central values of the input parameters, the difference between the LL and NLL results is $\sim 10 \%$, increasing the branching ratio in the NLL case. Of particular theoretical interest is the ratio of the branching ratios, defined as

$$
R(d \gamma / s \gamma) \equiv \frac{\mathcal{B}\left(B \rightarrow X_{d} \gamma\right)}{\mathcal{B}\left(B \rightarrow X_{s} \gamma\right)},
$$

in which a good part of the theoretical uncertainties cancels. This suggests that a future measurement of $R(d \gamma / s \gamma)$ will have a large impact on the CKM phenomenology: varying the CKM Wolfenstein parameters $\rho$ and $\eta$ in the ranges $-0.1 \leqslant \rho \leqslant 0.4$ and $0.2 \leqslant \eta \leqslant 0.46$ and taking into account other parametric dependencies stated above, the results (without electroweak corrections) are

$$
\begin{aligned}
& 6.0 \times 10^{-6} \leqslant \mathcal{B}\left(B \rightarrow X_{d} \gamma\right) \leqslant 2.6 \times 10^{-5}, \\
& 0.017 \leqslant R(d \gamma / s \gamma) \leqslant 0.074
\end{aligned}
$$

In these predictions (Ali et al., 1998) it is assumed that the long-distance intermediate $u$-quark contributions play only a subdominant role (see the discussion at the end of Sec. VI).

As mentioned in Sec. III.B, the photon spectrum of $B \rightarrow X_{s} \gamma$ plays an important role in the determination of the CKM matrix element $V_{u b}$.

\section{Role of $b \rightarrow$ s gluon for $B \rightarrow X_{\text {no charm }}$}

Some remarks on the decay mode $b \rightarrow s$ gluon are in order. The effective Hamiltonian in the decay mode $b$ $\rightarrow s$ gluon coincides with the one in the decay $b \rightarrow s \gamma$. By replacing the photon by the gluon, one can use the NLL QCD calculation of $b \rightarrow s \gamma$. But in the calculation of the matrix element of the operator $\mathcal{O}_{2}$, further diagrams with the non-Abelian three-gluon coupling had to be calculated (Greub and Liniger, 2001). The NLL calculation (Greub and Liniger, 2001) leads to $\mathcal{B}(b$ $\rightarrow$ sluon $)=(5.0 \pm 1.0) \times 10^{-3}$, which is a factor of more than 2 larger than the former LL result $\mathcal{B}(b$ $\rightarrow$ s gluon $)=(2.2 \pm 0.8) \times 10^{-3} \quad$ (Ciuchini et al., 1993, 1994; Misiak, 1993; Cella et al., 1994). The mode b $\rightarrow$ sluon represents one component of the inclusive charmless hadronic decays, $B \rightarrow X_{\text {no charm, }}$ where $X_{n o}$ charm denotes any hadronic charmless final state. The corresponding branching ratio allows for the extraction of the ratio $\left|V_{u b} / V_{c b}\right|$ (Lenz et al., 1999). At the quark level, there are decay modes with three-body final 
$\frac{d}{d \hat{s}} B R\left(B \rightarrow X_{s} l^{+} l^{-}\right) \times 10^{-5}$

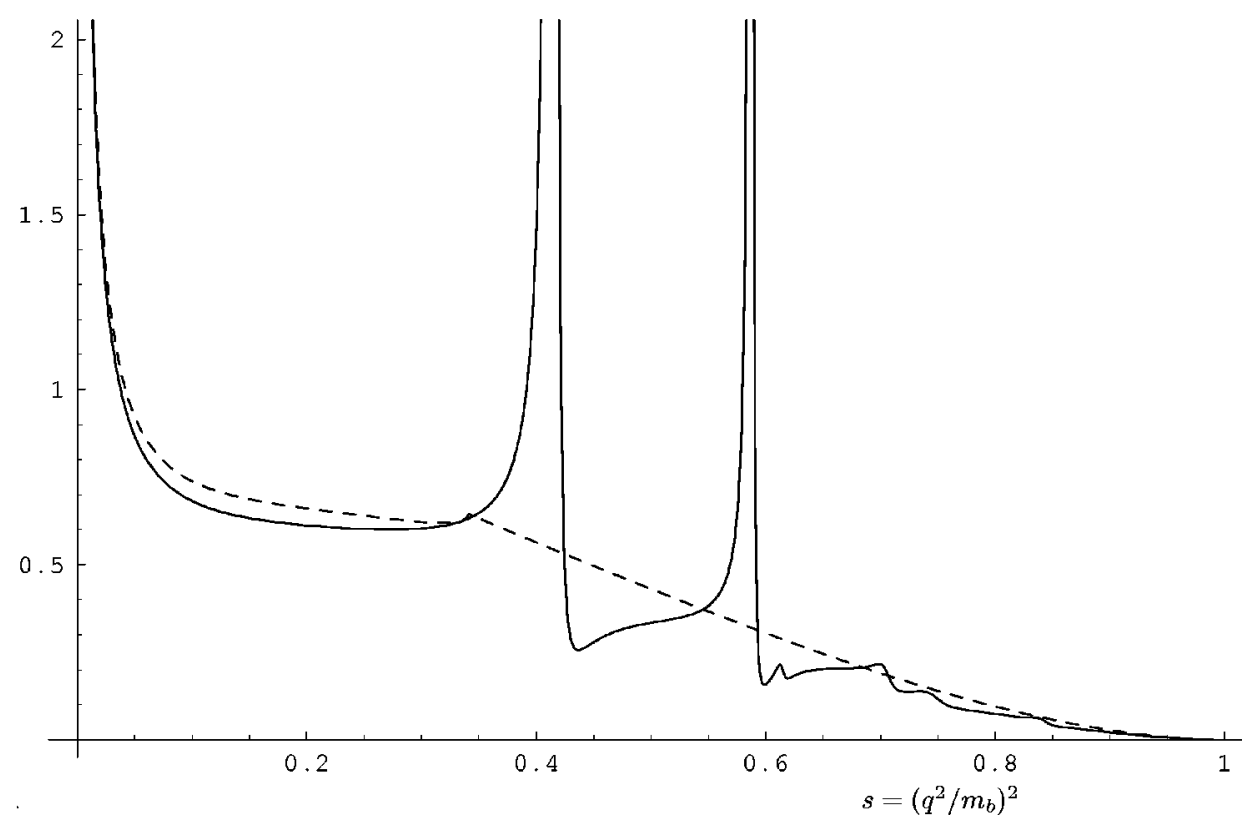

FIG. 23. Schematic dileptonmass spectrum of $B \rightarrow X_{s} \ell^{+} \ell^{-}$. The dashed line corresponds to the perturbative contribution. Courtesy of M. Misiak.

states, $b \rightarrow q^{\prime} \bar{q}^{\prime} q\left(q^{\prime}=u, d, s ; q=d, s\right)$ and the modes $b$ $\rightarrow q g$, with two-body final-state topology. The component $b \rightarrow s g$ of the charmless hadronic decays is expected to manifest itself in kaons with high momenta (of order $m_{b} / 2$ ), owing to its two-body nature (Kagan and Rathsman, 1997). The impact of the NLL corrections in $b$ $\rightarrow s g$ on the inclusive charmless hadronic decays, $B$ $\rightarrow X_{\text {no charm }}$, turns out to be as big as the NLL corrections to the $b$-quark decay modes with three quarks (Greub and Liniger, 2001).

There is still only marginal overlap between theory and experiment for the inclusive semileptonic branching ratio and the charm multiplicity in $B$-meson decays (Golutvin, 2001), if the usual scale variations are used in the theoretical predictions (Greub and Liniger, 2001). A possible reinforcement of the decay $b \rightarrow s g$ due to new physics through the chromomagnetic $\left(\mathcal{O}_{8}\right)$ contribution would lead to a natural explanation of these effects (Kagan and Rathsman, 1997). There is still room for such a scenario, which would also be compatible with the present $B \rightarrow X_{s} \gamma$ constraint (Besmer et al., 2001).

\section{Phenomenology of $B \rightarrow X_{s} \ell^{+} \ell^{-}$}

In comparison with $B \rightarrow X_{s} \gamma$, the inclusive $B$ $\rightarrow X_{s} \ell^{+} \ell^{-}$decay presents a complementary and also more complex test of the standard model. As mentioned above, this decay is also dominated by perturbative contributions if the $c \bar{c}$ resonances that show up as large peaks in the dilepton invariant-mass spectrum (see Fig. 23) are removed by appropriate kinematic cuts. In the "perturbative windows," that is, in the low- $\hat{s}$ region $0.05<\hat{s}=q^{2} / m_{b}^{2}<0.25$ as well as in the high- $\hat{s}$ region with $0.65<\hat{s}<\hat{s}_{\text {max }}$ (for $\hat{s}_{\text {max }}$ see Sec. VI), theoretical predictions for the invariant-mass spectrum are dominated by purely perturbative contributions, and a theoretical pre- cision comparable with the one reached in the decay $B$ $\rightarrow X_{s} \gamma$ is, in principle, possible. Regarding the choice of precise cuts in the dilepton-mass spectrum, it is important that one directly compare theory and experiment using the same energy cuts and avoid any kind of extrapolation.

In the high- $\hat{s}$ region, one should encounter the breakdown of the heavy-mass expansion at the end point (see Sec. VI). Integrated quantities are still defined; nevertheless one finds sizable $\Lambda_{Q C D}^{2} / m_{b}^{2}$ nonperturbative corrections within this region. Rather large Wilson coefficients to order $\Lambda_{Q C D}^{3} / m_{b}^{3}$ were found by Bauer and Burrell (2000). These coefficients can be used to give an estimate of these corrections while the corresponding matrix elements are unknown. Following an argument of Neubert (2000), one can show that this $\Lambda_{Q C D} / m_{b}$ expansion is effectively a $\Lambda_{Q C D} / m_{c}$ one in the high- $\hat{s}$ region.

The kinematic observables, the invariant dileptonmass spectrum, and the forward-backward asymmetry are usually normalized by the semileptonic decay rate in order to reduce the uncertainties due to bottom-quark mass and CKM angles. The partonic dilepton invariantmass spectrum and the forward-backward asymmetry are defined as

$$
R_{\text {quark }}^{\ell^{+} \ell^{-}}(\hat{s})=\frac{d}{d \hat{s}} \Gamma\left(b \rightarrow X_{s} \ell^{+} \ell^{-}\right) / \Gamma\left(b \rightarrow X_{c} e \bar{\nu}\right)
$$

$$
\begin{aligned}
A_{F B}(\hat{s})= & \frac{1}{\Gamma\left(b \rightarrow X_{c} e \bar{\nu}\right)} \\
& \times \int_{-1}^{1} d \cos \theta_{\ell} \frac{d^{2} \Gamma\left(b \rightarrow X_{s} \ell^{+} \ell^{-}\right)}{d \hat{s} d \cos \theta_{\ell}} \operatorname{sgn}\left(\cos \theta_{\ell}\right),
\end{aligned}
$$


where $\theta_{\ell}$ is the angle between $\ell^{+}$and $B$ momenta in the dilepton center-of-mass frame. ${ }^{4}$

It was shown by Ali et al. (1997) that $A_{F B}(\hat{s})$ is equivalent to the energy asymmetry introduced by Cho et al. (1996):

$$
A_{\text {energy }}=\frac{N\left(E_{\ell^{-}}>E_{\ell^{+}}\right)-N\left(E_{\ell^{+}}>E_{\ell^{-}}\right)}{N\left(E_{\ell^{-}}>E_{\ell^{+}}\right)+N\left(E_{\ell^{+}}>E_{\ell^{-}}\right)},
$$

where $N\left(E_{\ell^{-}}>E_{\ell^{+}}\right)$denotes the number of lepton pairs whose negatively charged member is more energetic in the $B$-meson rest frame than its positive partner. From the experimental point of view, there is no significant difference either between measuring the inherent asymmetry using the lepton energy distribution or using the three-momentum information.

For the low- $\hat{s}$ region the present partonic NNLL prediction is given by (Asatryan et al., 2002; Ghinculov et al., 2003b):

$$
\int_{0.05}^{0.25} d \hat{s} R_{\text {quark }}^{\ell^{+} \ell^{-}}(\hat{s})=\left(1.27 \pm 0.08_{\text {scale }}\right) \times 10^{-5}
$$

The error quoted in Eq. (7.10) reflects only the renormalization scale uncertainty and is purely perturbative. There is no additional problem due to the charm-mass renormalization-scheme ambiguity within the decay $B$ $\rightarrow X_{s} \ell^{+} \ell^{-}$because the charm dependence starts already at one loop, in contrast to the case of the decay $B$ $\rightarrow X_{s} \gamma$ (see Fig. 16). The charm dependence itself leads to an additional uncertainty of $\sim 7.6 \%$ within the partonic quantity (7.10), if the pole mass is varied, $m_{c}^{\text {pole }} / m_{b}^{\text {pole }}=0.29 \pm 0.02$.

As discussed in Sec. V, the impact of the NNLL contributions is significant. The large matching scale $\mu_{W}$ uncertainty of $16 \%$ of the NLL result was removed; the low-scale uncertainty $\mu_{b}$ of $13 \%$ was cut in half; in addition the central value of the integrated low dilepton spectrum (7.10) was significantly changed by $\approx-14 \%$ due to NNLL corrections.

These small uncertainties in the inclusive mode should be compared with those of the corresponding exclusive mode $B \rightarrow K^{*} \mu^{+} \mu^{-}$given by Ali et al. (2000): $\Delta \mathcal{B}$ $=\left({ }_{-17}^{+26}, \pm 6,{ }_{-4}^{+6},{ }_{+0.4}^{-0.7}, \pm 2\right) \%$. The first dominant error represents the hadronic uncertainty due to the form factors.

Using the measured semileptonic branching ratio $\mathcal{B}_{\text {exp }}^{s l}$, the prediction for the corresponding branching ratio is given by

\footnotetext{
${ }^{4}$ The so-called "normalized" forward-backward asymmetry, which is also often used, is given by

$$
\begin{aligned}
\bar{A}_{F B}(\hat{s})= & \int_{-1}^{1} d \cos \theta_{\ell}\left[d^{2} \Gamma\left(B X_{s} \ell^{+} \ell^{-}\right) / d \hat{s} d \cos \theta_{\ell}\right] \operatorname{sgn}\left(\cos \theta_{\ell}\right) / \\
& \int_{-1}^{1} d \cos \theta_{\ell}\left[d^{2} \Gamma\left(B \rightarrow X_{s} \ell^{+} \ell^{-}\right) / d \hat{s} d \cos \theta_{\ell}\right]
\end{aligned}
$$
}

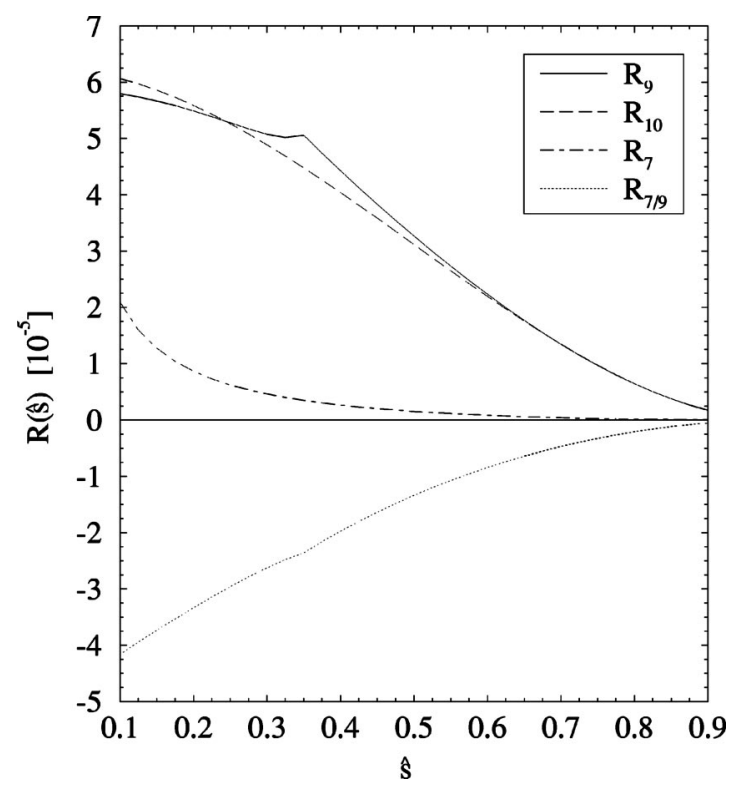

FIG. 24. Comparison of the different short-distance contributions to $R_{\text {quark }}^{\ell^{+} \ell^{-}}(\hat{s})$ (NLL precision). From Buras and Munz, 1995.

$$
\begin{aligned}
\mathcal{B}(B & \left.\rightarrow X_{s} \ell^{+} \ell^{-}\right)_{\text {cut }} \hat{s} \in[0.05,0.25] \\
& =\mathcal{B}_{\exp }^{s l} \int_{0.05}^{0.25} d \hat{s}\left[R_{\text {quark }}^{\ell^{+} \ell^{-}}(\hat{s})+\delta_{1 / m_{b}^{2}} R(\hat{s})+\delta_{1 / m_{c}^{2}} R(\hat{s})\right] \\
& =0.104\left[\left(1.27 \pm 0.08_{\text {scale }}\right)+0.06-0.02\right] \times 10^{-5} \\
& =\left(1.36 \pm 0.08_{\text {scale }}\right) \times 10^{-6} .
\end{aligned}
$$

Here $\delta_{1 / m_{b}^{2}} R(\hat{s})$ and $\delta_{1 / m_{c}^{2}} R(\hat{s})$ are the nonperturbative contributions discussed in Sec. VI. The recent first measurement of BELLE, with a rather large uncertainty (Kaneko et al., 2003), is compatible with this standardmodel prediction.

One might think that, within the perturbative window at low $\hat{s} \in[0.05,0.25]$, the sensitivity is only to $C_{7}$, which would be redundant information, since we already know this from the decay $B \rightarrow X_{s} \gamma$. But, as was explicitly shown by Misiak (1993) and Buras and Munz (1995), there is also sensitivity to the new Wilson coefficients $C_{9}$ and $C_{10}$ and interference terms in the low- $\hat{s}$ regime with $\hat{s} \in[0.05,0.25]$ [see Fig. 24, where the various perturbative contributions to $R_{\text {quark }}^{\ell^{+} \ell^{-}}$(with NLL precision) are plotted].

A phenomenological NNLL analysis including the high-dilepton-mass region is presented by Ghinculov et al. (2003).

The phenomenological impact of the NNLL contributions on the forward-backward asymmetry is also significant (Asatrian et al., 2002; Ghinculov et al., 2003a). The position of the zero of the forward-backward asymmetry, defined by $A_{F B}\left(\hat{s}_{0}\right)=0$, is particularly interesting in determining the relative sign and magnitude of the Wilson coefficients $C_{7}$ and $C_{9}$, and it is therefore extremely sensitive to possible new physics effects.

Employing the counting rule proposed by Asatryan et al. (2001), i.e., treating the formally $O\left(1 / \alpha_{s}\right)$ term of 
$C_{9}$ as $O(1)$ (see discussion at the end of Sec. V), one can determine the lowest-order value of $\hat{s}_{0}$-formally derived by the NLL expression of $A_{F B}$-by the solution of

$$
\hat{s}_{0} C_{9}^{\mathrm{eff}}\left(\hat{s}_{0}\right)+2 C_{7}^{\mathrm{eff}}=0,
$$

where the effective coefficients $C_{i}^{\text {eff }}$ also encode all dominant matrix-element corrections, which leads to an explicit $\hat{s}$ dependence [see Ghinculov et al. (2003a), Eq. (A.1)]. One arrives at

$$
\hat{s}_{0}^{N L L}=0.14 \pm 0.02 \text {, }
$$

where the error is determined by the scale dependence. That NLL result is now modified by the NNLL contributions (Asatrian et al., 2002; Ghinculov et al., 2003a) to

$$
\hat{s}_{0}^{N N L L}=0.162 \pm 0.008 .
$$

In this case the variation of the result induced by the scale dependence is accidentally very small (about $\pm 1 \%$ ) and cannot be regarded as a good estimate of missing higher-order effects. Taking into account the separate scale variation of both Wilson coefficients $C_{9}$ and $C_{7}$ and the charm-mass dependence, one estimates a conservative overall error on $\hat{s}_{0}$ of about $5 \%$ (Ghinculov et al., 2003a). In this $\hat{s}$ region the nonperturbative $1 / m_{b}^{2}$ and $1 / m_{c}^{2}$ corrections to $A_{F B}$ are very small and also under control (see Sec. VI).

An illustration of the shift of the central value and the reduced scale dependence between NNL and NNLL expressions of $A_{F B}(s)$, in the low- $\hat{s}$ region, is presented in Fig. 25. The complete effect of NNLL contributions to the forward-backward asymmetry adds up to a $16 \%$ shift compared with the NLL result, with a residual error due to higher-order terms reduced at the 5\% level. Thus the zero of the forward-backward asymmetry in the inclusive mode turns out to be one of the most sensitive tests for new physics beyond the standard model.

The $B$ factories will soon provide statistics and the resolution needed for the measurements of $B$ $\rightarrow X_{s} \ell^{+} \ell^{-}$kinematic distributions. Precise theoretical estimates of the standard-model expectations are therefore needed in order to perform significant new tests of flavor physics. The recently calculated new (NNLL) contributions (Bobeth et al., 2000b; Asatryan et al., 2001, 2002; Ghinculov et al., 2003a, 2003b, 2003c) have significantly improved the sensitivity of the inclusive $B$ $\rightarrow X_{s} \ell^{+} \ell^{-}$decay in testing extensions of the standard model in the sector of flavor dynamics. Together with the decay $B \rightarrow X_{s} \gamma$, the inclusive $B \rightarrow X_{s} \ell^{+} \ell^{-}$decay will make precision flavor physics possible.

\section{E. Golden mode $B \rightarrow X_{s} \bar{\nu} \nu$}

The decay $B \rightarrow X_{s} \nu \bar{\nu}$ is the theoretically cleanest rare $B$ decay, but also the most difficult experimentally.

As discussed in Sec. V, the dependence of the partonic decay rate on the up-quark and charm-quark masses is negligible. The perturbative scale uncertainty is $O(1 \%)$. Moreover, the nonperturbative contributions

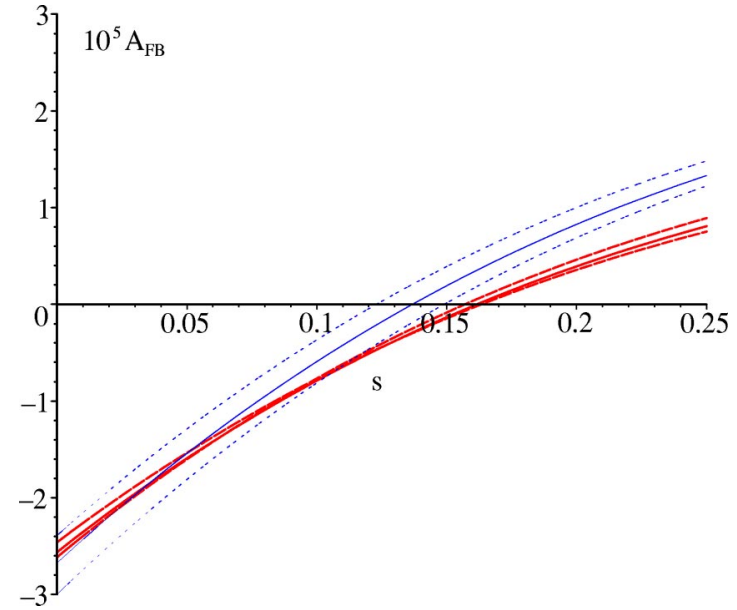

FIG. 25. (Color in online edition) Comparison between NNLL and NLL results for $A_{F B}(\hat{s})$ in the low- $\hat{s}$ region. The three thick lines are the NNLL predictions for $\mu=5 \mathrm{GeV}$ (full), and $\mu=2.5$ and $10 \mathrm{GeV}$ (dashed); the dotted curves are the corresponding NLL results. All curves are for $m_{c} / m_{b}=0.29$. Note that $A_{F B}(s)$ is normalized by the semileptonic decay rate. See Eq. (7.8).

scaling with $1 / m_{b}^{2}$ are under control and small (Falk et al., 1994; Ali et al., 1997; Buchalla and Isidori, 1998). Because of the absence of the photon penguin contribution, the nonperturbative contributions scaling with $1 / \mathrm{m}_{c}^{2}$ can be estimated to be at the level of $10^{-3}$ at most (Buchalla et al., 1998).

After normalizing to the semileptonic branching ratio and summing over the three neutrino flavors, one obtains the branching ratio of the decay $B \rightarrow X_{s} \nu \bar{\nu}$ given by (Buchalla and Buras, 1993; Buras, 1998)

$$
\begin{aligned}
\mathcal{B}(B & \left.\rightarrow X_{s} \nu \bar{\nu}\right) \\
& =\mathcal{B}_{\exp }^{s l} \frac{12 \alpha^{2}}{\pi^{2} \sin ^{4} \Theta_{W}} \frac{\left|V_{t s}\right|^{2}}{\left|V_{c b}\right|^{2}} \frac{C\left(m_{t}^{2} / m_{W}^{2}\right) \bar{\eta}}{f\left(m_{c}^{2} / m_{b}^{2}\right) \kappa\left(m_{c}^{2} / m_{b}^{2}\right)} .
\end{aligned}
$$

Using the measured semileptonic branching ratio and the phase-space factor of the semileptonic decay $f$, the corresponding QCD correction $\kappa$, the QCD correction of the matrix element of the decay $B \rightarrow X_{s} \nu \bar{\nu}$, namely, $\bar{\eta}=\kappa(0)$, and scanning the input parameters, one ends up with a theoretical prediction that includes a very conservative error estimate (Buras, 1998):

$$
\mathcal{B}\left(B \rightarrow X_{s} \nu \bar{\nu}\right)=(3.5 \pm 0.7) \times 10^{-5} \text {. }
$$

The replacement of $V_{t s}$ by $V_{t d}$ in Eq. (7.15) leads to the case of the decay $B \rightarrow X_{d} \nu \bar{\nu}$. Obviously all uncertainties cancel out in the ratio of the two branching ratios of $B \rightarrow X_{d} \nu \bar{\nu}$ and $B \rightarrow X_{s} \nu \bar{\nu}$. It thus allows for the cleanest direct determination of the ratio of the two corresponding CKM matrix elements.

The measurement of these decay modes is rather difficult. The neutrinos escape detection; one thus has to search for the decays $B \rightarrow X_{s, d} \nu \bar{\nu}$ through large missing energy associated with the two neutrinos. Clearly, background control is more than difficult in these channels. 
Hopefully, the $B$ factories, with their high statistics and their clean environment, will be able to measure these decays in the future.

However, the lack of an excess of events with large missing energy in a sample of $0.5 \times 10^{6} b \bar{b}$ pairs at LEP has already allowed ALEPH to establish an upper bound on the branching ratio of $B \rightarrow X_{s} \nu \bar{\nu}$ (ALEPH, 1996; Grossman et al., 1996) at 90\% C.L.,

$$
\mathcal{B}\left(B \rightarrow X_{s} \nu \bar{\nu}\right)<7.7 \times 10^{-4} .
$$

This upper bound is still an order of magnitude above the standard-model prediction, but it already leads to constraints on new-physics models (Grossman et al., 1996). For this purpose, the QCD corrections to the decays $B \rightarrow X_{s, d} \nu \bar{\nu}$ in supersymmetric theories have recently been presented (Bobeth et al., 2002).

\section{INDIRECT SEARCH FOR SUPERSYMMETRY}

\section{A. Generalities}

Today supersymmetric models are given priority in our search for new physics beyond the standard model. This is primarily suggested by theoretical arguments related to the well-known hierarchy problem. Supersymmetry eliminates the sensitivity for the highest scale in the theory, and thus stabilizes the low-energy theory. The precise mechanism of the necessary supersymmetry breaking is unknown. A reasonable approach to this problem is the inclusion of the most general soft breaking terms consistent with the standard-model gauge symmetries in the so-called unconstrained minimal supersymmetric standard model (MSSM). This leads to a proliferation of free parameters in the theory.

In the MSSM there are new sources of FCNC transitions. Besides the CKM-induced contributions, which are brought about by a charged Higgs or a chargino, there are generic supersymmetric contributions that arise from flavor mixing in the squark-mass matrices. The structure of the MSSM does not explain the suppression of FCNC processes, which is observed in experiments; the gauge symmetry within the supersymmetric framework does not protect the observed strong suppression of the FCNC transitions. This is the crucial point of the well-known supersymmetric flavor problem. Clearly, the origin of flavor violation is highly model dependent.

Within the framework of the MSSM there are at present three favored supersymmetric models that solve the supersymmetric flavor problem by a specific mechanism through which the sector of supersymmetry breaking communicates with the sector accessible to experiments: in the minimal-supergravity (mSUGRA) model (Barbieri et al., 1982; Chamseddine et al., 1982; Hall et al., 1983), supergravity is the corresponding mediator; in the other two models, this is achieved by gauge interactions (GMSB) (Dimopoulos and Raby, 1981; Dine et al., 1981, 1995, 1996; Dine and Nelson, 1993) and by anomalies (AMSB) (Giudice et al., 1998; Randall and Sundrum, 1999). Furthermore, there are other classes of models in which the flavor problem is solved by particular flavor symmetries (Dine et al., 1993; Nir and Seiberg, 1993; Leurer et al., 1994; Dimopoulos and Giudice, 1995; Barbieri et al., 1996; Cohen et al., 1996; Pomarol and Tommasini, 1996).

The decay $B \rightarrow X_{s} \gamma$ is sensitive to the mechanism of supersymmetry breaking because, in the limit of exact supersymmetry, the decay rate would be just zero:

$$
\mathcal{B}\left(B \rightarrow X_{\text {s }} \gamma\right)_{\text {exact susy }}=0 .
$$

This follows from an argument first given in 1974 (Ferrara and Remiddi, 1974). In that work the absence of the anomalous magnetic moment in a supersymmetric Abelian gauge theory was shown.

Flavor violation thus originates from the interplay between the dynamics of flavor and the mechanism of supersymmetry breaking. FCNC processes therefore yield important (indirect) information on the construction of supersymmetric extensions of the standard model and can contribute to the question of which mechanism ultimately breaks supersymmetry. In this context it is important to analyze the correlations between the different sets of information from rare $B$ and $K$ decays. Moreover, tight experimental bounds on some flavor-diagonal transitions, such as the electric dipole moment of the electron and of the neutron, as well as $g$-2, help to constrain the soft terms that induce chirality violations.

As was already emphasized in the Introduction, inclusive rare decays, as loop-induced processes, are particularly sensitive to new physics and theoretically clean. Neutral flavor transitions involving third-generation quarks, typically in the $B$ system, do not yet pose serious threats to specific models, except for the rare $B \rightarrow X_{s} \gamma$ decay, which has already carved out large regions in the space of free parameters of most of the models in the classes mentioned above. Once more precise data from the $B$ factories are available, this decay will undoubtedly gain even more efficiency for selecting viable regions of the parameter space in the various classes of models. This indirect search for new physics is a modeldependent issue, especially in the MSSM with its 43 new $C P$-violating phases. Simplifying assumptions about the parameters often introduce model-dependent correlations between different observables. Thus flavor physics will also help to discriminate between the models that will be proposed by then. In view of this, it is important to calculate the rate of the rare $B$ decays, with theoretical uncertainties as reduced as possible and general enough for generic supersymmetric models.

In the analysis of FCNC processes within supersymmetry, the additional assumption of minimal flavor violation (MFV) is often introduced. Minimal flavor violation is then loosely defined as "the flavor violation that is completely dictated by the CKM angles." In a top/ bottom approach, one starts with a specific model of supersymmetry breaking and then tries to justify the simplifying assumption of MFV explicitly within the specific model. In a bottom/top approach, the naive assumption of MFV is problematic, since it is not stable, within the MSSM, under radiative corrections and calls for a more precise concept. D'Ambrosio et al. (2002) present a con- 
sistent definition, which essentially requires that all flavor- and $C P$-violating interactions be linked to the known structure of Yukawa couplings. The constraint within an effective-field approach is introduced with the help of a symmetry concept and can be shown to be renormalization-group invariant; it is also a valid concept beyond supersymmetric models (D'Ambrosio et al., 2002). This consistent MFV assumption, for example, is valid if the soft terms of the scalar mass are universal and the trilinear soft terms are proportional to Yukawa couplings at an arbitrary high scale. Then the physical squark masses are not equal, but the induced flavor violation is described in terms of the usual CKM parameters.

Perhaps this MFV-based, effective-field-theory approach is too pessimistic from the current point of view. One of the key predictions of the MFV is the direct link between the $b \rightarrow s, b \rightarrow d$, and $s \rightarrow d$ transitions. This prediction within the $\Delta F=1$ sector is definitely not well tested at the moment.

In contrast to the scale of electroweak symmetry breaking, there is no similarly strong argument that new flavor structures have to appear at the electroweak scale.

\section{B. Constraints from $B \rightarrow X_{s} \gamma$}

While in the standard model, the rate for $B \rightarrow X_{s} \gamma$ is known up to NLL in QCD, the calculation of this decay rate within supersymmetric models is still far from this level of sophistication. There are several contributions to the decay amplitude: besides the $W t$-quark and the $H t$-quark contributions, there are also the chargino, gluino, and neutralino contributions. The first systematic MSSM analysis of the decay $B \rightarrow X_{s} \gamma$ was presented by Bertolini et al. (1991).

The phenomenological analyses of the decay $B$ $\rightarrow X_{s} \gamma$ in the mSUGRA model (Borzumati, 1994; Baer et al., 1998; Goto et al., 1998) have already excluded large parts of the parameter space of this model. However, within many analyses the nonstandard contributions were often not investigated with NLL precision as was the standard-model contribution. Besides the large uncertainties in the LL predictions, the step from LL to NLL precision is also necessary in order to check the validity of the perturbative approach in the model under consideration. Moreover, it has already been shown in specific new-physics scenarios that bounds on the parameter space of nonstandard models are very sensitive to NLL contributions (see below).

Nevertheless, within supersymmetric models, partial NLL results are available. The gluonic NLL two-loop matching contributions were presented some time ago (Bobeth et al., 2000a). A complete NLL calculation of the $B \rightarrow X_{s} \gamma$ branching ratio in the simplest extension of the standard model, namely, the two-Higgs-doublet model, is already available (Borzumati and Greub, 1998; Ciuchini et al., 1998a; Gambino and Misiak, 2001): in the two-Higgs-doublet model of type II (which already represents a good approximation for gauge-mediated supersymmetric models with large $\tan \beta$, where the charged

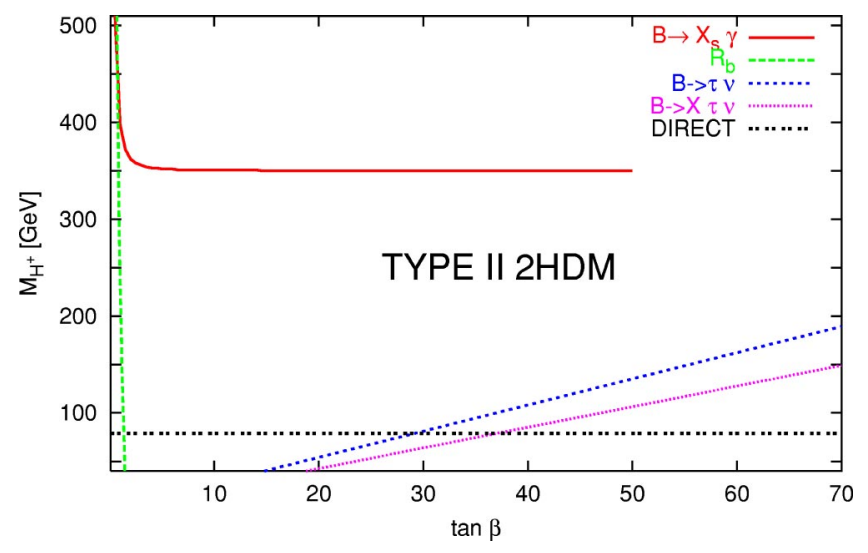

FIG. 26. (Color in online edition) Direct and indirect lower bounds on $M_{H^{+}}$from different processes in the two-Higgsdoublet model of type II as a function of $\tan \beta$. The $B \rightarrow X_{s} \gamma$ bound is based on the latest CLEO measurement [Eq. (3.2)]. From Gambino and Misiak, 2001.

Higgs contribution dominates the chargino contribution), the $B \rightarrow X_{s} \gamma$ is only sensitive to two parameters of this model, the mass of the charged Higgs boson and $\tan \beta$. Thus the experimental data of the decay $B$ $\rightarrow X_{s} \gamma$ allow for stringent bounds on these two parameters, which are much more restrictive than the lower bound on the charged Higgs mass found in the direct search at LEP (see Fig. 26).

One finds that these indirect bounds are very sensitive to NLL QCD corrections and even to the two-loop electroweak contributions (see Borzumati and Greub, 1998; Ciuchini et al., 1998a). Using the latest theoretical NLL prediction (7.5) and the latest CLEO measurement (3.2), one finds the $\tan \beta$ independent bound $M_{H}$ $>350 \mathrm{GeV}$ (Gambino and Misiak, 2001). But this bound gets weakened if the charm-mass renormalizationscheme ambiguity of the present NLL prediction (see Sec. VII.A) is taken into account. For example, if the pole-mass scheme is adapted as in the theoretical prediction (8.3), then the weaker bound $M_{H}>280 \mathrm{GeV}$ is found (Gambino and Misiak, 2001).

Ciuchini et al. (1998b) present a specific supersymmetric scenario in which the possibility of destructive interference of the chargino and the charged Higgs contribution are particularly analyzed. The analysis has been done under two assumptions. The first is that the only source of flavor violation at the electroweak scale is that of the standard model, encoded in the CKM matrix (MFV). Therefore the analysis applies to mSUGRA, GMSB, and AMSB models (in which the same features are assumed at the messenger scale) only when the sources of flavor violation, generated radiatively between the supersymmetry-breaking scale, and the electroweak scale, can be neglected with respect to those induced by the CKM matrix. The second assumption is that there exists a specific mass hierarchy, in particular, the heavy-gluino limit. Indeed, the NLL calculation has been done in the limit

$$
\begin{aligned}
\mu_{\tilde{g}} & \sim O\left(m_{\tilde{g}}, m_{\bar{q}}, m_{\tilde{t}_{1}}\right) \gg \mu_{W} \\
& \sim O\left(m_{W}, m_{H^{+}}, m_{t}, m_{\chi}, m_{\tilde{t}_{2}}\right) .
\end{aligned}
$$




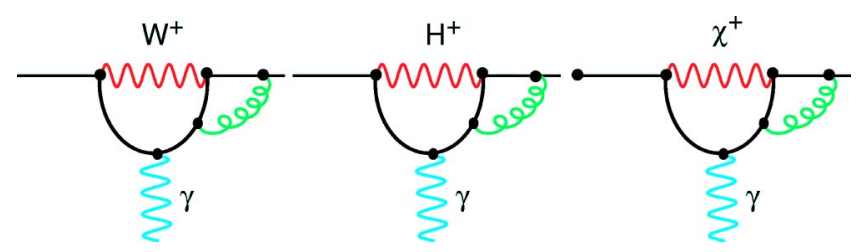

FIG. 27. (Color in online edition) Standard-model, charged Higgs, and chargino contributions at the matching scale.

The mass scale of the charginos $(\chi)$ and of the lighter stop $\left(\tilde{t}_{2}\right)$ is the ordinary electroweak scale $\mu_{W}$, while the scale $\mu_{g}$ is characteristic of all other strongly interacting supersymmetric particles (squarks and gluinos) and is assumed to be of the order of $1 \mathrm{TeV}$. Next-toleading-logarithm QCD corrections have been calculated up to first order in $\mu_{W} / \mu_{\tilde{g}}$, including the important nondecoupling effects (Ciuchini et al., 1998b).

At the electroweak scale $\mu_{W}$, the new contributions do not induce any new operators in this scenario. Thus the only step in the new NLL calculation beyond the one within the standard model is Step 1, the matching calculation at the scale $\mu_{W}$, where we encounter the two new CKM-induced contributions of the charged Higgs and the chargino (see Fig. 27):

$$
C_{N L L}\left(\mu_{W}\right)=C_{N L L}^{S M}\left(\mu_{W}\right)+C_{N L L}^{H^{+}}\left(\mu_{W}\right)+C_{N L L}^{\chi}\left(\mu_{W}\right) .
$$

It was found (Ciuchini et al., 1998b) that, in this specific supersymmetric scenario, bounds on the parameter space are rather sensitive to NLL contributions, and they lead to a significant reduction of the stop-chargino mass region, where the supersymmetric contribution has a large destructive interference with the charged-Higgsboson contribution. In Fig. 28 the upper bounds on the lighter chargino and stop masses from $B \rightarrow X_{s} \gamma$ data in the scenario of Eq. (8.2) are illustrated if a light charged Higgs mass of $m_{H^{ \pm}}=100 \mathrm{GeV}$ is assumed. The stop mixing is set to $\left|\theta_{t}^{-}\right|<\pi / 10$, which corresponds to the assumption of a mainly right-handed light stop. Moreover, $|\mu|<500 \mathrm{GeV}$ and all heavy masses are around $1 \mathrm{TeV}$. For $\tan \beta=2$ and 4 the results of the LL, "NLL running," and NLL calculations are given. The result of neglecting the new NLL supersymmetric contributions to the Wilson coefficients is labeled as "NLL running" and illustrates the importance of the NLL chargino contribution (Ciuchini et al., 1998b).

This specific MFV scenario was refined and extended to the large $\tan \beta$ regime by the resummations of terms of the form $\alpha_{s}^{n} \tan ^{n+1} \beta$ (Degrassi et al., 2000; Carena et al., 2001). Additional $\tan \beta$ terms, which have to be summed in the large $\tan \beta$ regime, were singled out by D'Ambrosio et al. (2002). The stability of the renormalization-group-improved perturbation theory was reassured for this specific scenario: the resummed NLL results in the large $\tan \beta$ regime show constraints similar to the LL results (see de Boer et al., 2001). For example, it is a well-known feature in the mSUGRA model that, depending on the sign of $A_{t} \cdot \mu$ (where $A_{t}$ denotes the stop-mixing parameter and $\mu$ the Higgsino

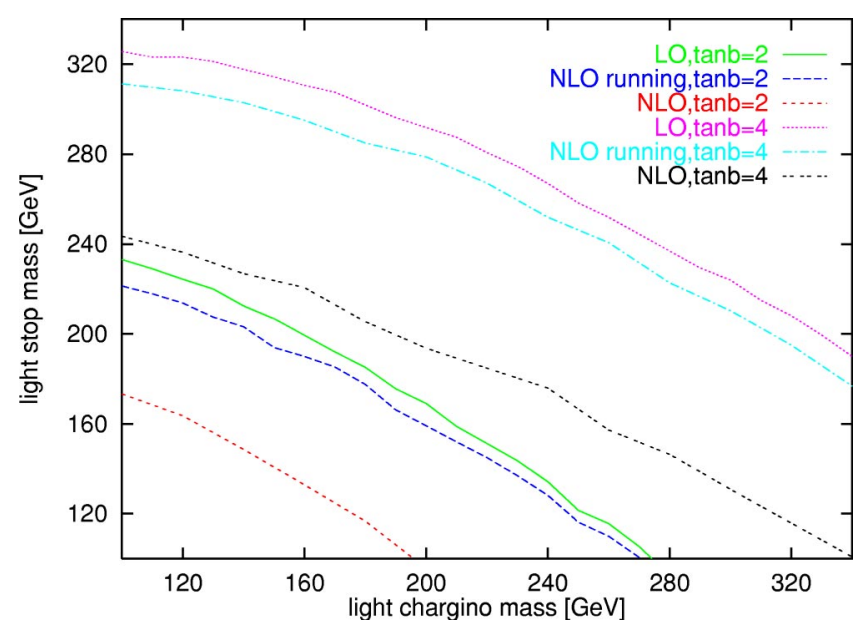

FIG. 28. (Color in online edition) Upper bounds on the lighter chargino and stop masses from $B \rightarrow X_{s} \gamma$ data in the scenario of Eq. (8.2) if a light charged Higgs mass is assumed; for $\tan \beta$ $=2$ (three lower curves) and 4 (three upper plots) the LL, NLL-running, and NLL results (from the top to the bottom) are shown (see text). From Ciuchini et al., 1998b.

mass parameter), the chargino contribution can interfere constructively $\left(A_{t} \cdot \mu>0\right)$ or destructively $\left(A_{t} \cdot \mu<0\right)$ with the standard model and the charged Higgs contribution. Therefore the scenario $A_{t} \cdot \mu>0$ within this model requires very heavy superpartners in order to accommodate the $B \rightarrow X_{s} \gamma$ data. But the case $A_{t} \cdot \mu<0$ is also constrained in the large $\tan \beta$ regime where the chargino contribution is strongly enhanced (Degrassi et al., 2000; Carena et al., 2001; de Boer et al., 2001; see Fig. 29).

However, all these NLL analyses are valid only in the heavy-gluino regime. Thus they cannot be used in particular directions of the parameter space of the abovelisted models in which quantum effects induce a gluino contribution as large as the chargino or the standardmodel contributions. Nor can it be used as a modeldiscriminator tool, to constrain the potentially large sources of flavor violation typical of generic supersymmetric models. Therefore a complete NLL calculation, also within the MFV approach, should include contributions in which the gluon is replaced by its superpartner gluino (see Fig. 30; Greub et al., 2003).

The flavor-nondiagonal gluino-quark-squark vertex induced by the flavor-violating scalar mass term and trilinear terms is particularly interesting. This vertex is generically assumed to induce the dominant contribution to quark flavor transitions, since this vertex is weighted by the strong-coupling constant $g_{s}$. Therefore it is often taken as the only contribution to these transitions and, in particular, to the $B \rightarrow X_{s} \gamma$ decay when attempting to obtain order-of-magnitude upper bounds on flavorviolating terms in the scalar potential. Once the experimental constraints are imposed, however, the gluino contribution is reduced to values such that the standardmodel and the other supersymmetric contributions can no longer be neglected. Any LL and NLL calculation of 

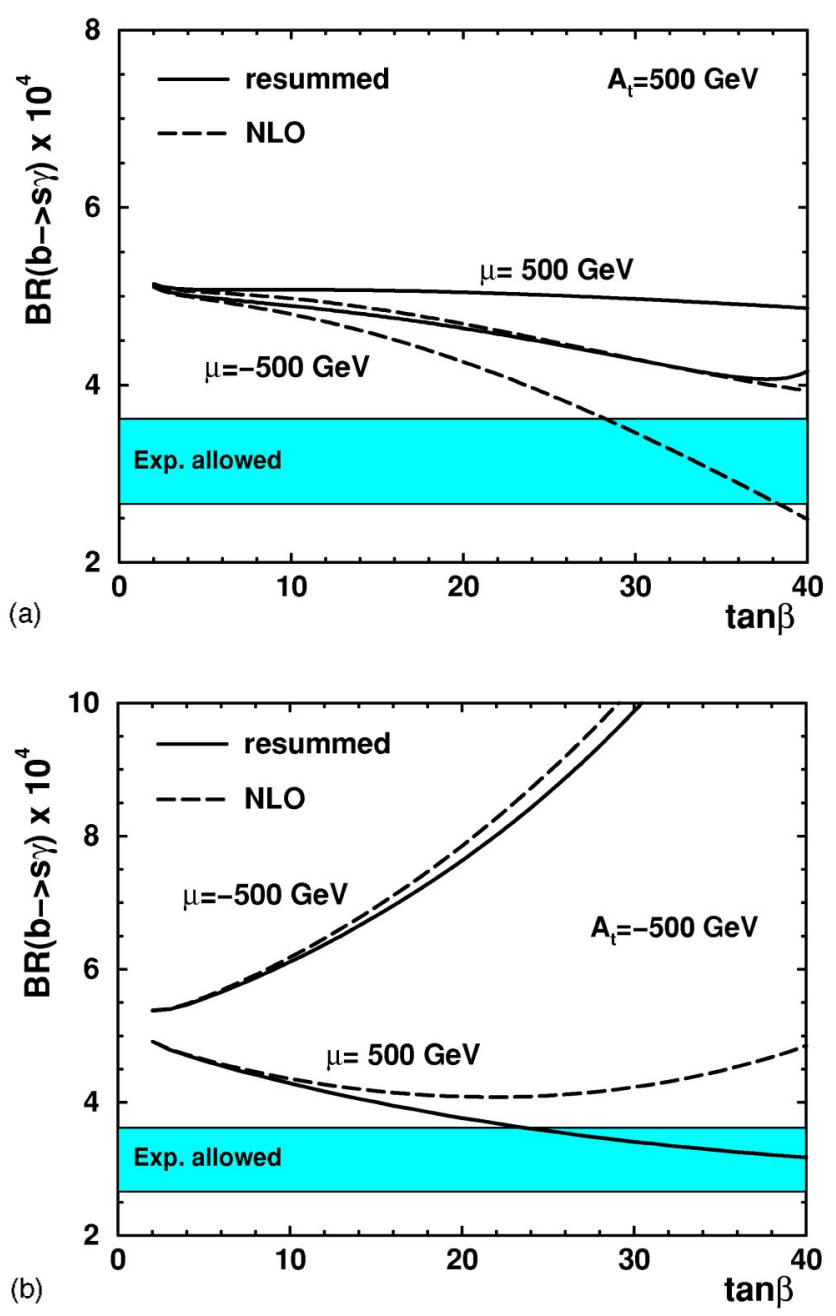

FIG. 29. (Color in online edition) Comparison of the theoretical NLL predictions within a special minimal supersymmetric standard model (MSSM) scenario [similiar to Eq. (8.2)] with and without the resummed large $\tan \beta$ terms; the chargedHiggs-boson mass is $200 \mathrm{GeV}$ and the light stop mass is 250 $\mathrm{GeV}$. The values of $\mu$ and $A_{t}$ are indicated in the plot, while the gluino, heavy stop, and down-squark masses are set at 800 GeV. From Carena et al., 2001.

the $B \rightarrow X_{s} \gamma$ rate in generic supersymmetric models should therefore include all possible contributions.

The gluino contribution presents some peculiar features related to the implementation of the QCD corrections. Borzumati et al. (2000) investigated this contribution to the decay $B \rightarrow X_{s} \gamma$ in great detail for supersymmetric models with generic soft terms. The gluino-induced contributions to the decay amplitude for $B \rightarrow X_{s} \gamma$ are of the form

$$
\begin{array}{ll}
\alpha_{s}\left(m_{b}\right)\left[\alpha_{s}\left(m_{b}\right) \ln \left(m_{b} / M\right)\right]^{n} & (\mathrm{LL}), \\
\alpha_{s}^{2}\left(m_{b}\right)\left[\alpha_{s}\left(m_{b}\right) \ln \left(m_{b} / M\right)\right]^{n} & (\mathrm{NLL}) .
\end{array}
$$

The relevant operator basis of the standard-model effective Hamiltonian gets enlarged to contain magnetic and chromomagnetic operators with an extra factor of $\alpha_{s}$. Furthermore, one finds that gluino-squark boxes induce new scalar and tensorial four-quark operators, which are

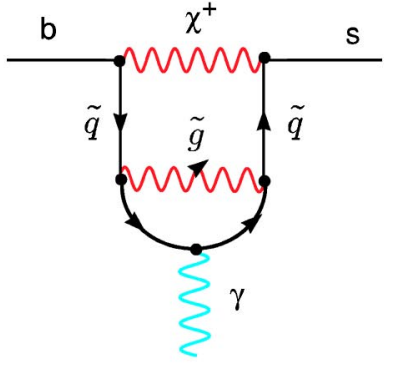

(a)

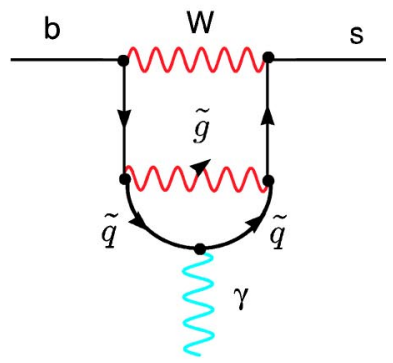

(b)

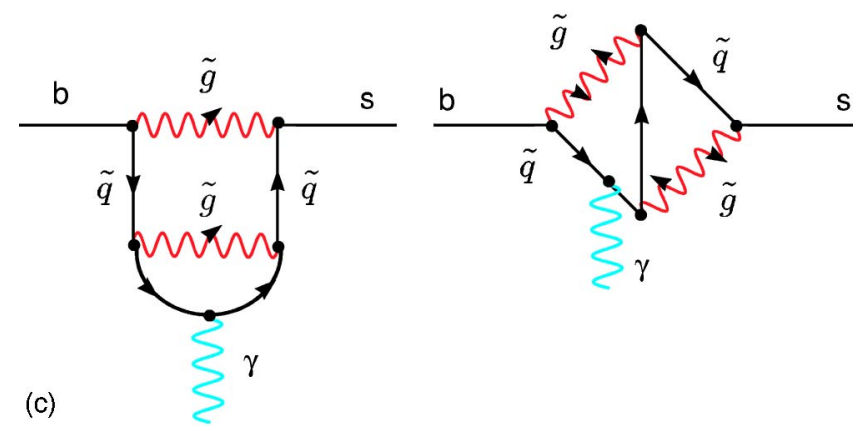

FIG. 30. (Color in online edition) Some of the NLL gluino contributions to the decay $B \rightarrow X_{s} \gamma$.

shown to mix into the magnetic operators without gluons already at one loop. On the other hand, the vectorial four-quark operators mix only with an additional gluon into magnetic ones (Fig. 31). Thus they will contribute at NLL order only. But from the numerical point of view the contributions of the vectorial operators (although NLL) are not necessarily suppressed with respect to the new four-quark contributions; the flavor-violation parameters present in the Wilson coefficients of the new operators are expected to be much smaller (or much more stringently constrained) than the corresponding ones in the coefficients of the vectorial operators. This feature shows that a complete NLL calculation is important.

To understand the sources of flavor violation that may be present in supersymmetric models in addition to those enclosed in the CKM matrix, one has to consider the contributions to the squark mass matrices,

$\mathcal{M}_{f}^{2} \equiv\left[\begin{array}{cc}m_{f, L L}^{2}+F_{f L L}+D_{f L L} & \left(m_{f, L R}^{2}\right)+F_{f L R} \\ \left(m_{f, L R}^{2}\right)^{\dagger}+F_{f R L} & m_{f, R R}^{2}+F_{f R R}+D_{f R R}\end{array}\right]$,

where $f$ stands for up- or down-type squarks. In the super-CKM basis, where the quark-mass matrices are diagonal and the squarks are rotated in parallel to their superpartners, the $F$ terms from the superpotential and 


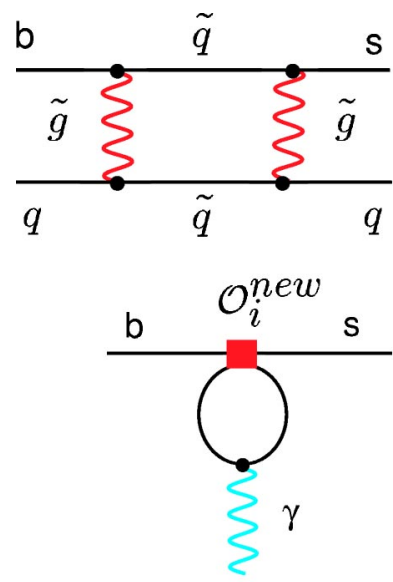

(a)
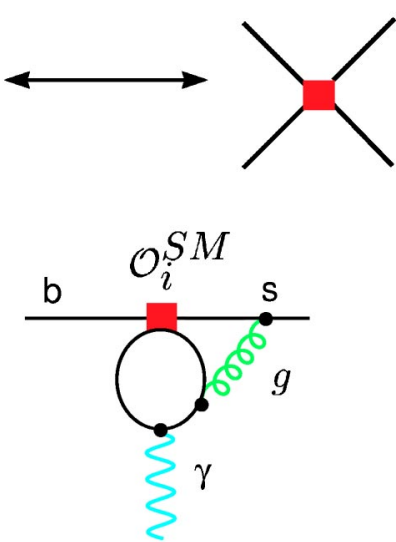

(b)
FIG. 31. (Color in online edition) New scalar operators. Upper figure: matching of gluino-squark box on new scalar operators; lower figure: mixing of new (scalar) operators at one loop (a) in contrast to the vectorial operators of the standard model (b) which mix at two loops only.

the $D$ terms turn out to be diagonal $3 \times 3$ submatrices of the $6 \times 6$ mass matrices $\mathcal{M}_{f}^{2}$. This is in general not true for the additional terms $m_{f}^{2}$, originating from the soft supersymmetric-breaking potential. Because all neutral gaugino couplings are flavor diagonal in the super-CKM basis, the gluino contributions to the decay $b \rightarrow s \gamma$ are induced by the off-diagonal elements of the soft terms $m_{f, L L}^{2}, m_{f, R R}^{2}$, and $m_{f, R L}^{2}=\left(m_{f, L R}^{2}\right)^{\dagger}$.

As a first step, it is convenient to select one possible source of flavor violation in the squark sector at a time and assume that all the remaining ones are vanishing. Following Hall et al. (1986) and Gabbiani et al. (1996), one sets all diagonal entries in $m_{d, L L}^{2}, m_{d, R R}^{2}$, and $m_{u, R R}^{2}$ to be equal and their common value to be denoted by $m_{\tilde{q}}^{2}$. The branching ratio can then be studied as a function of

$$
\begin{aligned}
& \delta_{L L, i j}=\frac{\left(m_{d, L L}^{2}\right)_{i j}}{m_{\tilde{q}}^{2}}, \quad \delta_{R R, i j}=\frac{\left(m_{d, R R}^{2}\right)_{i j}}{m_{\tilde{q}}^{2}}, \\
& \delta_{L R, i j}=\frac{\left(m_{d, L R}^{2}\right)_{i j}}{m_{\tilde{q}}^{2}} \quad(i \neq j) .
\end{aligned}
$$

Phenomenological analyses in the so-called unconstrained MSSM (Donoghue et al., 1983; Hagelin et al., 1994; Gabbiani et al., 1996) neglected QCD corrections and only used the gluino contribution to saturate the experimental bounds. Moreover, no correlations between different sources of flavor violation were taken into account. In this way, one arrived at "order-ofmagnitude bounds" on the soft parameters (Hagelin et al., 1994; Gabbiani et al., 1996). The $B \rightarrow X_{s} \gamma$ decay is mainly sensitive to the off-diagonal elements $\delta_{L R, 23}$ and $\delta_{R L, 23}$ and constrains them to values of order $10^{-2}$. Borzumati et al. (2000) systematically analyzed the sensitivity of the bounds on the down-squark mass matrix to radiative QCD LL corrections, including the standard- model and gluino contributions. Some leading NLL contributions were considered by Okumura and Roszkowski (2002) and the large impact of the NLL corrections for nonminimal models, in particular, for large $\tan \beta$ was demonstrated.

A consistent analysis of the bounds on the sfermion mass matrix should also include interference effects between the various contributions. Besmer et al. (2001) analyzed the interplay between the various sources of flavor violation and the interference effects of standardmodel, gluino, chargino, neutralino, and charged-Higgsboson contributions. New bounds on simple combinations of elements of the soft part of the squark mass matrices were found to be, in general, one order of magnitude weaker than the bound on the single off-diagonal element $\delta_{L R, 23}$, which was derived in previous work (Gabbiani et al., 1996; Masiero and Vives, 2001) by neglecting any kind of interference effects. Thus it turns out that - at least within the decay $B \rightarrow X_{s} \gamma$-the flavor problem is less severe than often stated.

The measurement of the photon polarization within the decay $B \rightarrow X_{s} \gamma$ allows for another important test of the standard model. Assuming that the decay is induced, by the magnetic dipole operator only, one starts with the effective Hamiltonian

$$
\mathcal{H}=-\frac{4 G_{F}}{\sqrt{2}} \lambda_{t}\left(C_{7 L} \mathcal{O}_{7 L}+C_{7 R} \mathcal{O}_{7 R}\right),
$$

where $\mathcal{O}_{7 L, R} \equiv\left(e / 16 \pi^{2}\right) \bar{s} \sigma_{\mu \nu}\left(1 \pm \gamma_{5} / 2\right) b F^{\mu \nu}$. Then the photon polarization is defined by

$$
\lambda_{\gamma} \equiv \frac{\left|C_{7 R}\right|^{2}-\left|C_{7 L}\right|^{2}}{\left|C_{7 R}\right|^{2}+\left|C_{7 L}\right|^{2}} .
$$

In the standard model, one has $C_{7 R} / C_{7 L}=m_{s} / m_{b} \approx 0$ and therefore a mostly left-handed photon. But in many supersymmetric scenarios, and also in left-rightsymmetric models, the photon may have a large righthanded component. Everett et al. (2002) discussed the possibility of a strictly right-handed photon within the framework of the MSSM. Clearly, only in nonminimal models is such an extreme deviation from the standardmodel prediction possible.

There are many suggestions for measuring $\lambda_{\gamma}$ in the literature (Atwood et al., 1997; Mannel and Recksiegel, 1997; Melikhov et al., 1998; Grossman and Pirjol, 2000; Kim et al., 2000; Kruger et al., 2000; Hiller and Kagan, 2002). However, they all rely on very high statistics or on new experimental settings and will not be possible in the near future. Quite recently, a new method has been proposed, which can be realized with the present statistics available at the $B$ factories. The photon polarization can be measured in radiative $B$ decays to excited kaons, using angular correlations among the three-body decay products of the excited kaons (Gronau and Pirjol, 2002; Gronau et al., 2002). It is essential for a helicity measurement to have a three-particle decay mode because $\lambda_{\gamma}$ is a parity-odd variable and there is no oddmomentum correlation in the two-body mode. In the decays $B^{+} \rightarrow\left[K_{1}^{+}(1400) \rightarrow K^{0} \pi^{+} \pi^{0}\right] \gamma$ and $B^{0}$ 
$\rightarrow\left[K_{1}^{0}(1400) \rightarrow K^{+} \pi^{-} \pi^{0}\right] \gamma$, however, the up-down asymmetry of the photon momentum with respect to the $K \pi \pi$ decay plane in the frame of the excited kaon measures the photon polarization rather efficiently. The updown asymmetry can theoretically be predicted to be $A=(0.33 \pm 0.05) \times \lambda_{\gamma}$ in the case of the resonance $K_{1}$ (1400) (Gronau and Pirjol, 2002; Gronau et al., 2002). Thus the method will definitely be sensitive to large deviations from the standard-model prediction even with the present luminosity at the $B$ factories.

\section{Constraints from $B \rightarrow X_{s} \ell^{+} \ell^{-}$}

The inclusive $B \rightarrow X_{s} \ell^{+} \ell^{-}$decay is another important tool for understanding the nature of physics beyond the standard model. In comparison to the decay $B \rightarrow X_{s} \gamma$, it offers complementary information. For example, one is able to resolve the sign ambiguity of the Wilson coefficient $C_{7}$, which is not fixed by the $B \rightarrow X_{s} \gamma$ constraint. The forward-backward asymmetry, however, has terms proportional to $\operatorname{Re}\left(C_{10} C_{9}^{\text {eff }}\right)$ and $\operatorname{Re}\left(C_{10} C_{7}^{\text {eff }}\right)$. The invariant dilepton mass spectrum, the forward-backward charge asymmetry, and the decay rate of $B \rightarrow X_{s} \gamma$ determine the magnitude and also the sign of the three Wilson coefficients $C_{7}, C_{9}$, and $C_{10}$, and allow for a model-independent analysis of rare $B$ decays as was first advocated by Ali et al. (1995).

There are several mSUGRA models as well as several model-independent analyses in the literature (Bertolini et al., 1991; Cho et al., 1996; Goto et al., 1997; Hewett and Wells, 1997; Huang et al., 1999; Kim et al., 1999; Lunghi et al., 2000). It was always assumed that the operator basis is not enlarged in comparison to the standard model. All the analyses found strong correlations between the decays $B \rightarrow X_{s} \gamma$ and $B \rightarrow X_{s} \ell^{+} \ell^{-}$.

Within the mSUGRA model, sizable deviations from the standard model values of the $B \rightarrow X_{s} \ell^{+} \ell^{-}$decay are excluded through the severe constraints on $C_{7}$ by the $B \rightarrow X_{s} \gamma$ measurement. But it was also shown that, in less restricted scenarios, supersymmetric contributions could potentially enhance the $B \rightarrow X_{s} \ell^{+} \ell^{-}$kinematic distributions, the dilepton mass spectrum, and the forward-backward asymmetry, by more than $100 \%$ relative to the standard model predictions. One of the reasons for enhancements is that the Wilson coefficient $C_{7}$ can change sign with respect to the standard-model in some region of the parameter space. As Fig. 32 shows, the non-standard-model sign of $C_{7}$ is not yet ruled out within the MFV scenario.

When the experimental uncertainties are reduced soon, this fact will permit discrimination between MFV models and nonminimal models and will lead either to evidence of new physics or to very stringent constraints on the parameter space of such models.

Recently these analyses have been updated by Ali et al. (2002) based on the new experimental data of the semileptonic decays and on partial results in the NNLL theoretical predictions. Within the analysis, the charmmass renormalization-scheme ambiguity (see Sec. VII.A) in the decay $B \rightarrow X_{s} \gamma$ is taken into account. But a
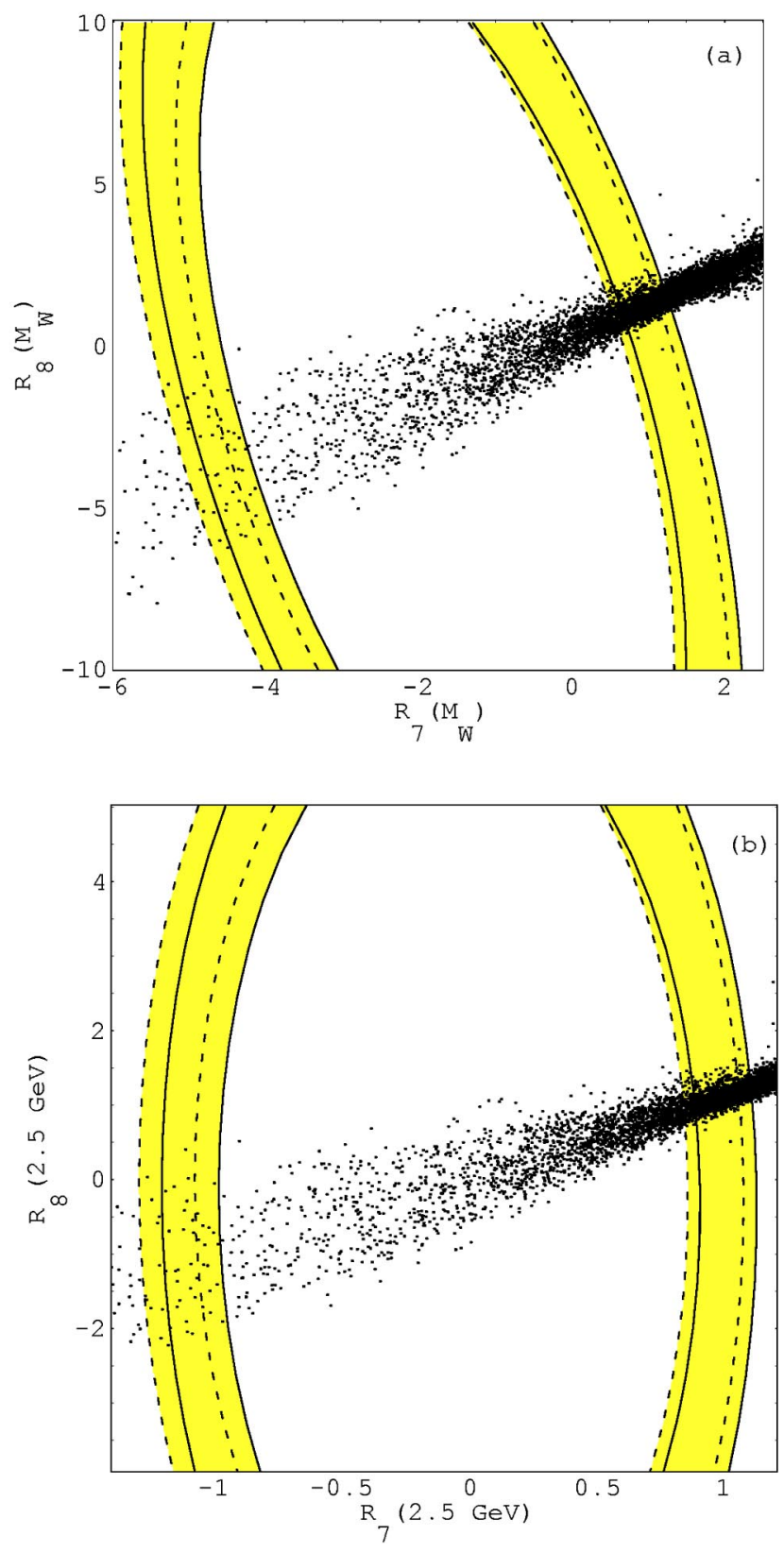

FIG. 32. (Color in online edition) $90 \%$ C.L. bounds in the $\left[R_{7}(\mu), R_{8}(\mu)\right]$ plane following from the measurement of the $B \rightarrow X_{s} \gamma$ branching ratio for $\mu=m_{W}$ (a) and $\mu=2.5 \mathrm{GeV}$ (b), where $R_{7,8}=C_{7,8}^{\text {total }} / C_{7,8}^{S M}$. Theoretical uncertainties are taken into account. The solid and dashed lines correspond to the $m_{c}=m_{c}^{\text {pole }}$ and $m_{c}=m_{c}^{\overline{M S}}\left(\mu_{b}\right)$ cases, respectively. The scatter points correspond to the expectation in minimal flavor violation (MFV) models. From Ali et al., 2002.

too-conservative error estimate regarding the charmmass dependence within the decay $B \rightarrow X_{s} \ell^{+} \ell^{-}$is also assumed, which leads to a rather large error of $15 \%$ in the inclusive mode.

It was found (Ali et al., 2002) that, with the present experimental knowledge, the decay $B \rightarrow X_{s} \gamma$ still leads to the most restrictive constraints. The MFV scenarios especially are already highly constrained, and only small deviations from the standard-model rates and distribu- 


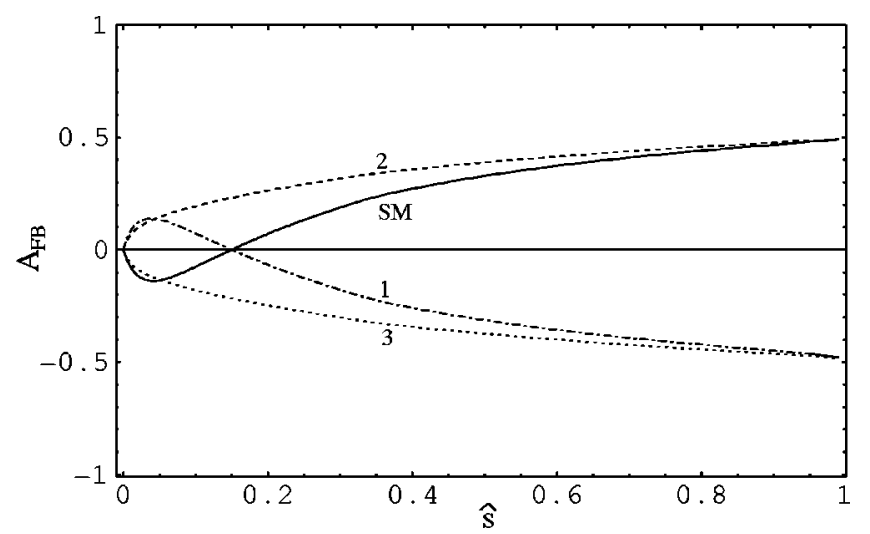

FIG. 33. Four different shapes of the normalized forwardbackward asymmetry $\bar{A}_{F B}$ for the decay $B \rightarrow X_{s} \ell^{+} \ell^{-}$. The four curves correspond to four sample points of the Wilson coefficients that are compatible with the present measurements of the integrated branching ratios. From Ali et al., 2002.

tions are possible; therefore no useful additional bounds from the semileptonic modes beyond those already known from the $B \rightarrow X_{s} \gamma$ decay can be deduced for the MFV models at the moment. But in nonminimal models, additional constraints from the semileptonic mode are already emerging in some parts of the supersymmetric parameter space, namely, for the off-diagonal elements within the squark mass matrix in the up-quark sector.

Within the model-independent analysis, the impact of the partial NNLL contributions on the allowed ranges for the Wilson coefficients has already been found to be significant. In this analysis, however, only integrated branching ratios were used to derive constraints. It is clear that one needs measurements of the kinematic distributions of the $B \rightarrow X_{s} \ell^{+} \ell^{-}$, the dilepton mass spectrum, and the forward-backward asymmetry in order to determine the exact values and signs of the Wilson coefficients. In Fig. 33, the impact of these future measurements is illustrated. It shows the shape of the forwardbackward asymmetry for the standard model and three additional sample points, which are all still allowed by the present measurements of the branching ratios. Thus even rather rough measurements of the forwardbackward asymmetry will either rule out large parts of the parameter space of extended models or show clear evidence for new physics beyond the standard model.

\section{DIRECT CP VIOLATION IN $b \rightarrow s$ TRANSITIONS}

The $B$ system provides us with an independent test of the CKM prescription of $C P$ violation. Until recently, the neutral-kaon system was the only environment in which $C P$ violation had been observed. Those effects in the kaon system are often plagued by large theoretical uncertainties due to long-range QCD. So it was difficult to decide whether the CKM description really accounted quantitatively for $C P$ violation. In contrast, nonperturbative contributions are under control in the $B$ system thanks to the heavy-mass expansion. Moreover, there are gold-plated $C P$ asymmetries such as the one in the decay mode $B \rightarrow \psi K_{S}$, which are theoretically very clean, because the direct decay amplitude is dominated by one single weak phase and, thus, most of the hadronic uncertainties drop out in the $C P$ asymmetry.

The CKM prescription of $C P$ violation with one single phase-proposed in 1972 when the second family was not confirmed experimentally (Kobayashi and Maskawa, 1973) - is very predictive and has now passed its first precision test in the golden $B$ mode, $B_{d}$ $\rightarrow \psi K_{S}$, at the $10 \%$ level (Abe et al., 2002b; Aubert et al., 2002b). Nevertheless, there is still room for nonstandard $C P$ phases, especially in the FCNC $\Delta F=1$ modes. Actually, detailed measurements of $C P$ asymmetries in rare $B$ decays will be possible in the near future.

The direct normalized $C P$ asymmetries of the inclusive decay modes are given by ${ }^{5}$

$$
\alpha_{C P}\left(B \rightarrow X_{s / d} \gamma\right)=\frac{\Gamma\left(\bar{B} \rightarrow X_{s / d} \gamma\right)-\Gamma\left(B \rightarrow X_{\bar{s} / \bar{d}} \gamma\right)}{\Gamma\left(\bar{B} \rightarrow X_{s / d} \gamma\right)+\Gamma\left(B \rightarrow X_{\bar{s} / d} \gamma\right)} .
$$

CLEO has already presented a measurement of the $C P$ asymmetry in the inclusive decay $B \rightarrow X_{s} \gamma$, which is actually a measurement of a weighted sum, $\alpha_{C P}$ $=0.965 \alpha_{C P}\left(B \rightarrow X_{s} \gamma\right)+0.02 \alpha_{C P}\left(B \rightarrow X_{d} \gamma\right), \quad$ yielding (Coan et al., 2001)

$$
\alpha_{C P}=(-0.079 \pm 0.108 \pm 0.022) \times(1.0 \pm 0.030) .
$$

The first error is statistical, and the second and third errors additive and multiplicative systematic, respectively. This measurement is based on $10^{7} B \bar{B}$ events and implies that, at a $90 \%$ confidence level, $\alpha_{C P}$ lies in the range $-0.27<\alpha_{C P}<+0.10$; very large effects are thus already excluded. The same conclusion can be deduced from the measurements of the $C P$ asymmetry in the exclusive mode $B \rightarrow K^{*}(892) \gamma$ of CLEO (Coan et al., 2000), $\quad \alpha_{C P}=+0.08 \pm 0.13_{\text {stat }} \pm 0.03_{\text {syst }}, \quad$ of BABAR (Aubert et al., 2002a), $\alpha_{C P}=-0.044 \pm 0.076 \pm 0.082$, and of BELLE (Abe et al., 2002c), $\alpha_{C P}=-0.022 \pm 0.048$ \pm 0.017 . The preliminary measurement of BELLE is the best by far, based on $65.4 \times 10^{6} \mathrm{~B}$-meson pairs, and implies that, at a $90 \%$ confidence level, $\alpha_{C P}$ in the exclusive $B \rightarrow K^{*} \gamma$ lies in the range $-0.106<\alpha_{C P}<+0.062$.

Theoretical next-to-leading-logarithm QCD predictions of the normalized CP asymmetries of the inclusive channels (see Ali et al., 1998 and Kagan and Neubert, 1998) within the standard model can be expressed by the approximate formulas (Kiers et al., 2000)

$$
\begin{aligned}
& \alpha_{C P}\left(B \rightarrow X_{s} \gamma\right) \approx 0.334 \times \operatorname{Im}\left[\epsilon_{s}\right] \approx+0.6 \%, \\
& \alpha_{C P}\left(B \rightarrow X_{d} \gamma\right) \approx 0.334 \times \operatorname{Im}\left[\epsilon_{d}\right] \approx-16 \%,
\end{aligned}
$$

where

${ }^{5}$ There is a sign convention that is generally adopted in theory and experiment: on the partonic level $\alpha_{C P}(b \rightarrow s \gamma)$ $=[\Gamma(b \rightarrow s \gamma)-\Gamma(\bar{b} \rightarrow \bar{s} \gamma)] /[\Gamma(b \rightarrow s \gamma)+\Gamma(\bar{b} \rightarrow \bar{s} \gamma)] ; \quad$ analogously $\alpha_{C P} \sim\left[\Gamma\left(\bar{B}^{0} \rightarrow \cdots\right)-\Gamma\left(B^{0} \rightarrow \cdots\right)\right]$ and $\alpha_{C P} \sim\left[\Gamma\left(B^{-}\right.\right.$ $\left.\rightarrow \cdots)-\Gamma\left(B^{+} \rightarrow \cdots\right)\right]$. 


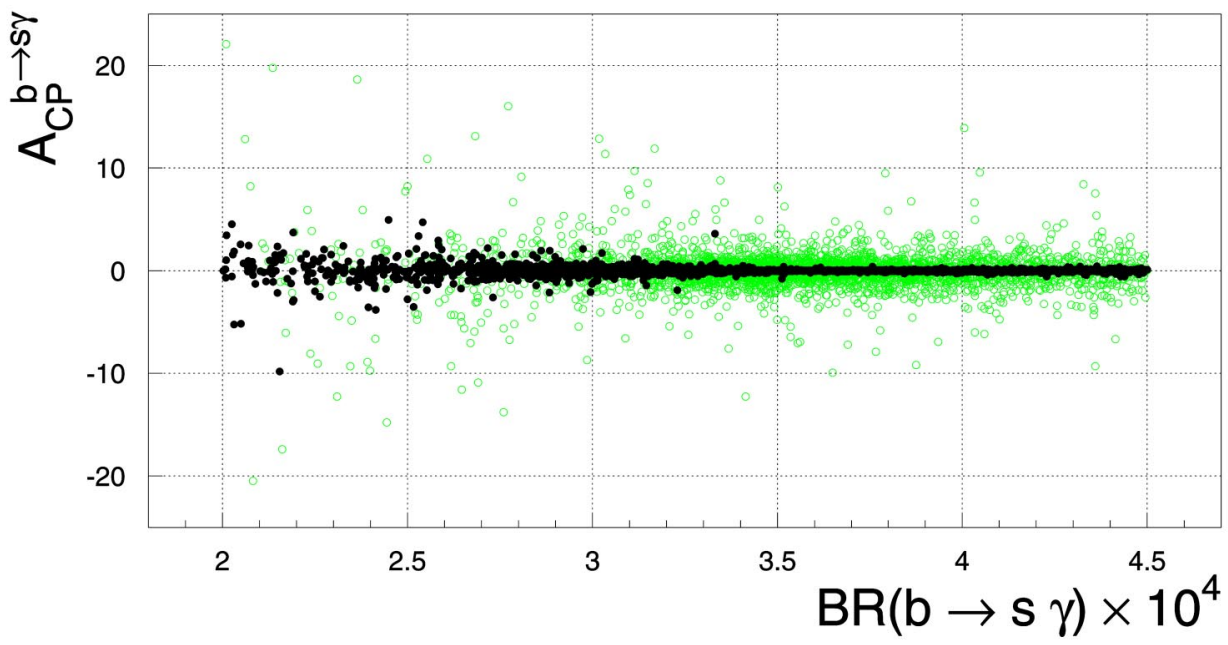

FIG. 34. (Color in online edition) $C P$ asymmetry vs "total" width of the decay $B \rightarrow X_{s} \gamma: \bigcirc$, computed without any restriction on the phases; $\boldsymbol{O}$, showing the impact of the EDM's constraints. From Bartl et al., 2001.

$$
\begin{gathered}
\epsilon_{s}=\frac{V_{u s}^{*} V_{u b}}{V_{t s}^{*} V_{t b}} \simeq-\lambda^{2}(\rho-i \eta), \\
\epsilon_{d}=\frac{V_{u d}^{*} V_{u b}}{V_{t d}^{*} V_{t b}} \simeq \frac{\rho-i \eta}{1-\rho+i \eta} .
\end{gathered}
$$

Numerically, the best-fit values of the CKM parameters are used (Kiers et al., 2000). The two $C P$ asymmetries are connected by the relative factor $\lambda^{2}\left[(1-\rho)^{2}+\eta^{2}\right]$. Moreover, the small standard-model prediction of $C P$ asymmetry in the decay $B \rightarrow X_{s} \gamma$ is a result of three suppression factors. There is an $\alpha_{s}$ factor needed in order to have a strong phase; moreover, there is a CKM suppression of order $\lambda^{2}$, and there is a GIM suppression of order $\left(m_{c} / m_{b}\right)^{2}$ reflecting the fact that in the limit $m_{c}$ $=m_{u}$ any $C P$ asymmetry in the standard model would vanish.

An analysis of the leptonic counterparts is presented by Ali and Hiller (1999). The normalized $C P$ asymmetries may also be calculated for exclusive decays: a model calculation has been offered by Greub et al. (1995). Theoretical predictions based on the QCD factorization approach (Beneke, Feldmann, and Seidel, 2001; Ali and Parkhomenko, 2002; Bosch and Buchalla, 2002) are also affected by large uncertainties. Only in the case of relatively large new-physics effects will one be able to disentangle these effects from the QCD uncertainties. But the available experimental data do not support this scenario in the $B \rightarrow K^{*} \gamma$ mode (Coan et al., 2000; Abe et al., 2002c; Aubert et al., 2002a).

Supersymmetric predictions for the $C P$ asymmetries in $B \rightarrow X_{s / d} \gamma$ depend strongly on what is assumed for the supersymmetry-breaking sector and are thus a rather model-dependent issue. The minimal-supergravity model cannot account for large $C P$ asymmetries beyond $2 \%$ because of the constraints coming from the electron and neutron electric dipole moments (Goto et al., 1999). This is generally true in models based on the MFV assumption (see also Fig. 34). Nonminimal models with squark mixing or models with $R$-parity violation allow for asymmetries, of the order of $10 \%$ or even larger $(\mathrm{Ka}-$ gan and Neubert, 1998; Aoki et al., 1999; Baek and Ko,
1999; Chua et al., 1999; Giusti et al., 1999; Kim et al., 1999; Chun et al., 2000; Bailin and Khalil, 2001; Demir and Olive, 2002) argue that the asymmetry in nonminimal models could be even larger than $\pm 15 \%$ if the gluino mass is significantly lighter than the squark masses. Recent studies of the $B \rightarrow X_{d} \gamma$ rate asymmetry in specific models led to asymmetries between $-40 \%$ and $+40 \%$ (Akeroyd et al., 2001) and $-45 \%$ and $+21 \%$ (Asatryan and Asatrian, 1999; Asatryan et al., 2001). In general, $C P$ asymmetries may lead to clean evidence for new physics by a significant deviation from the standard-model prediction.

From Eq. (10.3), it is obvious that a large $C P$ asymmetry in the $B \rightarrow X_{s} \gamma$ channel or a positive $C P$ asymmetry in the inclusive $B \rightarrow X_{d} \gamma$ channel would be a clear signal for new physics.

The exclusive and inclusive decays of the form $b$ $\rightarrow s \gamma$ and $b \rightarrow d \gamma$, as well as their leptonic counterparts, provide a stringent test of whether the CKM matrix is indeed the only source of $C P$ violation. Using $U$ spin, which is the $\mathrm{SU}(2)$ subgroup of flavor $\mathrm{SU}(3)$ relating the $s$ and the $d$ quarks and which is a well-known tool in the context of nonleptonic decays (Fleischer, 1999; Gronau, 2000; Gronau and Rosner, 2001), one derives simple relations between the $C P$ asymmetries of the exclusive channels $B^{-} \rightarrow K^{*-} \gamma$ and $B^{-} \rightarrow \rho^{-} \gamma$ (Gronau, 2000) and of the inclusive channels $B \rightarrow X_{s} \gamma$ and $B \rightarrow X_{d} \gamma$ (Soares, 1991).

Any $C P$ violation in the standard model has to be proportional to

$$
\begin{aligned}
C= & i J\left(m_{u}-m_{c}\right)\left(m_{u}-m_{t}\right)\left(m_{c}-m_{t}\right)\left(m_{d}-m_{s}\right) \\
& \times\left(m_{d}-m_{b}\right)\left(m_{s}-m_{b}\right),
\end{aligned}
$$

where $J=\operatorname{Im}\left[V_{u b} V_{c b}^{*} V_{c s} V_{u s}^{*}\right]$ is the Jarlskog parameter. Therefore one should make use of the $U$-spin symmetry only with respect to the strong interactions. Defining the rate asymmetries (not the normalized $C P$ asymmetries) by

$$
\Delta \Gamma\left(B^{-} \rightarrow V^{-} \gamma\right)=\Gamma\left(B^{-} \rightarrow V^{-} \gamma\right)-\Gamma\left(B^{+} \rightarrow V^{+} \gamma\right),
$$


one arrives at the relation (Hurth and Mannel, 2001a)

$$
\Delta \Gamma\left(B^{-} \rightarrow K^{*-} \gamma\right)+\Delta \Gamma\left(B^{-} \rightarrow \rho^{-} \gamma\right)=b_{e x c} \Delta_{e x c},
$$

where the right-hand side is written as a product of a relative $U$-spin breaking $b_{e x c}$ and a typical size $\Delta_{e x c}$ of the $C P$-violating rate difference. This is a direct consequence of the unitarity of the CKM matrix and thus of the fact that the Jarlskog parameter is the only fourthorder quantity that is invariant under rephasing of the quark fields within the standard model. The resulting relation between $b \rightarrow s$ and $b \rightarrow d$ rate asymmetries due to

$$
J=\operatorname{Im}\left[\lambda_{u}^{(s)} \lambda_{c}^{(s) *}\right]=-\operatorname{Im}\left[\lambda_{u}^{(d)} \lambda_{c}^{(d) *}\right]
$$

was first noticed by Soares (1991).

Hurth and Mannel (2001a) derive the standard-model prediction for the difference of branching ratios, based on the model results of Greub et al. (1995) and on a sum-rule calculation of the form factors (Ali et al., 1994; Ball and Braun, 1998):

$$
\left|\Delta \mathcal{B}\left(B^{-} \rightarrow K^{*-} \gamma\right)+\Delta \mathcal{B}\left(B^{-} \rightarrow \rho^{-} \gamma\right)\right| \sim 4 \times 10^{-8}
$$

Note that the right-hand side is model dependent. The $U$-spin-breaking effects were also estimated in the QCD factorization approach (Bosch and Buchalla, 2002). Within this approach, it was shown that the $U$-spin-breaking effect essentially scales with the differences of the two form factors $\left(F_{K *}-F_{\rho}\right)$. Using the form factors from the QCD sum-rule calculation of Ball and Braun (1998) and maximizing the $C P$ asymmetries by a specific choice of the CKM angle $\gamma$, Bosch and Buchalla (2002) obtain

$$
\Delta \mathcal{B}\left(B^{-} \rightarrow K^{*-} \gamma\right)+\Delta \mathcal{B}\left(B^{-} \rightarrow \rho^{-} \gamma\right) \sim 3 \times 10^{-7},
$$

while for the separate asymmetries they obtain $\Delta \mathcal{B}(B$ $\left.\rightarrow K^{*} \gamma\right)=7 \times 10^{-7}$ and $\Delta \mathcal{B}(B \rightarrow \rho \gamma)=-4 \times 10^{-7}$, which explicitly shows the limitations of Eq. (9.7) as a test of the standard model.

The issue is much more attractive in the inclusive modes. Because of the heavy-mass expansion for the inclusive process, the leading contribution is the free $b$-quark decay. In particular, there is no sensitivity to the spectator quark and thus one arrives, within the partonic contribution, at the following relation for the $C P$ rate asymmetries as a consequence of CKM unitarity and of the real Wilson coefficients:

$$
\Delta \Gamma\left(B \rightarrow X_{s} \gamma\right)+\Delta \Gamma\left(B \rightarrow X_{d} \gamma\right)=b_{i n c} \Delta_{i n c} .
$$

In this framework one relies on parton-hadron duality. So one can actually compute the breaking of $U$ spin by keeping a nonvanishing strange-quark mass. The typical size of $b_{\text {inc }}$ can be roughly estimated to be of the order of $\left|b_{\text {inc }}\right| \sim m_{s}^{2} / m_{b}^{2} \sim 5 \times 10^{-4}$; here $\left|\Delta_{\text {inc }}\right|$ is again the average of the moduli of the two $C P$ rate asymmetries. These have been calculated (for vanishing strange-quark mass), e.g., by Ali et al. (1998), and one arrives at the following estimate within the partonic contribution (Hurth and Mannel, 2001a):

$$
\left|\Delta \mathcal{B}\left(B \rightarrow X_{s} \gamma\right)+\Delta \mathcal{B}\left(B \rightarrow X_{d} \gamma\right)\right| \sim 1 \times 10^{-9} .
$$

Going beyond the leading partonic contribution one has to check whether the large suppression factor from the $U$-spin breaking is still effective in addition to the natural suppression factors already present in the corresponding branching ratios. This question was addressed by Hurth and Mannel (2001b). In the leading $1 / m_{b}^{2}$ corrections, the $U$-spin-breaking effects also induce an additional overall factor $m_{s}^{2} / m_{b}^{2}$. In the nonperturbative corrections from the charm-quark loop, which scale with $1 / m_{c}^{2}$, one finds again the same overall suppression factor, because the operator $\widetilde{\mathcal{O}}$ [see Eq. (6.8)] does not contain any information on the strange mass. The corresponding long-distance contributions from up-quark loops, which scale with $\Lambda_{Q C D} / m_{b}$ (see Sec. VI), follow the same pattern. Thus, in the inclusive mode, the righthand side of Eq. (9.12) can be computed in a modelindependent way with the help of the heavy-mass expansion.

Therefore, the prediction (9.12) provides a very clean standard-model test, whether generic new $C P$ phases are active. Any significant deviation from the estimate (9.12) would be a direct hint of non-CKM contributions to $C P$ violation.

From the theoretical point of view, the sum of the $C P$ asymmetries in the inclusive $b \rightarrow s$ and $b \rightarrow d$ transitions turns out to be the favorable observable. This might also be true from the experimental point of view.

\section{FURTHER OPPORTUNITIES}

Generally, exclusive decay modes have large uncertainties due to the hadronic form factors, and it might thus be rather difficult to disentangle possible newphysics contributions from hadronic uncertainties in these modes - at least in the absence of very large new effects. Therefore exclusive modes can often be used only as QCD tests. However, there are exceptions to this rule. In specific ratios such as $C P$ asymmetries, hadronic uncertainties are reduced and large new-physics effects might be detectable. There are also exclusive modes that are as clean as inclusive modes because the corresponding hadronic matrix elements can be determined from experiment. The most important examples among these are the exclusive $B$ decay $B_{s} \rightarrow \mu^{+} \mu^{-}$and the exclusive rare kaon decays $K_{L} \rightarrow \pi^{0} \nu \bar{\nu}$ and $K^{+} \rightarrow \pi^{+} \nu \bar{\nu}$. The hadronic matrix elements of these FCNC (rare) processes can be related to well-known nonrare semileptonic decays.

Like the inclusive decay $B \rightarrow X_{s} \nu \bar{\nu}$ (see Sec. V), the exclusive decay $B_{s} \rightarrow \mu^{+} \mu^{-}$is dominated by the topquark contribution due to the hard GIM mechanism. QCD corrections within this exclusive mode are already calculated to NLL order. The remaining perturbative uncertainty is not larger than $\pm 1 \%$ (Buchalla and $\mathrm{Bu}-$ ras, 1993; Misiak and Urban, 1999). The corresponding hadronic matrix element is proportional to the decay constant of the $B_{s}$ meson, $f_{B}$, which can be determined on the lattice. The related uncertainty represents the largest part of the theoretical error: $f_{B_{s}}=(238$ 

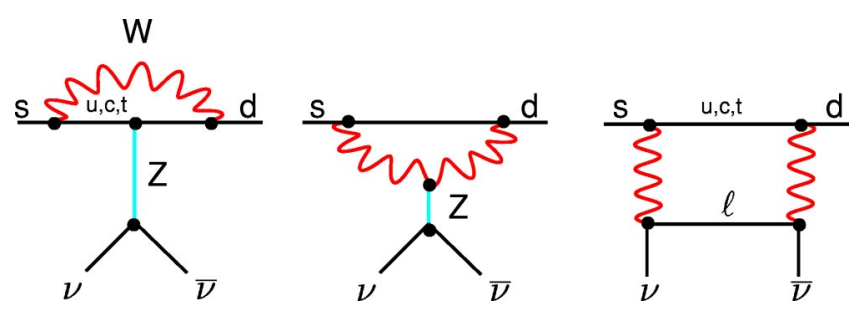

FIG. 35. (Color in online edition) One-loop diagrams contributing to $K \rightarrow \pi \nu \bar{\nu}$.

土31) MeV (Bernhard, 2001; Lellouch, 2001, 2002; Lellouch and Lüscher, 2001; Sachrajda, 2001). The standard-model prediction for the branching ratio of the decay $B_{s} \rightarrow \mu^{+} \mu^{-}$is of order $10^{-9}$. Thus this decay will be accessible at LHC and also at $B \mathrm{TeV}$. However, the branching ratio can be much larger within specific extensions of the standard model. For example, the helicity suppression of the standard-model contribution leads to an enhanced sensitivity to the Higgs-mediated scalar FCNC's within the two-Higgs-doublet model and especially within the MSSM. These nonstandard contributions lead to a drastic enhancement in the large $\tan \beta$ limit (Hamazaoui et al., 1999; Huang et al., 1999; Babu and Kolda, 2000; Isidori and Retico, 2001; Buras, Chankowski, et al., 2002; Dedes and Pilaftsis, 2003). Therefore, this decay might even be detectable at Fermilab before the LHC experiments and the $B \mathrm{TeV}$ experiments start to take data (see Choudhury and Gaur, 1999; Bobeth etal., 2001; Chankowski and Slawianowska, 2001; Dedes et al., 2001; Huang et al., 2001; Xiong and Yang, 2002 for further discussions).

The other two important examples of theoretically clean exclusive modes, $K_{L} \rightarrow \pi^{0} \nu \bar{\nu}$ and $K^{+} \rightarrow \pi^{+} \nu \bar{\nu}$, are discussed in more detail in the following.

The rare decays $K_{L} \rightarrow \pi^{0} \nu \bar{\nu}$ and $K^{+} \rightarrow \pi^{+} \nu \bar{\nu}$ represent complementary opportunities for precision flavor physics. They are also FCNC processes induced at the oneloop level via $Z^{0}$ penguin and box diagrams (see Fig. 35) and are exceptionally clean processes.

As in the inclusive decay $B \rightarrow X_{s} \nu \bar{\nu}$ (see discussion in Sec. V, including footnote), the hard GIM mechanism is active: the short-distance top and charm contributions dominate the long-distance up-quark contribution within the charged mode $K^{+} \rightarrow \pi^{+} \nu \bar{\nu}$. The CKM factors of the charm contribution compensate for the hard GIM suppression relative to the top contribution in this specific case. The short-distance amplitude is then governed by one single semileptonic operator, namely, $\left(\bar{s} \gamma_{\mu} P_{L} d\right)\left(\bar{\nu} \gamma_{\mu} P_{L} \nu\right)$. Its hadronic matrix element can be determined experimentally by the semileptonic kaon decay. In fact, the matrix element $\left\langle\pi^{+}\left|\bar{s} \gamma_{\mu} P_{L} d\right| K^{+}\right\rangle$can be related by isospin symmetry to the matrix element $\sqrt{2}\left\langle\pi^{0}\left|\bar{s} \gamma_{\mu} P_{L} u\right| K^{+}\right\rangle$of the semileptonic decay $K^{+}$ $\rightarrow \pi^{0} e^{+} \nu$. The corresponding Wilson coefficient is already calculated to next-to-leading logarithm QCD (Buchalla and Buras, 1993; Misiak and Urban, 1999) and the scale dependence is reduced to $5 \%$ in the chargedkaon mode.
The situation is even more favorable in the neutral mode, which is dominated by the $C P$-violating part. There is no relative CKM enhancement of the charm contribution, and thus the amplitude is dominated by the top contribution as in the inclusive rare $B \rightarrow X_{s} \bar{\nu} \nu$ decay. The NLL QCD calculation therefore leads to only a $1 \%$ scale uncertainty (Buchalla and Buras, 1993; Misiak and Urban, 1999).

The validity of the operator-product expansion and the renormalization-group-improved perturbation theory in the charm contribution to the charged mode has been critically analyzed: the separate scale dependence within the charm contribution of $13 \%$ at NLL QCD is consistent with partial NNLL results (Buchalla, 2001). Moreover, subleading power corrections within the operator-product expansion of order $m_{K}^{2} / m_{c}^{2}$-which might lead to $15 \%$ correction-are estimated to be at the level of $5 \%$. However, for a reliable determination of the latter corrections, a lattice calculation of the corresponding hadronic matrix elements will be indispensable (Falk et al., 2001).

The latest numerical standard-model predictions are (Buchalla and Buras, 1999; D'Ambrosio and Isidori, 2002)

$$
\begin{aligned}
& \mathcal{B}\left(K^{+} \rightarrow \pi^{+} \nu \bar{\nu}\right)=(7.2 \pm 2.1) \times 10^{-11}, \\
& \mathcal{B}\left(K_{L} \rightarrow \pi^{0} \nu \bar{\nu}\right)=(2.8 \pm 1.1) \times 10^{-11} .
\end{aligned}
$$

The uncertainties of the present standard-model predictions are dominated by the current errors of the CKM parameters, while the instrinsic error in the charged mode is about $6 \%$ (mainly from the charm contribution) and in the neutral mode only about $2 \%$. This implies the importance of these decay modes for CKM phenomenology: they play a unique role among $K$ decays, as does the $B_{d} \rightarrow \psi K_{S}$ mode among $B$ decays. The measurements of the two kaon decay modes allow for a measurement of the angle $\beta$ of the unitarity triangle to a precision comparable to that obtained with the $B_{d}$ $\rightarrow \psi K_{S}$ mode before the LHC era (Buchalla and Buras, 1994). The only necessary theoretical input is the internal charm contribution to $K^{+} \rightarrow \pi^{+} \nu \bar{\nu}$, which introduces some theoretical uncertainty (see above).

The relation $(\sin 2 \beta)_{\pi \nu \bar{\nu}}=(\sin 2 \beta)_{\psi K_{S}}$ implies a very interesting connection between rare $K$ decays and $B$ physics, which must be satisfied in the standard model:

$$
(\sin 2 \beta)_{\pi \nu \bar{\nu}}=(\sin 2 \beta)_{\psi K_{s}}=-A_{C P}\left(\psi K_{S}\right) \frac{1+x_{d}^{2}}{x_{d}} .
$$

Here $A_{C P}\left(\psi K_{S}\right)$ denotes the time-integrated $C P$-violating asymmetry in $B_{d}^{0} \rightarrow \psi K_{S}$ and $x_{d}=\Delta m / \Gamma$ gives the size of $B_{d}^{0}-\bar{B}_{d}^{0}$ mixing. As was stressed by Buchalla and Buras (1994), besides the internal charm contribution to the charged-kaon mode, all quantities in Eq. (10.2) can be directly measured experimentally, and their relation is almost independent of $V_{c b}$.

Besides their rich CKM phenomenology, the decays $K_{L} \rightarrow \pi^{0} \nu \bar{\nu}$ and $K^{+} \rightarrow \pi^{+} \nu \bar{\nu}$ as loop-induced processes are very sensitive to new physics beyond the standard 


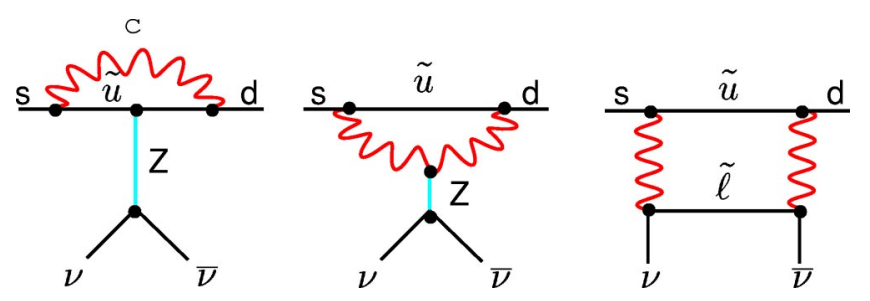

FIG. 36. (Color in online edition) Supersymmetric contributions to $K \rightarrow \pi \nu \bar{\nu}$.

model. In addition, the theoretical information is very clean and the measurement of these decays thus leads to very accurate constraints on any new-physics model. Moreover, there is the possibility that these clean rare decay modes could themselves lead to first evidence of new physics when the measured decay rates are not compatible with the standard model.

New-physics contributions in $K_{L} \rightarrow \pi^{0} \nu \bar{\nu}$ and $K^{+}$ $\rightarrow \pi^{+} \nu \bar{\nu}$ can be parametrized in a model-independent way by two parameters that quantify the violation of Eq. (10.2) (Buras, Romanino, and Silvestrini, 1998; Nir and Worah, 1998). New effects in supersymmetric models can be induced through new box- and penguin-diagram contributions which involve new particles such as charged Higgs or charginos and stops (Fig. 36) that replace the $W$ boson and the up-type quark of the standard model (Fig. 35).

Under the simplifying MFV assumption of D'Ambrosio and Isidori (2002; see Sec. VIII.A), Eq. (10.2) is valid. Thus the measurements of $\mathcal{B}\left(K_{L}\right.$ $\left.\rightarrow \pi^{0} \nu \bar{\nu}\right)$ and $\mathcal{B}\left(K^{+} \rightarrow \pi^{+} \nu \bar{\nu}\right)$ still directly determine the angle $\beta$, and a significant violation of Eq. (10.2) would rule out this assumption.

For the present experimental status of supersymmetry, however, a model-independent analysis that also includes a general flavor change through the squark mass matrices is more suitable. If the new sources of flavor change are parametrized by the mass-insertion approximation, an expansion of the squark mass matrices around their diagonal, it turns out that supersymmetric contributions in this more general setting of the unconstrained MSSM allow for a significant violation of Eq. (10.2). An enhancement of the branching ratios by an order of magnitude (in the case of $K^{+} \rightarrow \pi^{+} \nu \bar{\nu}$ by a factor of 3) with respect to the standard-model values is possible, mostly thanks to the chargino-induced $Z$-penguin contribution (Colangelo and Isidori, 1998). Recent analyses (Colangelo and Isidori, 1998; Buras and Silvestrini, 1999; Buras et al., 2000) within the unconstrained MSSM focused on the correlation of rare decays and $\epsilon^{\prime} / \epsilon$ and led to reasonable upper bounds for the branching ratios $\mathcal{B}\left(K_{L} \rightarrow \pi^{0} \nu \bar{\nu}\right) \leqslant 1.2 \times 10^{-10}$ and $\mathcal{B}\left(K^{+} \rightarrow \pi^{+} \nu \bar{\nu}\right) \leqslant 1.7 \times 10^{-10}$, which should be compared with the latest numerical standard-model predictions [Eq. (10.1)].

The rare decays $K^{+} \rightarrow \pi^{+} \nu \bar{\nu}$ and $K_{L} \rightarrow \pi^{0} \nu \bar{\nu}$ are specifically interesting in view of the suggested experiments at the Brookhaven National Laboratory (Kudenko, 2001; E949 Collaboration, 2003), at Fermilab (Cooper,
2001), and at KEK (Abe et al., 2000). [See Barker and Kettell (2000) for a review.]

For the neutral $K_{L} \rightarrow \pi^{0} \nu \bar{\nu}$ mode, the experimental situation is not yet satisfactory; there is only an upper bound available from $K \mathrm{TeV}$ (Alavi-Harati et al., 2000):

$$
\mathcal{B}\left(K_{L} \rightarrow \pi^{0} \nu \bar{\nu}\right)<5.9 \times 10^{-7},
$$

which is four orders of magnitude above the standardmodel expectation. An indirect upper bound on $\mathcal{B}\left(K_{L}\right.$ $\left.\rightarrow \pi^{0} \nu \bar{\nu}\right)$, using the current limit on $\mathcal{B}\left(K^{+} \rightarrow \pi^{+} \nu \bar{\nu}\right)$ and isospin symmetry, can be placed at $2.6 \times 10^{-9}$ (Grossman and Nir, 1997). Future prospects are given by the E391a experiment at KEK with a sensitivity of $3 \times 10^{-10}$ (possible start in 2003; Abe et al., 2000) and the E926 experiment (KOPIO) at Brookhaven, which aims at a sensitivity of $10^{-13}$ (Kudenko, 2001).

For the charged $K^{+} \rightarrow \pi^{+} \nu \bar{\nu}$ mode, the experimental situation is more favorable. The current Brookhaven experiment E787 has, to date, observed two clean candidate events for $K^{+} \rightarrow \pi^{+} \nu \bar{\nu}$. The combined analysis including previous data (Adler et al., 2000) leads to the branching ratio (Adler et al., 2002)

$$
\mathcal{B}\left(K^{+} \rightarrow \pi^{+} \nu \bar{\nu}\right)=\left(1.57_{-0.82}^{+1.75}\right) \times 10^{-10} .
$$

The central value is more than twice the central value of the theoretical standard-model prediction, but the present measurement is still compatible with it, in view of the large error bars on the experimental side. Figure 37 illustrates the possible future impact of more precise measurements-to be expected from the Brookhaven experiment E949 with a sensitivity of $10^{-11}$ /event (started in 2001) $(E 949,2003)$ and from the future highprecision CKM experiment at Fermilab with yet an order-of-magnitude higher sensitivity (starting in 2007; Cooper, 2001). If the present central value is confirmed with a smaller error, this would clearly indicate a newphysics contribution either in $B \bar{B}$ mixing or in the $K^{+}$ $\rightarrow \pi^{+} \nu \bar{\nu}$ mode (D'Ambrosio and Isidori, 2002).

\section{SUMMARY}

In this paper we have reviewed the status of inclusive rare $B$ decays, highlighting recent developments. These decays give special insight into the CKM matrix; moreover, as FCNC processes, they are loop induced and therefore particularly sensitive to new physics.

Decay modes such as $B \rightarrow X_{s} \gamma, B \rightarrow X_{s} \nu \bar{\nu}$, and $B$ $\rightarrow X_{s} \ell^{+} \ell^{-}$(with specific kinematic cuts) are dominated by the partonic (perturbative) contributions and are thus theoretically very clean, in contrast to the corresponding exclusive decay modes, and serve as laboratories to search for new physics. Nonperturbative contributions play a subordinate role and they are under control, thanks to the heavy-mass expansion. The inclusive rare $B$ decays are or will be accessible at the present $e^{+} e^{-}$ machines (CLEO, BABAR, BELLE), with their low background and their kinematic constraints, and will make precision flavor physics possible in the near future.

Significant theoretical progress has been made during recent years. Calculations of NLL (or even NNLL) 


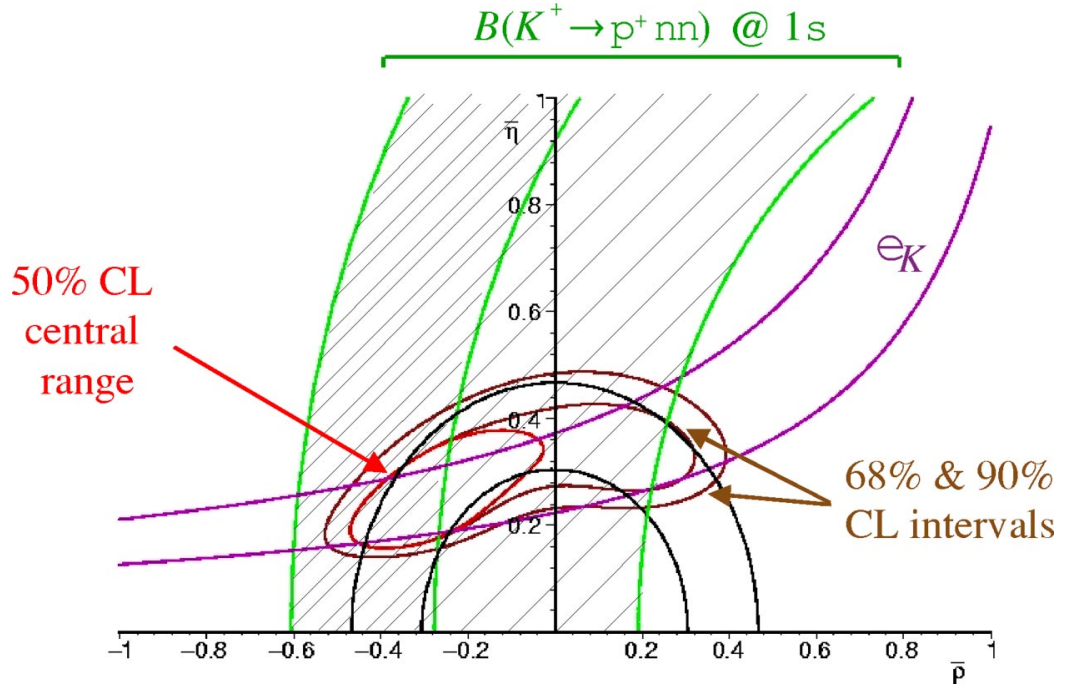

FIG. 37. (Color in online edition) Allowed region in the $\bar{\rho}-\bar{\eta}$ plane with the inclusion of the latest $K^{+} \rightarrow \pi^{+} \nu \bar{\nu}$ and without $B_{s, d}$ data. The two external contours denote $68 \%$ and $90 \%$ confidence intervals; the inner one is the $68 \%$ confidence interval under the assumption that the experimental error in the present measurement is reduced by a factor of 2 . From D'Ambrosio and Isidori, 2002.
QCD corrections to these decay modes have been performed. The theoretical uncertainty has been significantly reduced. As was emphasized, the step from LL to NLL precision within the framework of the renormalization-group-improved perturbation theory is not only a quantitative, but also a qualitative one, which tests the validity of the perturbative approach in a given problem.

Within the theoretical prediction of $B \rightarrow X_{s} \gamma$, the charm-mass renormalization-scheme ambiguity at NLL order represents the largest uncertainty. In view of the precise experimental data coming up from the $B$ factories in the near future, this uncertainty should be removed.

Inclusive rare $B$ decays allow for an indirect search for new physics, a strategy complementary to the direct production of new (supersymmetric) particles, which is reserved for the planned hadronic machines such as the LHC at CERN. But the indirect search at the $B$ factories already implies significant restrictions for the parameter space of supersymmetric models and thus leads to important theoretically clean information for the direct search for supersymmetric particles.

It is even possible that these rare processes might give the first evidence of new physics outside the neutrino sector by a significant deviation from the standardmodel prediction. But in the long run, after new physics has already been discovered, inclusive rare $B$ decays will also play an important role in analyzing in greater detail the underlying new dynamics.

Within supersymmetric models, the QCD calculation of the inclusive rare $B$ decays has not reached the sophistication of the corresponding standard-model calculations. Nevertheless, NLL analyses in specific scenarios already show that bounds on the parameter space of nonstandard models are rather sensitive to NLL QCD contributions.

Detailed measurements of $C P$ asymmetries in rare $B$ decays will also be possible in the near future. They will allow for a stringent and clean test of whether the CKM matrix is indeed the only source of $C P$ violation. Moreover, a measurement of the photon polarization within the rare $B$ decays will be possible in order to check the standard-model prediction of a left-handed photon.

The rare-kaon decays, $K^{+} \rightarrow \pi^{+} \nu \bar{\nu}$ and $K_{L} \rightarrow \pi^{0} \nu \bar{\nu}$, offer complementary opportunities for precision flavor physics. Besides the current Brookhaven experiment, several more are planned or suggested to explore these theoretically clean decay modes.

\section{ACKNOWLEDGMENTS}

I thank Colin Jessop for many helpful discussions on the experimental aspects of inclusive rare $B$ decays and Ed Thorndike and Kenichi Nakao for useful comments. I am very grateful to Mikolaj Misiak for his careful reading of the manuscript and for useful comments. Discussions with Thomas Besmer, Francesca Borzumati, Giancarlo D'Ambrosio, Paolo Gambino, Adrian Ghinculov, Christoph Greub, Gino Isidori, Thomas Mannel, Daniel Wyler, and York-Peng Yao are also gratefully acknowledged.

\section{REFERENCES}

Abe, K., et al., (KEK-PS E391a Collaboration), 2000, "Status of the $K_{L}^{0} \rightarrow \pi^{0} \nu \bar{\nu}$ experiment at KEK (E391a)," KEKPREPRINT-2000.

Abe, K., et al., (BELLE Collaboration), 2001a, "A measurement of the branching fraction for the inclusive $B \rightarrow X_{s} \gamma$ decays with BELLE," Phys. Lett. B 511, 151.

Abe, K., et al., (BELLE Collaboration), 2001b, "Evidence for the electroweak penguin decay $B \rightarrow X_{s} \ell^{+} \ell^{-}$," hep-ex/0107072.

Abe, K., et al., (BELLE Collaboration), 2002a, "Observation of the decay $B \rightarrow K \ell^{+} \ell^{-}$," Phys. Rev. Lett. 88, 021801.

Abe, K., et al., (BELLE Collaboration), 2002b, “An improved measurement of mixing-induced $C P$ violation in the neutral $B$-meson system," Phys. Rev. D 66, 071102.

Abe, K., et al., (BELLE Collaboration), 2002c, "Measurement of the $B \rightarrow K^{*} \gamma$ branching fractions," BELLE-CONF-0239. 
Abe, K., et al., (BELLE Collaboration), 2002d, "Study of radiative $B$ decays into nonstrange final states," BELLECONF-0240.

Abe, K., et al., (BELLE Collaboration), 2002e, "Study of electroweak penguin decay measurement of the $B \rightarrow K^{*} \ell^{+} \ell^{-}$," BELLE-CONF-0241.

Abud, M., G. Ricciardi, and G. Sterman, 1998, "Light masses in short-distance penguin loops," Phys. Lett. B 437, 169.

Adel, K., and Y. Yao, 1994, "Exact $\alpha_{s}$ calculation of $b \rightarrow s \gamma$, $b \rightarrow s$ gluon," Phys. Rev. D 49, 4945.

Adler, S., et al., (E787 Collaboration), 2000, "Further search for the decay $K^{+} \rightarrow \pi^{+} \nu \bar{\nu}$," Phys. Rev. Lett. 84, 3768; see also http://www.phy.bnl.gov/e787/e787.html

Adler, S., et al., (E787 Collaboration), 2002, "Further evidence for the decay $K^{+} \rightarrow \pi^{+} \nu \bar{\nu}$," Phys. Rev. Lett. 88, 041803.

Aglietti, U., M. Ciuchini, and P. Gambino, 2002, "A new model-independent way of extracting $|V(u b) / V(c b)|$," Nucl. Phys. B 637, 427.

Aglietti, U., and G. Ricciardi, 2000, "The structure function of semi-inclusive heavy flavour decays in field theory," Nucl. Phys. B 587, 363.

Ahmed, S., et al., (CLEO Collaboration), 1999, " $b \rightarrow s \gamma$ branching fraction and CP asymmetry," hep-ex/9908022.

Akeroyd, A. G., Y. Y. Keum, and S. Recksiegel, 2001, "Effect of supersymmetric phases on the direct $C P$ asymmetry of $B$ $\rightarrow X_{d} \gamma$," Phys. Lett. B 507, 252.

Alam, M. S., et al., (CLEO Collaboration), 1995, "First measurement of the rate for the inclusive radiative penguin decay $b \rightarrow s \gamma$," Phys. Rev. Lett. 74, 2885.

Alavi-Harati, A., et al., (E799-II/KTeV Collaboration), 2000, "Search for the decay $K_{L} \rightarrow \pi^{0} \nu \bar{\nu}$ using $\pi^{0} \rightarrow e^{+} e^{-} \gamma$," Phys. Rev. D 61, 072006; see also http://kpasa.fnal.gov:8080/public/ ktev.html

ALEPH Collaboration, 1996, Report No. PA10-019, presented at the 28th International Conference on High Energy Physics, 25-31 July, 1996, Warsaw, Poland.

Ali, A., 1997, "Theory of rare B decays," hep-ph/9709507.

Ali, A., H. Asatrian, and C. Greub, 1998, "Inclusive decay rate for $B \rightarrow X_{d} \gamma$ in next-to-leading logarithmic order and $C P$ asymmetry in the standard model," Phys. Lett. B 429, 87.

Ali, A., P. Ball, L. T. Handoko, and G. Hiller, 2000, "A comparative study of the decays $B \rightarrow\left(K, K^{*}\right) \ell^{+} \ell^{-}$in standard model and supersymmetric theories," Phys. Rev. D 61, 074024.

Ali, A., and V. M. Braun, 1995, "Estimates of the weak annihilation contributions to the decays $B \rightarrow \rho \gamma$ and $B \rightarrow \omega \gamma$," Phys. Lett. B 359, 223.

Ali, A., V. M. Braun, and H. Simma, 1994, "Exclusive radiative $B$ decays in the light cone QCD sum rule approach," Z. Phys. C 63, 437.

Ali, A., G. F. Giudice, and T. Mannel, 1995, "Towards a modelindependent analysis of rare B decays," Z. Phys. C 67, 417.

Ali, A., and C. Greub, 1991 "Inclusive photon energy spectrum in rare $B$ decays," Z. Phys. C 49, 431.

Ali, A., and C. Greub, 1995, "Photon energy spectrum in $B$ $\rightarrow X_{s} \gamma$ and comparison with data," Phys. Lett. B 361, 146.

Ali, A., and G. Hiller, 1999, "A theoretical reappraisal of branching ratios and $C P$ asymmetries in the decays $B$ $\rightarrow\left(X_{d}, X_{s}\right) \ell^{+} \ell^{-}$and determination of the CKM parameters," Eur. Phys. J. C 8, 619.
Ali, A., G. Hiller, L. T. Handoko, and T. Morozumi, 1997, "Power corrections in the decay rate and distributions in $B$ $\rightarrow X_{s} \ell^{+} \ell^{-}$in the standard model," Phys. Rev. D 55, 4105 .

Ali, A., E. Lunghi, C. Greub, and G. Hiller, 2002, "Improved model-independent analysis of semileptonic and radiative rare $B$ decays," Phys. Rev. D 66, 034002.

Ali, A., and A. Y. Parkhomenko, 2002, "Branching ratios for $B \rightarrow \rho \gamma$ decays in next-to-leading order in $\alpha_{s}$ including hard spectator corrections," Eur. Phys. J. C 23, 89.

Ali, A., and E. Pietarinen, 1979, "Semileptonic decays of heavy quarks in quantum chromodynamics," Nucl. Phys. B 154, 519. Altarelli, G., N. Cabibbo, G. Corbo, L. Maiani, and G. Martinelli, 1982, "Leptonic decay of heavy flavors: A theoretical update," Nucl. Phys. B 208, 365.

Ammar, R., et al., (CLEO Collaboration), 1993, "Evidence for penguins: First observation of $B \rightarrow K^{*}(892) \gamma$," Phys. Rev. Lett. 71, 674.

Anikeev, K., et al., 2002, " $B$ physics at the Tevatron: Run II and beyond," hep-ph/0201071.

Aoki, M., G. Cho, and N. Oshimo, 1999, “CP asymmetry for radiative $B$-meson decay in the supersymmetric standard model," Nucl. Phys. B 554, 50.

Asatrian, H. M., K. Bieri, C. Greub, and A. Hovhannisyan, 2002, "NNLL corrections to the angular distribution and to the forward-backward asymmetries in $b \rightarrow X_{s} \ell^{+} \ell^{-}$," Phys. Rev. D 66, 094013.

Asatryan, H. H., and H. M. Asatrian, 1999, " $C P$ asymmetry for inclusive decay $B \rightarrow X_{d} \gamma$ in the minimal supersymmetric standard model," Phys. Lett. B 460, 148.

Asatryan, H. H., H. M. Asatrian, C. Greub, and M. Walker, 2001, "Two-loop virtual corrections to $B \rightarrow X_{s} \ell^{+} \ell^{-}$in the standard model," Phys. Lett. B 507, 162.

Asatryan, H. H., H. M. Asatrian, C. Greub, and M. Walker, 2002, "Complete gluon bremsstrahlung corrections to the process $b \rightarrow s \ell^{+} \ell^{-}$," Phys. Rev. D 66, 034009.

Asatryan, H. H., H. M. Asatrian, G. K. Yeghiyan, and G. K. Savvidy, 2001, "Direct $C P$ asymmetry in inclusive rare $B$ decays in 2HDM," Int. J. Mod. Phys. A 16, 3805.

Atwood, D., M. Gronau, and A. Soni, 1997, "Mixing-induced $C P$ asymmetries in radiative $B$ decays in and beyond the standard model," Phys. Rev. Lett. 79, 185.

Aubert, B., et al., (BABAR Collaboration), 2002a, "Measurement of $B \rightarrow K^{*} \gamma$ branching fractions and charge asymmetries," Phys. Rev. Lett. 88, 101805.

Aubert, B., et al., (BABAR Collaboration), 2002b, "Measurement of the $C P$-violating asymmetry amplitude $\sin 2 \beta$," Phys. Rev. Lett. 89, 201802.

Aubert, B., et al., (BABAR Collaboration), 2002c, "Search for the exclusive radiative decays $B \rightarrow \rho \gamma$ and $B^{0} \rightarrow \omega \gamma$," hep-ex/0207073.

Aubert, B., et al., (BABAR Collaboration), 2002d, "Determination of the branching fraction for inclusive decays $B$ $\rightarrow X_{s} \gamma$," hep-ex/0207076.

Aubert, B., et al., (BABAR Collaboration), 2002e, "Evidence for the flavor changing neutral current decays $B \rightarrow K \ell^{+} \ell^{-}$ and $B \rightarrow K^{*} \ell^{+} \ell^{-}$," hep-ex/0207082.

Aubert, B., et al., (BABAR Collaboration), 2002f, " $b \rightarrow s \gamma$ using a sum of exclusive modes," hep-ex/0207074.

BABAR Collaboration, 2003, http://www.slac.stanford.edu/ BFROOT/ 
Babu, K. S., and C. F. Kolda, 2000, "Higgs-mediated $B^{0}$ $\rightarrow \mu^{+} \mu^{-}$in minimal supersymmetry," Phys. Rev. Lett. 84, 228.

Baek, S., and P. Ko, 1999, "Probing SUSY-induced $C P$ violations at $B$ factories," Phys. Rev. Lett. 83, 488.

Baer, H., M. Brhlik, D. Castano, and X. Tata, 1998, " $b \rightarrow s \gamma$ constraints on the minimal supergravity model with large $\tan (\beta)$," Phys. Rev. D 58, 015007.

Bailin, D., and S. Khalil, 2001, "Flavor-dependent SUSY phases and $C P$ asymmetry in $B \rightarrow X_{s} \gamma$ decays," Phys. Rev. Lett. 86, 4227.

Ball, P., and V. M. Braun, 1998, "Exclusive semileptonic and rare B-meson decays in QCD," Phys. Rev. D 58, 094016.

Ball, P., et al., 2000, "B decays at the LHC," hep-ph/0003238.

Baranowski, K., and M. Misiak, 2000, "The $O\left(\alpha_{e m} / \alpha_{s}\right)$ correction to $\mathcal{B}\left(B \rightarrow X_{s} \gamma\right)$," Phys. Lett. B 483, 410 .

Barate, R., et al., (ALEPH Collaboration), 1998, "A measurement of the inclusive $b \rightarrow s \gamma$ branching ratio," Phys. Lett. B 429, 169.

Barbieri, R., G. Dvali, and L. J. Hall, 1996, "Predictions from a $\mathrm{U}(2)$ flavour symmetry in supersymmetric theories," Phys. Lett. B 377, 76.

Barbieri, R., S. Ferrara, and C. A. Savoy, 1982, "Gauge models with spontaneously broken local supersymmetry," Phys. Lett. B 119, 343.

Barker, A. R., and S. H. Kettell, 2000, "Developments in rare kaon decay physics," Annu. Rev. Nucl. Part. Sci. 50, 249.

Bartl, A., T. Gajdosik, E. Lunghi, A. Masiero, W. Porod, H. Stremnitzer, and O. Vives, 2001, "General flavor-blind MSSM and CP violation,” Phys. Rev. D 64, 076009.

Bauer, C. W., and C. N. Burrell, 2000, "Nonperturbative corrections to moments of the decay $B \rightarrow X_{s} \ell^{+} \ell^{-}$," Phys. Rev. D 62, 114028.

Bauer, C. W., M. Luke, and T. Mannel, 2002, "Subleading shape functions in $B \rightarrow X_{u} \ell \bar{\nu}$ and the determination of $|V(u b)|$," Phys. Lett. B 43, 261.

Bauer, C. W., D. Pirjol, and I. W. Stewart, 2001 “A proof of factorization for $B \rightarrow D \pi$," Phys. Rev. Lett. 87, 201806.

BELLE Collaboration, 2003, http://belle.kek.jp/

Belyaev, A., et al., (Kaon Physics Working Group Collaboration), 2001, "Kaon physics with a high-intensity proton driver," hep-ph/0107046.

Beneke, M., G. Buchalla, M. Neubert, and C. T. Sachrajda, 2000, "QCD factorization for exclusive, nonleptonic $B$-meson decays: General arguments and the case of heavy-light final states," Nucl. Phys. B 591, 313.

Beneke, M., G. Buchalla, M. Neubert, and C. T. Sachrajda, 2001, "QCD factorization in $B \rightarrow \pi K, \pi \pi$ decays and extraction of Wolfenstein parameters," Nucl. Phys. B 606, 245.

Beneke, M., A. P. Chapovsky, M. Diehl, and T. Feldmann, 2002, "Soft-collinear effective theory and heavy-to-light currents beyond leading power," Nucl. Phys. B 643, 431.

Beneke, M., T. Feldmann, and D. Seidel, 2001, "Systematic approach to exclusive $B \rightarrow V \ell^{+} \ell^{-}, V \gamma$ decays," Nucl. Phys. B 612, 25.

Bennett, G. W., et al., (Muon g-2 Collaboration), 2002, "Measurement of the positive muon anomalous magnetic moment to 0.7 ppm," Phys. Rev. Lett. 89, 101804; 89, 129903(E).

Bernard, C., 2001, "Heavy quark physics on the lattice," Nucl. Phys. B. (Proc. Suppl.) 94A, 159.
Bertolini, S., F. Borzumati, A. Masiero, and G. Ridolfi, 1991, "Effects of supergravity induced electroweak breaking on rare $B$ decays and mixings," Nucl. Phys. B 353, 591.

Besmer, T., C. Greub, and T. Hurth, 2001, "Bounds on flavour violating parameters in supersymmetry," Nucl. Phys. B 609, 359.

Bigi, I. I., M. A. Shifman, and N. Uraltsev, 1997, "Aspects of heavy quark theory,” Annu. Rev. Nucl. Part. Sci. 47, 591.

Bigi, I. I., M. Shifman, N. G. Uraltsev, and A. Vainshtein, 1993, "QCD predictions for lepton spectra in inclusive heavy flavor decays," Phys. Rev. Lett. 71, 496.

Bigi, I. I., M. Shifman, N. G. Uraltsev, and A. Vainshtein, 1994, "On the motion of heavy quarks inside hadrons: Universal distributions and inclusive decays," Int. J. Mod. Phys. A 9, 2467.

Bigi, I. I., and N. Uraltsev, 2001, "A vademecum on quark hadron duality,” Int. J. Mod. Phys. A 16, 5201.

Bigi, I. I., N. G. Uraltsev, and A. I. Vainshtein, 1992, "Nonperturbative corrections to inclusive beauty and charm decays: QCD versus phenomenological models," Phys. Lett. B 293, 430; 297, 477(E).

Bjorken, J. D., 1989, "Topics in B Physics," Nucl. Phys. B. (Proc. Suppl.) 11A, 325.

Bobeth, C., A. J. Buras, F. Kruger, and J. Urban, 2002, "QCD corrections to $\bar{B} \rightarrow X_{d, s} \nu \bar{\nu}, B_{d, s} \rightarrow \ell^{+} \ell^{-}, K \rightarrow \pi \nu \bar{\nu}$ and $K_{L}$ $\rightarrow \mu^{+} \mu^{-}$in the MSSM," Nucl. Phys. B 630, 87.

Bobeth, C., T. Ewerth, F. Kruger, and J. Urban, 2001, “Analysis of neutral Higgs-boson contributions to the decays $\bar{B}_{s}$ $\rightarrow \ell^{+} \ell^{-}$and $\bar{B} \rightarrow K \ell^{+} \ell^{-}$," Phys. Rev. D 64, 074014.

Bobeth, C., M. Misiak, and J. Urban, 2000a, "Matching conditions for $b \rightarrow s \gamma$ and $b \rightarrow s$ gluon in extensions of the standard model," Nucl. Phys. B 567, 153.

Bobeth, C., M. Misiak, and J. Urban, 2000b, "Photonic penguins at two loops and $m_{t}$ dependence of $\mathcal{B}\left(B \rightarrow X_{s} \ell^{+} \ell^{-}\right)$," Nucl. Phys. B 574, 291.

Bornheim, A., et al., (CLEO Collaboration), 2002, "Improved measurement of $|V(u b)|$ with inclusive semileptonic $B$ decays,” Phys. Rev. Lett. 88, 231803.

Borzumati, F. M., 1994, "The decay $b \rightarrow s \gamma$ in the MSSM revisited,” Z. Phys. C. 63, 291.

Borzumati, F. M., and C. Greub, 1998, "2HDMs predictions for $\bar{B} \rightarrow X_{s} \gamma$ in NLO QCD," Phys. Rev. D 58, 074004.

Borzumati, F. M., C. Greub, T. Hurth, and D. Wyler, 2000, "Gluino contribution to radiative $B$ decays: Organization of QCD corrections and leading-order results," Phys. Rev. B 62, 075005.

Bosch, S. W., and G. Buchalla, 2002, "The radiative decays $B$ $\rightarrow V \gamma$ at next-to-leading order in QCD," Nucl. Phys. B 621, 459.

Braun, V. M., 1998, "Light-cone sum rules," hep-ph/9801222. Buchalla, G., 2001, "Rare kaon decays: Overview," hep-ph/0110313.

Buchalla, G., and A. J. Buras, 1993, "QCD corrections to rare $K$ and $B$ decays for arbitrary top quark mass," Nucl. Phys. B 400, 225.

Buchalla, G., and A. J. Buras, 1994, " $\sin 2 \beta$ from $K \rightarrow \pi \nu \bar{\nu}$," Phys. Lett. B 333, 221.

Buchalla, G., and A. J. Buras, 1999, "The rare decays $K$ $\rightarrow \pi \nu \bar{\nu}, B \rightarrow X \nu \bar{\nu}$ and $B \rightarrow \ell^{+} \ell^{-}:$An update," Nucl. Phys. B 548, 309. 
Buchalla, G., and G. Isidori, 1998, "Nonperturbative effects in $\bar{B} \rightarrow X_{s} \ell^{+} \ell^{-}$for large dilepton invariant mass," Nucl. Phys. B 525, 333.

Buchalla, G., G. Isidori, and S. J. Rey, 1998, "Corrections of order $\Lambda_{Q C D}^{2} / m_{c}^{2}$ to inclusive rare $B$ decays," Nucl. Phys. B 511, 594.

Buras, A. J., 1998, "Weak Hamiltonian, CP violation and rare decays," hep-ph/9806471.

Buras, A. J., P. H. Chankowski, J. Rosiek, and L. Slawianowska, 2002, "Correlation between $\Delta M_{s}$ and $B_{s, d}^{0}$ $\rightarrow \mu^{+} \mu^{-}$in supersymmetry at large $\tan (\beta)$, " Phys. Lett. B 546, 96.

Buras, A. J., G. Colangelo, G. Isidori, A. Romanino, and L. Silvestrini, 2000, "Connections between $\epsilon^{\prime} / \epsilon$ and rare kaon decays in supersymmetry," Nucl. Phys. B 566, 3.

Buras, A. J., A. Czarnecki, M. Misiak, and J. Urban, 2001, "Two-loop matrix element of the current-current operator in the decay $b \rightarrow X_{s} \gamma$," Nucl. Phys. B 611, 488.

Buras, A. J., A. Czarnecki, M. Misiak, and J. Urban, 2002, "Completing the NLO QCD calculation of $\bar{B} \rightarrow X_{s} \gamma$," Nucl. Phys. B 631, 219.

Buras, A. J., A. Kwiatkowski, and N. Pott, 1998, "Next-toleading order matching for the magnetic photon penguin operator in the $B \rightarrow X_{s} \gamma$ decay," Nucl. Phys. B 517, 353 .

Buras, A. J., and M. Munz, 1995, "Effective Hamiltonian for $B \rightarrow X_{s} e^{+} e^{-}$beyond leading logarithms in the NDR and HV schemes," Phys. Rev. D 52, 186.

Buras, A. J., A. Romanino, and L. Silvestrini, 1998, " $K$ $\rightarrow \pi \nu \bar{\nu}$ : A model-independent analysis and supersymmetry," Nucl. Phys. B 520, 3.

Buras, A. J., and L. Silvestrini, 1999, "Upper bounds on $K$ $\rightarrow \pi \nu \bar{\nu}$ and $K_{L} \rightarrow \pi^{0} e^{+} e^{-}$from $\epsilon^{\prime} / \epsilon$ and $K_{L} \rightarrow \mu^{+} \mu^{-}$," Nucl. Phys. B 546, 299.

Carena, M., D. Garcia, U. Nierste, and C. E. Wagner, 2001, " $b \rightarrow s \gamma$ and supersymmetry with large $\tan (\beta)$," Phys. Lett. B 499, 141.

Cella, G., G. Curci, G. Ricciardi, and A. Vicere, 1994, "QCD corrections to electroweak processes in an unconventional scheme: Application to the $b \rightarrow s \gamma$ decay," Nucl. Phys. B 431, 417.

Chamseddine, A. H., R. Arnowitt, and P. Nath, 1982, "Locally supersymmetric grand unification," Phys. Rev. Lett. 49, 970. Chankowski, P. H., and L. Slawianowska, 2001, " $B_{d, s}^{0} \rightarrow \mu^{-} \mu^{+}$ decay in the MSSM," Phys. Rev. D 63, 054012.

Chay, J., H. Georgi, and B. Grinstein, 1990, "Lepton energy distributions in heavy-meson decays from QCD," Phys. Lett. B 247, 399.

Chen, J. W., G. Rupak, and M. J. Savage, 1997, "Non-1/m $m_{b}^{n}$ power suppressed contributions to inclusive $B \rightarrow X_{s} \ell^{+} \ell^{-}$decays," Phys. Lett. B 410, 285.

Chen, S., et al., (CLEO Collaboration), 2001, "Branching fraction and photon energy spectrum for $b \rightarrow s \gamma$," Phys. Rev. Lett. 87, 251807.

Chetyrkin, K., M. Misiak, and M. Munz, 1997, "Weak radiative $B$-meson decay beyond leading logarithms," Phys. Lett. B 400, 206.

Chetyrkin, K., M. Misiak, and M. Munz, 1998a, "Beta functions and anomalous dimensions up to three loops," Nucl. Phys. B 518, 473.
Chetyrkin, K., M. Misiak, and M. Munz, 1998b, “ $|\Delta(F)|=1$ nonleptonic effective Hamiltonian in a simpler scheme," Nucl. Phys. B 520, 279.

Cho, P. L., M. Misiak, and D. Wyler, 1996, " $K_{L} \rightarrow \pi^{0} e^{+} e^{-}$and $B \rightarrow X_{s} \ell^{+} \ell^{-}$decay in the MSSM," Phys. Rev. D 54, 3329.

Choudhury, S. R., and N. Gaur, 1999, "Dileptonic decay of $B_{s}$ meson in SUSY models with large $\tan (\beta)$," Phys. Lett. B 451, 86.

Chua, C., X. He, and W. Hou, 1999, " $C P$-violating $b \rightarrow s \gamma$ decay in supersymmetric models," Phys. Rev. D 60, 014003.

Chun, E. J., K. Hwang, and J. S. Lee, 2000, " $C P$ asymmetries in radiative $B$ decays with $R$-parity violation," Phys. Rev. D 62, 076006.

Ciuchini, M., G. Degrassi, P. Gambino, and G. F. Giudice, 1998a, "Next-to-leading QCD corrections to $B \rightarrow X_{s} \gamma$ : Standard model and two Higgs doublet model," Nucl. Phys. B 527, 21.

Ciuchini, M., G. Degrassi, P. Gambino, and G. F. Giudice, 1998b, "Next-to-leading QCD corrections to $B \rightarrow X_{s} \gamma$ in supersymmetry," Nucl. Phys. B 534, 3.

Ciuchini, M., E. Franco, G. Martinelli, M. Pierini, and L. Silvestrini, 2001, "Charming penguins strike back," Phys. Lett. B 515, 33.

Ciuchini, M., E. Franco, G. Martinelli, and L. Reina, 1994, "The $\Delta S=1$ effective Hamiltonian including next-to-leading order QCD and QED corrections," Nucl. Phys. B 415, 403.

Ciuchini, M., E. Franco, G. Martinelli, L. Reina, and L. Silvestrini, 1993, "Scheme independence of the effective Hamiltonian for $b \rightarrow s \gamma$ and $b \rightarrow s g$ decays," Phys. Lett. B 316, 127. CLEO Collaboration, 2003, http://www.lns.cornell.edu/public/ CLEO/

Coan, T. E., et al., (CLEO Collaboration), 2000, "Study of exclusive radiative $B$-meson decays," Phys. Rev. Lett. 84, 5283. Coan, T. E., et al., (CLEO Collaboration), 2001, “CP asymmetry in $b \rightarrow s \gamma$ decays," Phys. Rev. Lett. 86, 5661.

Cohen, A. G., D. B. Kaplan, and A. E. Nelson, 1996, “The more minimal supersymmetric standard model," Phys. Lett. B 388, 588.

Colangelo, G., and G. Isidori, 1998, "Supersymmetric contributions to rare kaon decays: Beyond the single mass-insertion approximation," J. High Energy Phys. 09, 009.

Colangelo, G., and G. Isidori, 2001, "An introduction to CHPT," hep-ph/0101264.

Colangelo, P., and A. Khodjamirian, 2000, "QCD sum rules: A modern perspective," hep-ph/0010175.

Cooper, P. S., (CKM Collaboration), 2001, “CKM: Charged kaons at the main injector," Nucl. Phys. B (Proc. Suppl.) 99A, 121; see also http://www.fnal.gov/projects/ckm/Welcome.html Czarnecki, A., and W. J. Marciano, 1998, "Electroweak radiative corrections to $b \rightarrow s \gamma$," Phys. Rev. Lett. 81, 277.

D'Ambrosio, G., G. F. Giudice, G. Isidori, and A. Strumia, 2002, "Minimal flavour violation: An effective field theory approach," Nucl. Phys. B 645, 155.

D'Ambrosio, G., and G. Isidori, 2002, “ $K^{+} \rightarrow \pi^{+} \nu \bar{\nu}$ : A rising star on the stage of flavour physics," Phys. Lett. B 530, 108. de Boer, W., M. Huber, A. V. Gladyshev, and D. I. Kazakov, 2001, "The $b \rightarrow X_{s} \gamma$ rate and Higgs boson limits in the constrained minimal supersymmetric model," Eur. Phys. J. C 20, 689.

Dedes, A., H. K. Dreiner, and U. Nierste, 2001, "Correlation of $B_{s} \rightarrow \mu^{+} \mu^{-}$and $(g-2)_{\mu}$ in minimal supergravity," Phys. Rev. Lett. 87, 251804. 
Dedes, A., and A. Pilaftsis, 2003, "Resummed effective Lagrangian for Higgs-mediated FCNC interactions in the CP-violating MSSM,” Phys. Rev. D 67, 015012.

Degrassi, G., P. Gambino, and G. F. Giudice, 2000, “ $B \rightarrow X_{s} \gamma$ in supersymmetry: Large contributions beyond the leading order," J. High Energy Phys. 12, 009.

Demir, D. A., and K. A. Olive, 2002, " $B \rightarrow X_{s} \gamma$ in supersymmetry with explicit $C P$ violation," Phys. Rev. D 65, 034007.

de Rafael, E., 2001, "Large-N(c) QCD and low energy interactions," in QCD Theory and Experiment, AIP Conference Proceedings No. 602, edited by P. Colangelo and G. Nordulli (AIP, Melville, NY), p. 14.

de Rafael, E., 2002, “The muon g-2 revisited," hep-ph/0208251. Deshpande, N. G., X. He, and J. Trampetic, 1996, "Longdistance contributions to penguin processes $b \rightarrow s \gamma$ and $b$ $\rightarrow d \gamma$," Phys. Lett. B 367, 362 .

Deshpande, N. G., J. Trampetic, and K. Panose, 1989, "Resonance background to the decays $b \rightarrow s \ell^{+} \ell^{-}, B \rightarrow K^{*} \ell^{+} \ell^{-}$, and $B \rightarrow K \ell^{+} \ell^{-}$," Phys. Rev. D 39, 1461.

Dimopoulos, S., and G. F. Giudice, 1995, "Naturalness constraints in supersymmetric theories with nonuniversal soft terms," Phys. Lett. B 357, 573.

Dimopoulos, S., and S. Raby, 1981, "Supercolor," Nucl. Phys. B 192, 353.

Dine, M., W. Fischler, and M. Srednicki, 1981, "Supersymmetric technicolor," Nucl. Phys. B 189, 575.

Dine, M., R. Leigh, and A. Kagan, 1993, "Flavor symmetries and the problem of squark degeneracy," Phys. Rev. D 48, 4269.

Dine, M., and A. E. Nelson, 1993, "Dynamical supersymmetry breaking at low energies," Phys. Rev. D 48, 1277.

Dine, M., A. E. Nelson, Y. Nir, and Y. Shirman, 1996, "New tools for low-energy dynamical supersymmetry breaking," Phys. Rev. D 53, 2658.

Dine, M., A. E. Nelson, and Y. Shirman, 1995, "Low-energy dynamical supersymmetry breaking simplified," Phys. Rev. D 51, 1362.

Donoghue, J. F., H. P. Nilles, and D. Wyler, 1983, "Flavor changes in locally supersymmetric theories," Phys. Lett. B 128, 55 .

Dugan, M. J., and B. Grinstein, 1991, "QCD basis for factorization in decays of heavy mesons," Phys. Lett. B 255, 583.

E949 Collaboration, 2003, http://www.phy.bnl.gov/e949/

Eidemuller, M., and M. Jamin, 2001, "Charm quark mass from QCD sum rules for the charmonium system," Phys. Lett. B 498, 203.

Ellis, J. R., M. K. Gaillard, D. V. Nanopoulos, and S. Rudaz, 1977, "The phenomenology of the next left-handed quarks," Nucl. Phys. B 131, 285; 132, 541(E).

Everett, L., G. L. Kane, S. Rigolin, L. T. Wang, and T. T. Wang, 2002, "Alternative approach to $b \rightarrow s \gamma$ in the uMSSM," J. High Energy Phys. 201, 022.

Falk, A. F., A. Lewandowski, and A. A. Petrov, 2001, "Effects from the charm scale in $K^{+} \rightarrow \pi^{+} \nu \bar{\nu}$," Phys. Lett. B 505, 107.

Falk, A. F., M. Luke, and M. J. Savage, 1994, "Nonperturbative contributions to the inclusive rare decays $B \rightarrow X_{s} \gamma$ and $B \rightarrow X_{s} \ell^{+} \ell^{-}$," Phys. Rev. D 49, 3367.

Ferrara, S., and E. Remiddi, 1974, "Absence of the anomalous magnetic moment in a supersymmetric Abelian gauge theory," Phys. Lett. B 53, 347.

Fleischer, R., 1999, "New strategies to extract $\beta$ and $\gamma$ from $B_{d} \rightarrow \pi^{+} \pi^{-}$and $B_{s} \rightarrow K^{+} K^{-}$," Phys. Lett. B 459, 306.
Gabbiani, F., E. Gabrielli, A. Masiero, and L. Silvestrini, 1996, "A complete analysis of FCNC and CP constraints in general SUSY extensions of the standard model," Nucl. Phys. B 477, 321.

Gambino, P., M. Gorbahn, and U. Haisch, 2003, hep-ph/0306079.

Gambino, P., and U. Haisch, 2000, "Electroweak effects in radiative $B$ decays," J. High Energy Phys. 9, 001.

Gambino, P., and U. Haisch, 2001, "Complete electroweak matching for radiative $B$ decays," J. High Energy Phys. 110, 020 .

Gambino, P., and M. Misiak, 2001, "Quark mass effects in $\bar{B}$ $\rightarrow X_{s} \gamma$," Nucl. Phys. B 611, 338.

Gasser, J., 2000, "Chiral perturbation theory," Nucl. Phys. B, Proc. Suppl. 86, 257.

Ghinculov, A., T. Hurth, G. Isidori, and Y. P. Yao, 2003a, "Forward-backward asymmetry in $B \rightarrow X_{s} \ell^{+} \ell^{-}$at the NNLL level,” Nucl. Phys. B 648, 254.

Ghinculov, A., T. Hurth, G. Isidori, and Y. P. Yao, 2003b, "NNLL QCD corrections to the decay $B \rightarrow X_{s} \ell^{+} \ell^{-}$," Nucl. Phys. B, Proc. Suppl. 116, 284.

Ghinculov, A., T. Hurth, G. Isidori, and Y. P. Yao, 2003c, "The decay $B \rightarrow X_{s} \ell^{+} \ell^{-}$at the NNLL level: Dilepton mass spectrum," CERN-TH/2003-132.

Giudice, G. F., M. A. Luty, H. Murayama, and R. Rattazzi, 1998, "Gaugino mass without singlets," J. High Energy Phys. 12, 027.

Giusti, L., A. Romanino, and A. Strumia, 1999, "Natural ranges of supersymmetric signals," Nucl. Phys. B 550, 3.

Golutvin, A., 2001, "Heavy flavor physics," in Proceedings of the XXXth International Conference on High Energy Physics (ICHEP 2000), Osaka, Japan, July 2000, edited by C. S. Lih and T. Yamenaka (World Scientific, Singapore), p. 61.

Goto, T., Y. Y. Keum, T. Nihei, Y. Okada, and Y. Shimizu, 1999, "Effect of supersymmetric $C P$ phases on the $B \rightarrow X_{s} \gamma$ and $B \rightarrow X_{s} \ell^{+} \ell^{-}$decays in the minimal supergravity model," Phys. Lett. B 460, 333.

Goto, T., Y. Okada, and Y. Shimizu, 1998, "Flavor-changing neutral-current processes in $B$ and $K$ decays in the supergravity model,” Phys. Rev. D 58, 094006.

Goto, T., Y. Okada, Y. Shimizu, and M. Tanaka, 1997, "b $\rightarrow s \ell \bar{\ell}$ in the minimal supergravity model," Phys. Rev. D 55, 4273; 66, 019901(E).

Grant, A. K., A. G. Morgan, S. Nussinov, and R. D. Peccei, 1997, "Comment on nonperturbative $O\left(1 / m_{c}^{2}\right)$ corrections to $\Gamma\left(\bar{B} \rightarrow X_{s} \gamma\right)$," Phys. Rev. D 56, 3151 .

Greub, C., and T. Hurth, 1996, "Towards a next-to-leading logarithmic result in $B \rightarrow X_{s} \gamma$," hep-ph/9608449.

Greub, C., and T. Hurth, 1997, "Two-loop matching of the dipole operators for $b \rightarrow s \gamma$ and $b \rightarrow s$ gluon," Phys. Rev. D 56, 2934.

Greub, C., and T. Hurth, 1999, "Radiative corrections in inclusive rare B decays," Nucl. Phys. B (Proc. Suppl.) 74A, 247.

Greub, C., T. Hurth, M. Misiak, and D. Wyler, 1996, "The $c$ $\rightarrow u \gamma$ contribution to weak radiative charm decay," Phys. Lett. B 382, 415.

Greub, C., T. Hurth, and M. Steinhauser, 2003, private communication.

Greub, C., T. Hurth, and D. Wyler, 1996a, "Virtual corrections to the decay $b \rightarrow s \gamma$," Phys. Lett. B 380, 385 . 
Greub, C., T. Hurth, and D. Wyler, 1996b, "Virtual $O\left(\alpha_{s}\right)$ corrections to the inclusive decay $b \rightarrow s \gamma$," Phys. Rev. D 54, 3350. Greub, C., and P. Liniger, 2001, "Calculation of next-to-leading QCD corrections to $b \rightarrow s$ gluon," Phys. Rev. D 63, 054025.

Greub, C., H. Simma, and D. Wyler, 1995, "Branching ratio and direct $C P$-violating rate asymmetry of the rare decays $B \rightarrow K^{*} \gamma$ and $B \rightarrow \rho \gamma$," Nucl. Phys. B 434, 39; 444, 447(E).

Grinstein, B., M. J. Savage, and M. B. Wise, 1989, " $B$ $\rightarrow X_{s} e^{+} e^{-}$in the six quark model," Nucl. Phys. B 319, 271.

Grinstein, B., R. P. Springer, and M. B. Wise, 1988, "Effective Hamiltonian for weak radiative $B$-meson decay," Phys. Lett. B 202, 138.

Gronau, M., 2000, "U-spin symmetry in charmless $B$ decays," Phys. Lett. B 492, 297.

Gronau, M., Y. Grossman, D. Pirjol, and A. Ryd, 2002, "Measuring the photon helicity in radiative $B$ decays," Phys. Rev. Lett. 88, 051802.

Gronau, M., and D. Pirjol, 2002, "Photon polarization in radiative $B$ decays," Phys. Rev. D 66, 054008.

Gronau, M., and J. L. Rosner, 2001, "U-spin symmetry in doubly Cabibbo-suppressed charmed meson decays," Phys. Lett. B 500, 247.

Groom, D. E., et al. (Particle Data Group Collaboration), 2000, "Review of particle physics," Eur. Phys. J. C 15, 1.

Grossman, Y., and Y. Nir, 1997, " $K_{L} \rightarrow \pi^{0} \nu \bar{\nu}$ beyond the standard model," Phys. Lett. B 398, 163.

Grossman, Y., and D. Pirjol, 2000, "Extracting and using photon polarization information in radiative $B$ decays," J. High Energy Phys. 06, 029.

Grossman, Y., Z. Ligeti, and E. Nardi, 1996, "First limit on inclusive $B \rightarrow X_{s} \nu \bar{\nu}$ decay and constraints on new physics," Nucl. Phys. B 465, 369; 480, 753(E).

Hagelin, J. S., S. Kelley, and T. Tanaka, 1994, "Supersymmetric flavor changing neutral currents: Exact amplitudes and phenomenological analysis," Nucl. Phys. B 415, 293.

Hagiwara, K., et al., (Particle Data Group Collaboration), 2002, "Review of particle physics," Phys. Rev. D 66, 010001. Hall, L., V. A. Kostelecky, and S. Raby, 1986, "New flavor violations in supergravity models," Nucl. Phys. B 267, 415.

Hall, L., J. Lykken, and S. Weinberg, 1983, "Supergravity as the messenger of supersymmetry breaking," Phys. Rev. D 27, 2359.

Hamzaoui, C., M. Pospelov, and M. Toharia, 1999, "Higgsmediated FCNC in supersymmetric models with large $\tan (\beta)$," Phys. Rev. D 59, 095005.

Hewett, J. L., 1996, “ $\tau$ polarization asymmetry in $B$ $\rightarrow X_{s} \tau^{+} \tau^{-}$," Phys. Rev. D 53, 4964.

Hewett, J. L., and J. D. Wells, 1997, "Searching for supersymmetry in rare $B$ decays," Phys. Rev. D 55, 5549.

Hiller, G., and A. Kagan, 2002, "Probing for new physics in polarized $\Lambda_{b}$ decays at the $Z$," Phys. Rev. D 65, 074038.

Huang, C. S., W. Liao, and Q. S. Yan, 1999, “The promising process to distinguish super-symmetric models with large $\tan (\beta)$ from the standard model: $B \rightarrow X_{s} \mu^{+} \mu^{-}$," Phys. Rev. D 59, 011701.

Huang, C. S., W. Liao, Q. S. Yan, and S. H. Zhu, 2001, " $B_{s}$ $\rightarrow \ell^{+} \ell^{-}$in a general 2HDM and MSSM," Phys. Rev. D 63, 114021; 64, 059902(E).

Hurth, T., 2001, "Inclusive rare B decays," hep-ph/0106050.
Hurth, T., and T. Mannel, 2001a, " $C P$ asymmetries in $b$ $\rightarrow(s / d)$ transitions as a test of CKM CP violation," Phys. Lett. B 511, 196.

Hurth, T., and T. Mannel, 2001b, "Direct $C P$ violation in radiative $B$ decays," in $Q C D$ Theory and Experiment, AIP Conference Proceedings No. 602, edited by P. Colangelo and G. Nardulli (AIP, Melville, NY), p. 212.

Hurth, T., et al., 2001, "Present and future CP measurements," J. Phys. G 27, 1277.

Isgur, N., and M. B. Wise, 1989, "Weak decays of heavy mesons in the static quark approximation," Phys. Lett. B 232, 113.

Isgur, N., and M. B. Wise, 1990, "Weak transition form factors between heavy mesons," Phys. Lett. B 237, 527.

Isidori, G., and A. Retico, 2001, "Scalar flavour-changing neutral currents in the large $\tan (\beta)$ limit," J. High Energy Phys. 11, 001.

Jessop, C., 2002, “A world average for $B \rightarrow X_{s} \gamma$," SLAC-PUB9610.

Kagan, A. L., and M. Neubert, 1998, "Direct $C P$ violation in $B \rightarrow X_{s} \gamma$ decays as a signature of new physics," Phys. Rev. D 58, 094012.

Kagan, A. L., and M. Neubert, 1999, “QCD anatomy of $B$ $\rightarrow X_{s} \gamma$ decays," Eur. Phys. J. C 7, 5 .

Kagan, A. L., and J. Rathsman, 1997, "Hints for enhanced $b$ $\rightarrow$ s gluon from charm and kaon counting," hep-ph/9701300.

Kaneko, J., et al., (BELLE Collaboration), 2003, "Measurement of the electroweak penguin process $B \rightarrow X_{s} \ell^{+} \ell^{-}$," Phys. Rev. Lett. 90, 021801.

Keum, Y. Y., H. N. Li, and A. I. Sanda, 2001, "Penguin enhancement and $B \rightarrow K \pi$ decays in perturbative QCD," Phys. Rev. D 63, 054008.

Keum, Y. Y., and A. I. Sanda, 2003, "Possible large direct $C P$ violations in charmless $B$ decays," Phys. Rev. D 67, 054009.

Khodjamirian, A., and R. Ruckl, 1998, "QCD sum rules for exclusive decays of heavy mesons," hep-ph/9801443.

Khodjamirian, A., R. Ruckl, G. Stoll, and D. Wyler, 1997, "QCD estimate of the long-distance effect in $B \rightarrow K^{*} \gamma$," Phys. Lett. B 402, 167.

Khodjamirian, A., G. Stoll, and D. Wyler, 1995, "Calculation of long-distance effects in exclusive weak radiative decays of $B$ mesons," Phys. Lett. B 358, 129.

Kiers, K., A. Soni, and G. Wu, 2000, "Direct $C P$ violation in radiative $b$ decays in and beyond the standard model," Phys. Rev. D 62, 116004.

Kim, C. S., Y. G. Kim, C. D. Lu, and T. Morozumi, 2000, “Azimuthal angle distribution in $B \rightarrow K^{*}(\rightarrow K \pi) \ell^{+} \ell^{-}$at low invariant $m_{\ell_{+} \ell_{-}}$region," Phys. Rev. D 62, 034013.

Kim, Y. G., P. Ko, and J. S. Lee, 1999, "Possible new physics signals in $b \rightarrow s \gamma$ and $b \rightarrow s \ell^{+} \ell^{-}$," Nucl. Phys. B 544, 64 .

Kobayashi, M., and T. Maskawa, 1973, "CP violation in the renormalizable theory of weak interaction," Prog. Theor. Phys. 49, 652.

Kruger, F., and L. M. Sehgal, 1996, "Lepton polarization in the decays $B \rightarrow X_{s} \mu^{+} \mu^{-}$and $B \rightarrow X_{s} \tau^{+} \tau^{-}$," Phys. Lett. B 380, 199.

Kruger, F., L. M. Sehgal, N. Sinha, and R. Sinha, 2000, “Angular distribution and $C P$ asymmetries in the decays $\bar{B}$ $\rightarrow K^{-} \pi^{+} e^{-} e^{+}$and $\bar{B} \rightarrow \pi^{-} \pi^{+} e^{-} e^{+}, "$ Phys. Rev. D 61, 114028; 63, 019901(E). 
Kudenko, Y. G., 2001, "KOPIO experiment at BNL," in Kaon Decay Physics, edited by S. Sugimoto and T. Yamanaka (KEK, Tsukuba), KEK Proceedings-2001-23, p. 331.

Kuhn, J. H., and M. Steinhauser, 2001, "Determination of $\alpha_{s}$ and heavy quark masses from recent measurements of $R(s)$," Nucl. Phys. B 619, 588; 640, 415(E).

Leibovich, A. K., Z. Ligeti, and M. B. Wise, 2002, "Enhanced subleading structure functions in semileptonic $B$ decay," Phys. Lett. B 539, 242.

Lellouch, L., 2001, "Light hadron weak matrix elements," Nucl. Phys. B (Proc. Suppl.) 94A, 142.

Lellouch, L., 2002, "Phenomenology from lattice QCD," hep-ph/0211359.

Lellouch, L., and M. Lüscher, 2001, "Weak transition matrix elements from finite-volume correlation functions," Commun. Math. Phys. 219, 31.

Lenz, A., U. Nierste, and G. Ostermaier, 1999, "Determination of the CKM angle $\gamma$ and $|V(u b) / V(c b)|$ from inclusive direct $C P$ asymmetries and branching ratios in charmless $B$ decays," Phys. Rev. D 59, 034008.

Leurer, M., Y. Nir, and N. Seiberg, 1994, "Mass matrix models: The Sequel," Nucl. Phys. B 420, 468.

Ligeti, Z., M. E. Luke, A. V. Manohar, and M. B. Wise, 1999, "The $\bar{B} \rightarrow X_{s} \gamma$ photon spectrum," Phys. Rev. D 60, 034019 .

Ligeti, Z., L. Randall, and M. B. Wise, 1997, "Comment on nonperturbative effects in $\bar{B} \rightarrow X_{s} \gamma$," Phys. Lett. B 402, 178.

Lim, C. S., T. Morozumi, and A. I. Sanda, 1989, "A prediction for $d \Gamma(b \rightarrow s \ell \bar{\ell}) / d q^{2}$ including the long-distance effects," Phys. Lett. B 218, 343.

Lingel, K., T. Skwarnicki, and J. G. Smith, 1998, "Penguin decays of $B$ mesons," Annu. Rev. Nucl. Part. Sci. 48, 253.

Lunghi, E., A. Masiero, I. Scimemi, and L. Silvestrini, 2000, " $B \rightarrow X_{s} \ell^{+} \ell^{-}$decays in super-symmetry," Nucl. Phys. B 568, 120.

Mannel, T., and M. Neubert, 1994, "Resummation of nonperturbative corrections to the lepton spectrum in inclusive $B$ $\rightarrow X \ell \bar{\nu}$ decays," Phys. Rev. D 50, 2037.

Mannel, T., and S. Recksiegel, 1997, "Probing the helicity structure of $b \rightarrow s \gamma$ in $\Lambda_{b} \rightarrow \Lambda \gamma$," Acta Phys. Pol. B 28, 2489. Manohar, A. V., 1998, "Large N QCD," hep-ph/9802419.

Manohar, A. V., and M. B. Wise, 1994, "Inclusive semileptonic $B$ and polarized $\Lambda_{b}$ decays from QCD," Phys. Rev. D 49, 1310.

Manohar, A. V., and M. B. Wise, 2000, "Heavy Quark Physics," Cambridge Monogr. Part. Phys., Nucl. Phys., Cosmol. 10, 1.

Martin, A. D., J. Outhwaite, and M. G. Ryskin, 2001, "Improving $\alpha_{e m}\left(M_{Z}^{2}\right)$ and the charm mass by analytic continuation," Eur. Phys. J. C 19, 681.

Masiero, A., and O. Vives, 2001, "New physics in $C P$-violation experiments," Annu. Rev. Nucl. Part. Sci. 51, 161.

Melikhov, D., N. Nikitin, and S. Simula, 1998, "Probing righthanded currents in $B \rightarrow K^{*} \ell^{+} \ell^{-}$transitions," Phys. Lett. B 442, 381.

Misiak, M., 1993, "The $b \rightarrow s e^{+} e^{-}$and $b \rightarrow s \gamma$ decays with nextto-leading logarithmic QCD corrections," Nucl. Phys. B 393, 23; 439, 461(E).

Misiak, M., 2000a, "Theory of radiative $B$ decays," hep-ph/0002007.

Misiak, M., 2000b, "Status of theoretical $\bar{B} \rightarrow X_{s} \gamma$ and $B$ $\rightarrow X_{s} \ell^{+} \ell^{-}$analyses," hep-ph/0009033.
Misiak, M., 2000c, "Radiative $B$ decay in the SM," hep-ph/0105312.

Misiak, M., 2002, private communication.

Misiak, M., and J. Urban, 1999, "QCD corrections to FCNC decays mediated by $Z$ penguins and $W$ boxes," Phys. Lett. B 451, 161.

Narison, S., 2001, “ $c, b$ quark masses and $f\left(D_{s}\right), f\left(B_{s}\right)$ decay constants from pseudoscalar sum rules in full QCD to order $\alpha_{s}^{2}$," Phys. Lett. B 520, 115.

Neubert, M., 1994a, "Analysis of the photon spectrum in inclusive $B \rightarrow X_{s} \gamma$ decays," Phys. Rev. D 49, 4623.

Neubert, M., 1994b, "Heavy quark symmetry," Phys. Rep. 245, 259.

Neubert, M., 2000, "On the inclusive determination of $|V(u b)|$ from the lepton invariant mass spectrum," J. High Energy Phys. 07, 022.

Nir, Y., and N. Seiberg, 1993, "Should squarks be degenerate?," Phys. Lett. B 309, 337.

Nir, Y., and M. P. Worah, 1998, "Probing the flavor and $C P$ structure of supersymmetric models with $K \rightarrow \pi \nu \bar{\nu}$ decays," Phys. Lett. B 423, 319.

Okumura, K. i., and L. Roszkowski, 2002, "Deconstraining supersymmetry from $b \rightarrow s \gamma$," hep-ph/0208101.

Penarrocha, J., and K. Schilcher, 2001, "QCD duality and the mass of the charm quark," Phys. Lett. B 515, 291.

Politzer, H. D., and M. B. Wise, 1991, "Perturbative corrections to factorization in $\bar{B}$ decay," Phys. Lett. B 257, 399.

Pomarol, A., and D. Tommasini, 1996, "Horizontal symmetries for the supersymmetric flavour problem," Nucl. Phys. B 466, 3.

Pott, N., 1996, "Bremsstrahlung corrections to the decay $b$ $\rightarrow s \gamma$," Phys. Rev. D 54, 938.

Randall, L., and R. Sundrum, 1999, "Out of this world supersymmetry breaking,” Nucl. Phys. B 557, 79.

Ricciardi, G., 1995, "Short- and long-distance interplay in inclusive $B \rightarrow X_{d} \gamma$ decays," Phys. Lett. B 355, 313.

Rolf, J., and S. Sint, 2002, "A precise determination of the charm quark's mass in quenched QCD," J. High Energy Phys. 212, 007.

Sachrajda, C. T., 2001, "Lattice B physics," Nucl. Instrum. Methods Phys. Res. A 462, 23.

Shifman, M. A., 1998, "Snapshots of hadrons or the story of how the vacuum medium determines the properties of the classical mesons which are produced, live, and die in the QCD vacuum,” Prog. Theor. Phys. Suppl. 131, 1.

Shifman, M. A., 2000, "Quark-hadron duality," hep-ph/0009131.

Shifman, M. A., A. I. Vainshtein, and V. I. Zakharov, 1978, "On the weak radiative decays (effects of strong interactions at short distances)," Phys. Rev. D 18, 2583; 19, 2815(E).

Soares, J. M., 1991, “CP violation in radiative $b$ decays," Nucl. Phys. B 367, 575.

Stone, S., 2001, “B phenomenology,” hep-ph/0112008.

Strumia, A., 1998, "Two-loop heavy top corrections to the $b$ $\rightarrow s \gamma$ decay," Nucl. Phys. B 532, 28.

Thorndike, E. H., (CLEO Collaboration), 2002, "Radiative $B$ decays: An experimental overview," hep-ex/0206067.

Ushiroda, Y., (BELLE Collaboration), 2001, "Radiative $B$-meson decay," hep-ex/0104045.

Voloshin, M. B., 1997, "Large $O\left(m_{c}^{-2}\right)$ nonperturbative correction to the inclusive rate of the decay $B \rightarrow X_{s} \gamma$," Phys. Lett. B 397, 275. 
Wang, J. c., (BTeV Collaboration), 2002, "The BTeV experiment at the Tevatron collider," hep-ex/0207009.

Xiong, Z., and J. M. Yang, 2002, "Rare $B$-meson dileptonic decays in minimal supersymmetric model," Nucl. Phys. B 628, 193. 